\title{
Tc99m/T1201 cross-talk corrections on a dedicated cardiac CZT SPECT camera
}

\author{
by
}

Stéphanie Chiasson

A thesis submitted to the Faculty of Graduate and Postdoctoral Affairs in partial fulfillment of the requirements for the degree of

\author{
Master of Science \\ in
}

Physics

Ottawa-Carleton Institute for Physics

Department of Physics

Carleton University

Ottawa, Ontario

(C) 2013, Stéphanie Chiasson 
Library and Archives

Canada

Published Heritage

Branch

395 Wellington Street

Ottawa ON K1A ON4

Canada
Bibliothèque et

Archives Canada

Direction du

Patrimoine de l'édition

395 , rue Wellington

Ottawa ON K1A ON4

Canada
Your file Votre référence

ISBN: 978-0-494-94628-2

Our file Notre référence

ISBN: $978-0-494-94628-2$
NOTICE:

The author has granted a nonexclusive license allowing Library and Archives Canada to reproduce, publish, archive, preserve, conserve, communicate to the public by telecommunication or on the Internet, loan, distrbute and sell theses worldwide, for commercial or noncommercial purposes, in microform, paper, electronic and/or any other formats.

The author retains copyright ownership and moral rights in this thesis. Neither the thesis nor substantial extracts from it may be printed or otherwise reproduced without the author's permission.
AVIS:

L'auteur a accordé une licence non exclusive permettant à la Bibliothèque et Archives Canada de reproduire, publier, archiver, sauvegarder, conserver, transmettre au public par télécommunication ou par l'Internet, prêter, distribuer et vendre des thèses partout dans le monde, à des fins commerciales ou autres, sur support microforme, papier, électronique et/ou autres formats.

L'auteur conserve la propriété du droit d'auteur et des droits moraux qui protege cette thèse. $\mathrm{Ni}$ la thèse ni des extraits substantiels de celle-ci ne doivent être imprimés ou autrement reproduits sans son autorisation.
In compliance with the Canadian Privacy Act some supporting forms may have been removed from this thesis.

While these forms may be included in the document page count, their removal does not represent any loss of content from the thesis.
Conformément à la loi canadienne sur la protection de la vie privée, quelques formulaires secondaires ont été enlevés de cette thèse.

Bien que ces formulaires aient inclus dans la pagination, il n'y aura aucun contenu manquant. 


\begin{abstract}
Single Photon Emission Computed Tomography (SPECT) is a standard method for evaluating heart disease. A new dedicated cardiac camera with CZT detectors offers improved energy resolution and sensitivity compared to standard SPECT systems.

Simultaneous $\mathrm{Tc} 99 \mathrm{~m} / \mathrm{T} 1201$ protocols are fast, but correction for cross-talk between isotopes is necessary to achieve good image quality. The Triple-Energy-Window (TEW) correction method is easy to implement and provides accurate scatter estimation in single-isotope studies.

We retrospectively assessed the cross-talk correction using clinically acquired single-isotope studies: 52 T1201 studies and $52 \mathrm{Tc} 99 \mathrm{~m}$-tetrofosmin studies, matched by gender and BMI. Projection data from Tl-stress and Tc-rest studies were combined to create contaminated data before reconstruction. TEW corrections were evaluated in both primary energy windows. Modifications to the corrections were required. The modified approach results in residual cross-talk as low as $2 \%$ but high noise levels were present in the corrected images. Further modifications are needed to reduce noise.
\end{abstract}




\section{Acknowledgements}

I would like to greatly thank my supervisor R. Glenn Wells, for his tremendous patience and guidance and for giving me the opportunity to work with him these past years. I would also like to thank my colleague Amir Pourmoghaddas for providing image arithmetic software that was needed during my research.

Without the constant support of my family, especially my parents Muriel and Barry, I would not be where I am today. I would like to thank them for all the support they have given me throughout the years, encouraging me to work hard and follow my dreams every step of the way.

Finally, I would like to thank everyone at the University of Ottawa Heart Institute for their help along the way. It has been a great experience, collaborating with such great people. 


\section{Table of Contents}

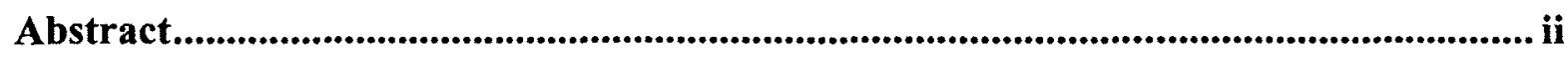

Acknowledgements ...................................................................................................................... iii

Table of Contents ........................................................................................................................ v

List of Tables ................................................................................................................... ix

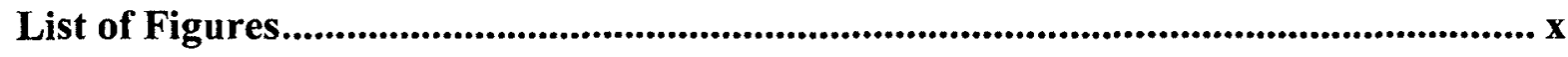

List of Acronyms ............................................................................................................................ xii

1 Chapter: Introduction .......................................................................................... 1

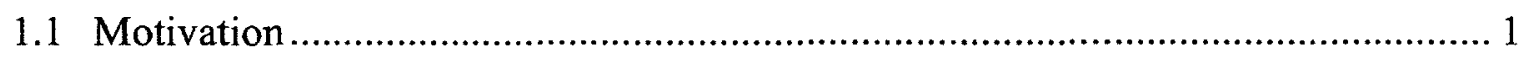

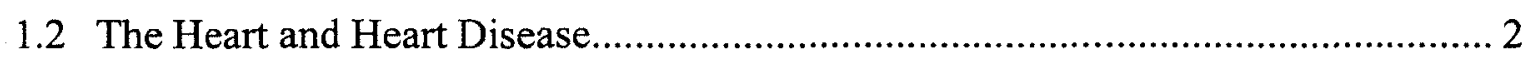

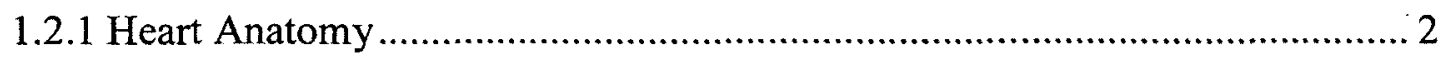

1.2.1.1 Heart Imaging - Axes...................................................................... 3

1.2.2 Ischemic Heart Disease ....................................................................... 3

1.3 Radioactivity and Interactions with Matter......................................................... 4

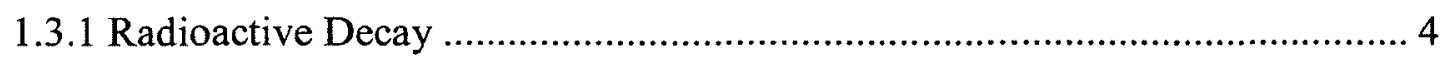

1.3.2 Interactions of Radiation with Matter .................................................... 5

1.3.2.1 Photoelectric Effect........................................................................ 5

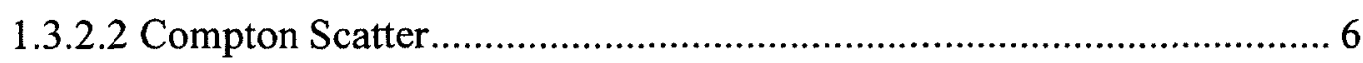

1.4 Single Photon Emission Computed Tomography ................................................. 8

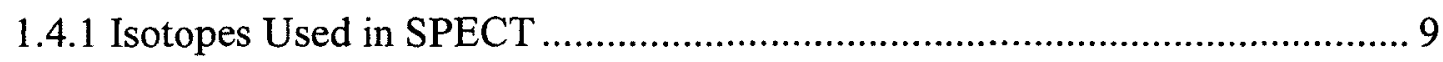

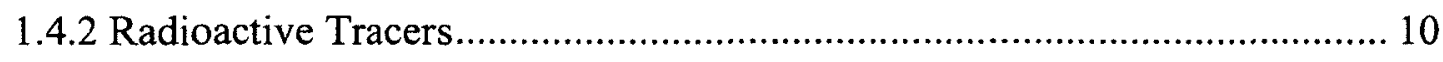

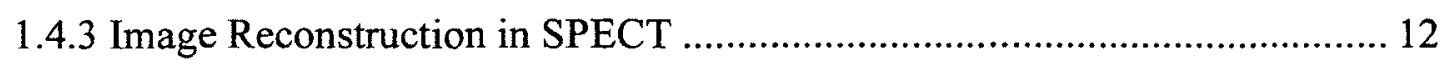


1.4.3.1 Maximum Likelihood Expectation Maximization Reconstruction.... 12

1.5 Cameras Used in SPECT ................................................................... 13

1.5.1 Anger Scintillation Camera ............................................................. 15

1.5.1.1 Parallel-hole Collimator ........................................................ 15

1.5.1.2 Scintillation Detectors and Photomultiplier Tubes ..................... 17

1.5.2 CZT Camera

1.5.2.1 Pinhole Collimator ............................................................. 20

1.5.2.2 Solid State Detectors ....................................................... 22

1.5.3 Differences Between Scintillation and Solid State Detectors .................... 23

1.5.3.1 Low Energy Tail of the CZT Detector.................................... 26

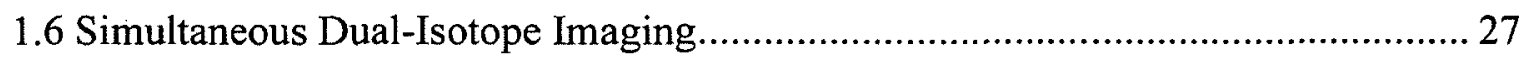

1.6.1 Complications in Simultaneous Dual-Isotope Imaging ........................ 30

1.6.1.1 High Effective Dose to Patient............................................. 31

1.6.1.2 Presence of Cross-talk Between Isotopes .................................. 32

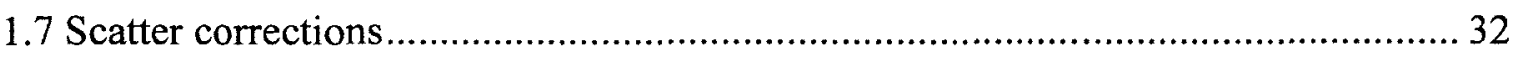

1.7.1 Scatter Corrections Based on Modeling the Scatter Distribution ................ 34

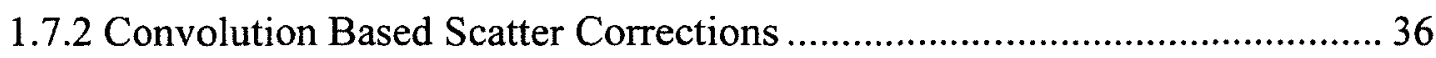

1.7.3 Energy Based Scatter Corrections............................................... 37

1.7.3.1 Triple Energy Window Scatter Correction Method ....................... 38

1.7.4 Cross-talk Correction Methods in Dual-Isotope Imaging ....................... 39

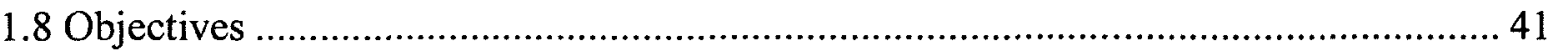


2 Chapter: Cross-talk Correction with a Dedicated Cardiac Camera ..................... 44

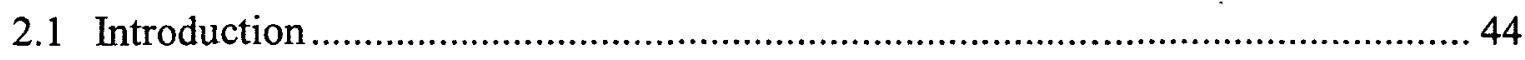

2.1.1 Motivation for Dual-Isotope Imaging ........................................................ 44

2.1.2 Complications in Multi-isotope Imaging ..................................................4 45

2.1.3 CZT Camera

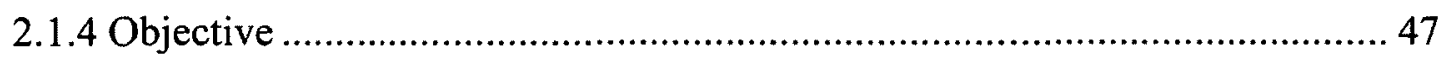

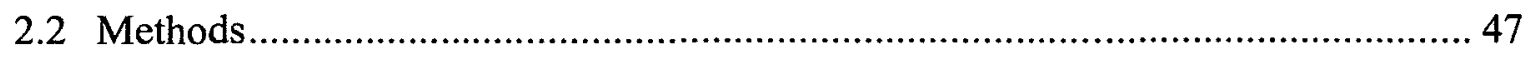

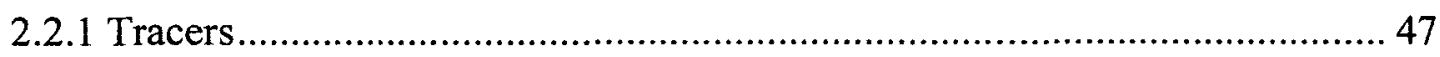

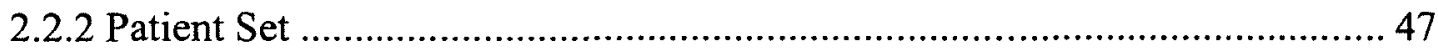

2.2.3 Cross-talk Compensation - Triple Energy Window Method ........................ 49

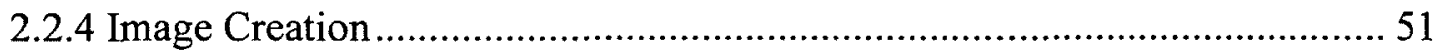

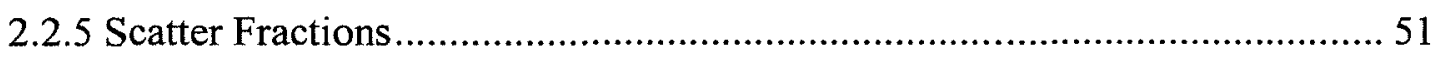

2.2.6 Phase 1 - Estimation of T1201 Cross-talk in the Tc99m Primary Window . 52

2.2.6.1 Energy Windows Considered....................................................... 52

2.2.6.2 Modifications to TEW ………….................................................... 53

2.2.7 Phase 2 - Estimation of Tc99m Cross-talk in the T1201 Primary Window . 55

2.2.7.1 Energy Windows Considered............................................................. 56

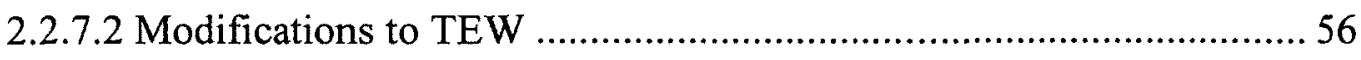

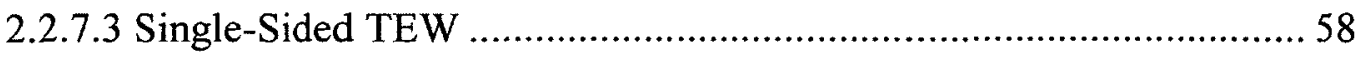

2.2.8 Phase 3 - Simultaneous Dual-Isotope Corrections.......................................... 61

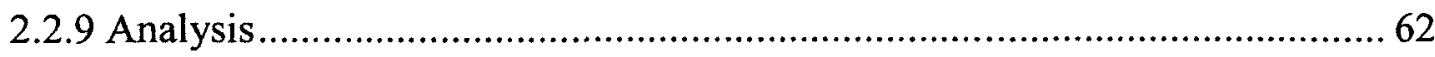

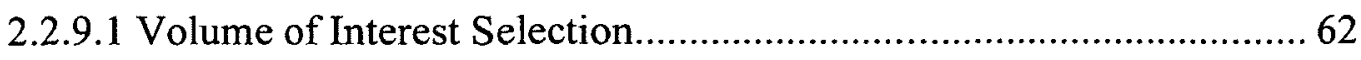

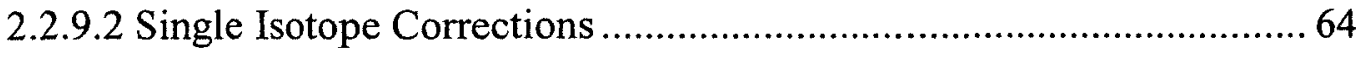




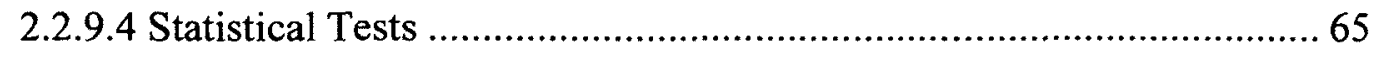

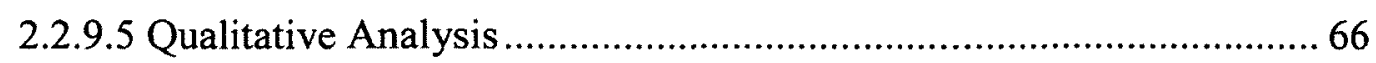

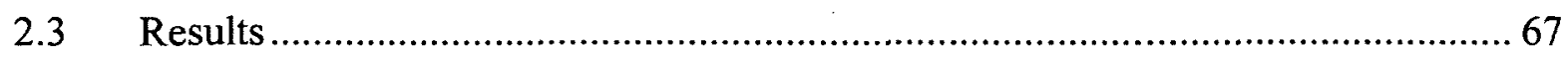

2.3.1 Phase 1 - Estimation of T1201 Cross-talk in the Tc99m Window ................ 67

2.3.1.1 Dependence of Scaling Factor on BMI............................................. 73

2.3.2 Phase 2 - Estimation of Tc99m Cross-talk in the T1201 Primary Window . 74

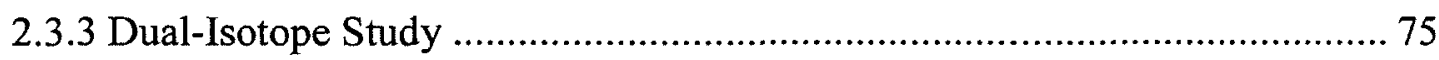

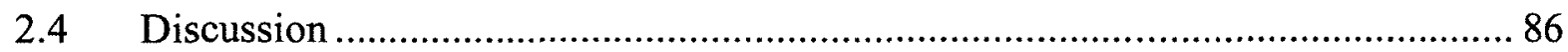

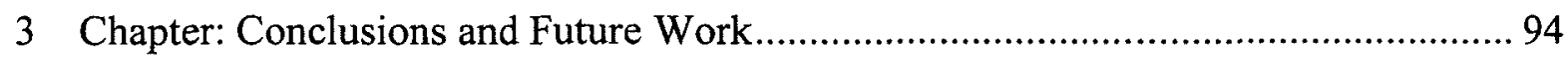

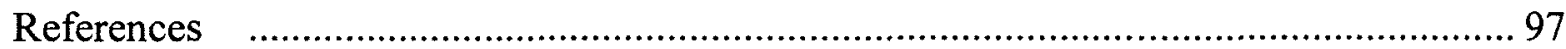




\section{List of Tables}

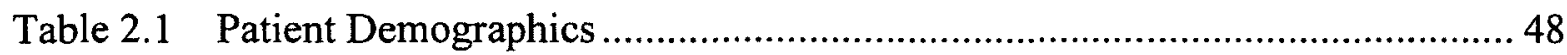

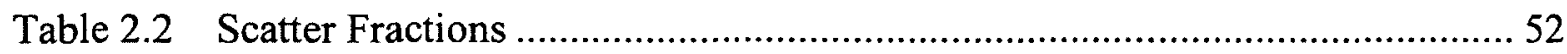

Table 2.3 Energy Window Information for Corrections in Tc99m Primary Window.... 54

Table 2.4 Energy Window Information for Corrections in T1201 Primary Window ..... 58

Table 2.5 Energy Window Information for Single-Sided Corrections in T1201 Primary

Window

Table 2.6 Scaling Factors of $167 \mathrm{keV}$ Peak in Tc99m Window Corrections ................ 72 


\section{List of Figures}

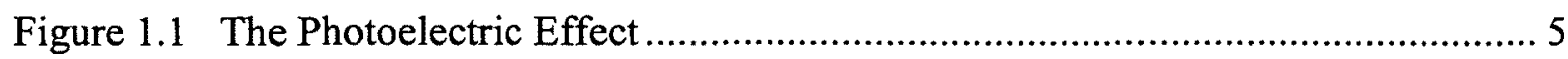

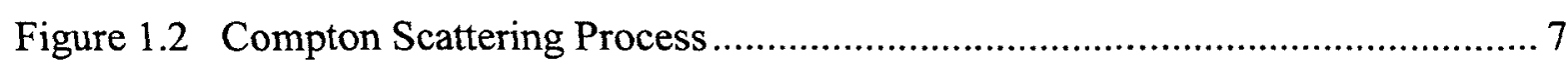

Figure 1.3 Itterative Reconstruction Process ………................................................ 14

Figure 1.4 Principal Components of a Typical Anger Camera...................................... 16

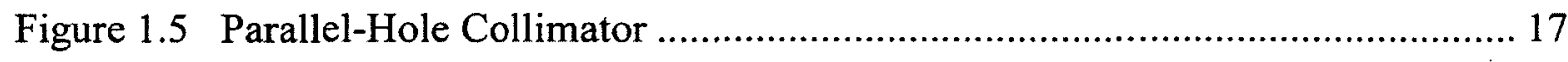

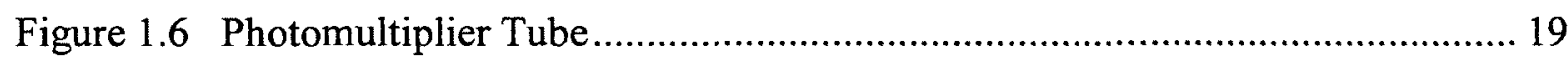

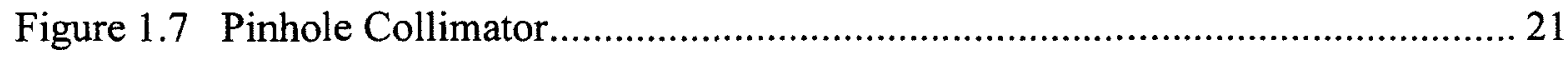

Figure 1.8 Discovery NM 530c from GE Healthcare Camera....................................... 24

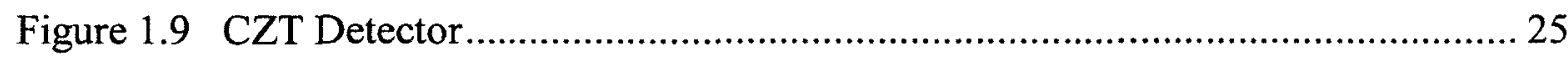

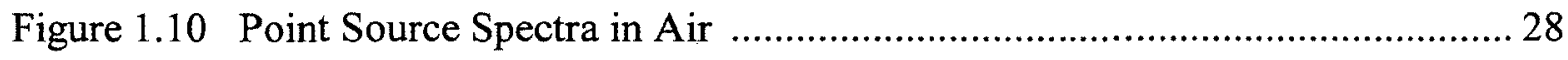

Figure 1.11 1-Day Single-Isotope Tc99m Image Acquisition Protocol ......................... 29

Figure 1.12 Simultaneous Acquisition Dual-Isotope Imaging Protocol ........................ 30

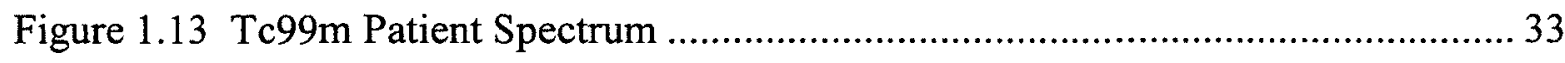

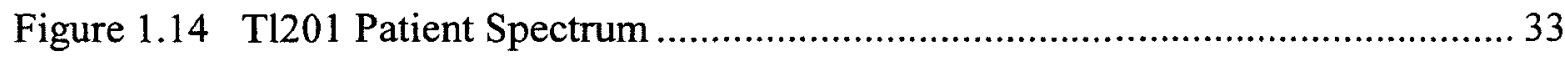

Figure 1.15 Triple Energy Window Scatter Correction............................................... 40

Figure 1.16 Triple Energy Window Scatter Correction in Dual-Isotope Imaging ..........42

Figure 2.1 Single Sided Triple Energy Window Scatter Correction ...............................60

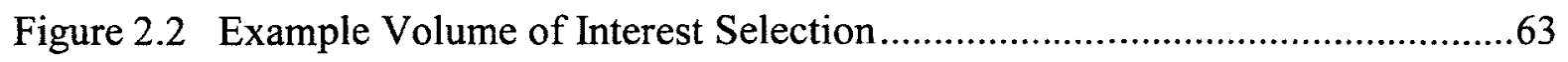

Figure 2.3 17-Segment Heart Model for Qualitative Analysis of Corrections ................66

Figure 2.4 Mean vs. Standard Deviation of Pixels in Corrected Image of T1201 Cross- 
talk in Tc99m Window

Figure 2.5 Mean vs. Standard Deviation of Pixels in Corrected Image of T1201 Crosstalk in Tc99m Window with Added Scaled Subtraction .70

Figure 2.6 Mean vs. Standard Deviation of Pixels in Corrected Image of T1201 Crosstalk in Tc99m Window with Added Scaled Subtraction (Rescaled) .71

Figure 2.7 Ideal Scaling Factor vs. BMI .73

Figure 2.8 Mean vs. Standard Deviation of Pixels in Corrected Image of Tc99m Crosstalk in T1201 Window .76

Figure 2.9 Mean Difference vs. Standard Deviation in Corrected Dual-Isotope Image in Tc99m Window 78

Figure 2.10 Example Heart Images and Polar Maps in Tc99m Window .80

Figure 2.11 Mean Difference vs. Standard Deviation in Corrected Dual-Isotope Image in T1201 Window .82

Figure 2.12 Example Heart Images and Polar Maps in Tl201 Window .83

Figure 2.13 Mean vs. Standard Deviation of Pixels in Corrected Image of Tc99m CrossTalk in T1201 Window (Single Sided Correction) .85

Figure 2.14 Mean Difference vs. Standard Deviation in Corrected Dual-Isotope Image in T1201 Window (Single Sided Correction) .87

Figure 2.15 Example Heart Images and Polar Maps in T1201 Window (with Single Sided Correction) .88 


\section{List of Acronyms}

Ant. Anterior wall

ASNC American Society of Nuclear Cardiology

BMI. Body Mass Index

CT Computed Tomography

CZT. Cadmium Zinc Telluride

EC Electron Capture

ESSE . Effective Scatter Source Estimation

FADS Factor Analysis of Dynamic Structures

FOV. Field of View

FWHM Full Width at Half Maximum

HLA Horizontal Long Axis

IHD Ischemic Heart Disease

Inf... Inferior wall

IT. Isomeric Transition

Lat. Lateral wall 
MLEM Maximum Likelihood Expectation Maximization

MPI Myocardial Perfusion Imaging

NM Nuclear Medicine

PMT Photomultiplier Tube

SA Short Axis

$\mathrm{SD}$ Standard Deviation

Sep. Septum

SPECT. Single Photon Emission Computed Tomography

SRS Summed Rest Scores

SSS Summed Stress Scores

TEW Triple Energy Window

UOHI. University of Ottawa Heart Institute

VLA Vertical Long Axis

VOI Volume of Interest 


\section{Chapter 1: Introduction}

\subsection{Motivation}

Heart disease remains one of the main causes of death in Canada. In 2008, heart disease accounted for $29 \%$ of all deaths in Canada. Furthermore, $90 \%$ of Canadians have a minimum of one risk factor for heart disease, bringing the need for more efficient and accurate methods for diagnosing heart disease. Of all cardiovascular deaths in $2008,54 \%$ of these were due to Ischemic Heart Disease. (1)

Single Photon Emission Computed Tomography (SPECT) is one of the standard methods used to clinically evaluate heart disease and the severity of defects in the heart. The typical diagnostic protocols for myocardial perfusion imaging that are currently implemented in the clinic use only one isotope for both the rest and stress states of the heart. This leads to a total time of approximately four hours for a same-day rest-stress 
Tc99m acquisition, due to the wait needed between acquisitions to minimize interference of the tracer from the first injection in the second image. (2)

SPECT offers the possibility of performing simultaneous dual-isotope imaging (38), as multiple isotopes can be used for the same study. Proposed protocols in can reduce the total time to 30 minutes as well as having many more advantages. However, the presence of interference between the isotopes presents a major problem and must be accounted for before simultaneous dual-isotope SPECT can be clinically implemented. $(9-12)$.

\subsection{The Heart and Heart Disease}

\subsubsection{Heart Anatomy}

The heart is the organ whose main responsibility is to pump oxygen and nutrient rich blood throughout the whole body to other vital organs. It must have a constant flow of blood carrying plenty of oxygen and other nutrients in order to have the energy it requires to function properly. It is composed of four chambers. The two upper chambers are called the right and left atria, while the two lower ones are the right and left ventricles. Walls of muscle called the septa separate the left and right atria and the left and right ventricles. (13) 


\subsubsection{Heart Imaging - Axes}

Images of the heart will be viewed as a cross section of the heart in different orientations. The three main axes used in this study are the Horizontal long axis (HLA), Vertical long axis (VLA) and Short axis (SA).

A good way of visualizing the heart axes is to picture the heart as a cylinder that is closed at one end. The axis of rotation of the cylinder is the long axis of the heart. The SA is perpendicular to the long axis. The HLA and VLA are parallel to the long axis and orthogonal to each other. The HLA cuts horizontally across the heart and shows both the right and left ventricles. The VLA cuts vertically and shows the anterior and inferior walls of the left-ventricle. (14)

\subsubsection{Ischemic heart disease}

Ischemic Heart Disease (IHD), or myocardial ischemia is characterised by a reduced blood flow to the heart. This is typically due to a narrowing of a coronary artery, which supplies blood to the heart. IHD can typically present with an array of symptoms, including but not limited to, angina (chest pains), decreased exercise tolerance and difficulty in breathing or swelling of the extremities due to weakness of the heart muscle.

When the heart is under a stressed condition, which can be achieved through exercise or by administering a drug to the patient, diseased heart muscle will typically have a lower blood flow than its healthy counterpart. By comparing the blood flow in the 
heart muscles when the heart is in a stressed state and a rest state, it is possible to determine how severely the muscle is damaged and guide what further actions should be taken.

\subsection{Radioactivity and interactions with matter}

\subsubsection{Radioactive decay}

Radioactive decay is a process in which an unstable nucleus transforms into a more stable form, emitting particles and releasing energy in the process. The unstable nucleus is referred to as the parent nucleus and the result of the decay is referred to as the daughter nucleus. There are many different types of radioactive decay, including alpha decay, beta decay, electron capture (EC), gamma decay and isomeric transition (IT). The isotopes used in this study decay by IT and EC. (16)

Isomeric transitions deal with a daughter nucleus from a previous decay that is in a long-lived, or metastable, state. Unlike typical gamma decay, where the excited nucleus returns to its ground state in a very short period of time, emitting a photon in the process, the daughter will stay in the excited state for a prolonged amount of time and will not immediately decay to its more stable state. Just as in gamma decay, there will be a photon emitted in an isomeric transition. It only differs from gamma decay in that there is a delay in the time that the reaction happens. (16)

Electron capture occurs when an orbital electron is captured by the nucleus. This decay is represented in equation 1-1. The electron $(e)$ combines with a proton $\left(p^{+}\right)$to 
form a neutron $(n)$. This reaction leaves the atom in an excited state. Characteristic $\mathrm{x}$-rays and Auger electrons may be emitted as the atom returns to a more stable state. Some of the energy emitted is carried away by the emitted neutrino (v). (16)

$$
p^{+}+e^{-} \rightarrow n+v+\text { energy }
$$

\subsubsection{Interactions of Radiation with Matter}

\subsubsection{Photoelectric Effect}

The photoelectric effect is an interaction between photons and matter where an atom absorbs the total energy of an incident photon. The energy absorbed by the atom results in an orbital electron being ejected from the atom. This electron is called a photoelectron. (17)

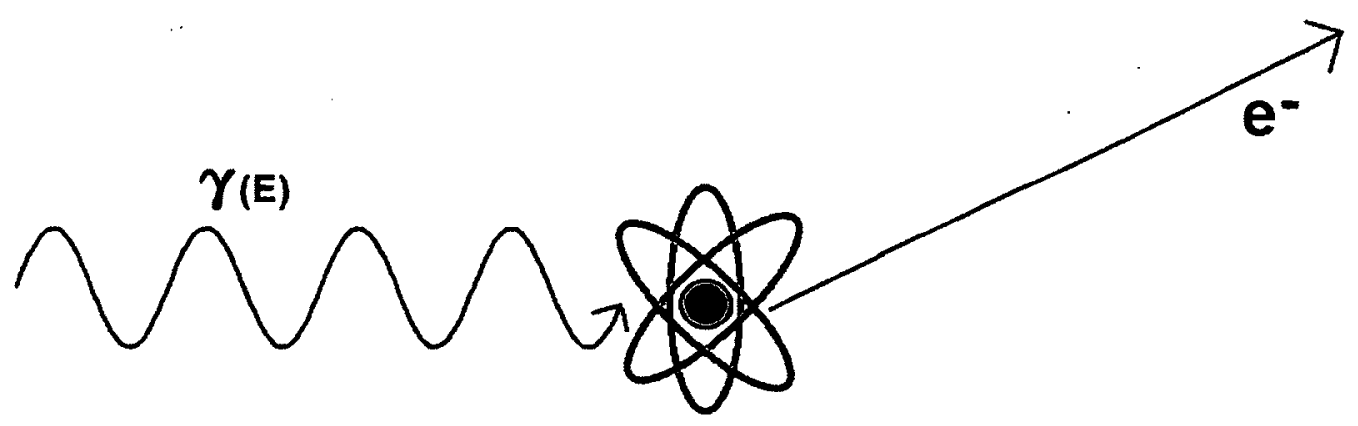

Figure 1.1 The photoelectric effect. A photon $(\gamma)$ of energy E interacts with an atom, resulting in an ejected electron (e-).

In order for this interaction to occur, the incident photon must be of sufficient energy to exceed the binding energy of the electron it will eject. After the interaction, the atom will be left with a vacancy in an orbital electron shell, which will be filled by 
transition of another orbital electron to this vacancy. This will lead to emission of characteristic X-rays or Auger electrons. However, in materials with low atomic number (such as tissues found in the human body), binding energies and characteristic x-ray energies are only a few $\mathrm{keV}$ or less and need not to be considered in diagnostic imaging, as the photons emitted will be absorbed by the body.

\subsubsection{Compton Scatter}

Electromagnetic radiation emitted during radioactive decay will interact with matter as it passes through it, transferring some of its energy to the matter. The type of interaction that will occur will greatly depend on the energy of the photon. At energies required for diagnostic imaging, the principal interaction occurring is Compton scatter. In this inelastic scattering process, a photon will interact with a loosely bound outer shell orbital electron, as shown in figure 1.2. (17)

This interaction will cause the photon to change direction as well as losing some of its energy in the process. The energy lost by the photon is directly related to the angle at which the photon is scattered after interaction with the electron. The resulting energy of the photon after the interaction $\left(E^{\prime}\right)$ is given by equation $1-2$, where $E$ is the initial energy of the photon, $m_{o}$ is the mass of an electron, $c$ is the speed of light in vacuum and $\theta$ is the angle representing the change in direction of the photon. (18) 


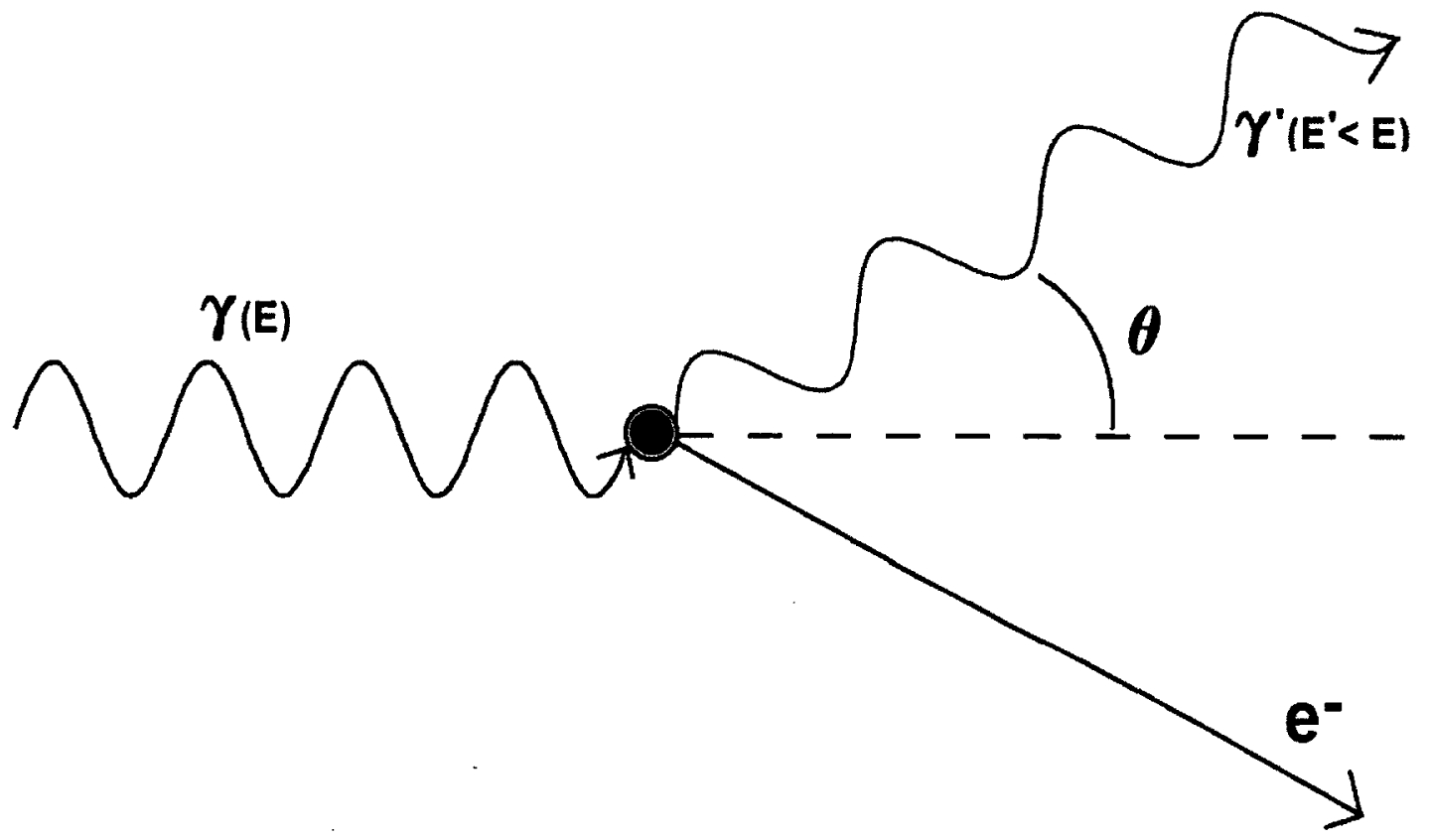

Figure 1.2 Representation of the Compton scattering process. A photon $(\gamma)$ of initial energy $E$ interacts with an electron. Following the interaction, the photon is deflected from its path by a scattering angle $\theta$. The photon has also transferred some of its energy to the now free Compton electron $\left(\mathrm{e}^{-}\right)$, resulting in a new photon energy E', which is less than the initial energy of the photon. 


$$
E^{\prime}=\frac{E}{1+\frac{E}{m_{o} c^{2}}(1-\cos \theta)}
$$

\subsection{Single Photon Emission Computed Tomography}

Single Photon Emission Computed Tomography (SPECT) remains one of the standard techniques for evaluating heart disease. Using this imaging method provides a functional image, as opposed to providing information on anatomy. Functional imaging provides information on a physiological activity within a certain tissue or organ, such as metabolism or blood flow. With SPECT, this is achieved by injecting a compound into the body that has been labeled with a gamma-ray-emitting radionuclide. These compounds are referred to as tracers. The tracers are chosen to behave in a similar way to or react with specific compounds in the body and thus provide information about the behavior of those native compounds. (16)

The imaging method involves acquiring images of the distribution of the activity emitted by the tracer at different angles around the patient. These images are called projection images. From these projections, we can reconstruct a three dimensional image of the distribution of the tracer inside the patient, resulting in an image of the physiological process we are interested in. Each step of this process will be explained in detail in the following sections. 


\subsubsection{Isotopes used in SPECT}

While choosing an isotope suitable for imaging, we need to ensure the half-life of the substance is relatively short. The half-life of a radioactive nuclide is the amount of time required for its activity to decay by half. The longer the half-life, the greater the effective dose that will be delivered to the patient, which is to be kept low for a diagnostic procedure.

However, the half-life of the tracer must be long enough to allow time for the tracer to be prepared, injected into the patient and still allow sufficient time for imaging. (19) The most commonly used isotope for myocardial perfusion imaging (MPI) is Technetium-99m (Tc99m). With a half-life of 6.02 hours, (20) Tc99m allows adequate preparation and imaging times while not delivering too high an effective dose to the patient. The typical amount of activity injected for a one-day Tc99m-MPI study is 370 $\mathrm{MBq}$ at rest and $1100 \mathrm{MBq}$ at stress leading to an effective patient dose of $9.9 \mathrm{mSv}$. (21)

Another commonly used isotope in MPI is Thallium-201, with a half-life of 3.044 days. (22) As the half-life is much longer than Tc99m, the patient radiation effective dose is much higher. To partially compensate for this, less activity is injected. The typical amount injected for a T1201 MPI study is $130 \mathrm{MBq}$ which gives an effective patient dose of $22 \mathrm{mSv} .(21)$

The energy of the photon emitted by the decay is also an important factor in choosing a radioactive tracer for SPECT imaging. Photons are emitted from tracers inside the body and so must travel through patient tissues to reach the camera. As photons pass 
through tissues, they can interact and be attenuated. We must ensure that the energy of these photons is high enough so that they are not greatly attenuated and can escape the body to be detected. However, the energy of the photons has to be low enough to allow interaction with the detector and thus detection of the photons. (16)

Tc99m emits photons at $140 \mathrm{keV}$ with $88.5 \%$ abundance. (20) T1201 emits a range of $\mathrm{Hg} \mathrm{x}$-rays between $68.9-80.3 \mathrm{keV}(94.4 \%)$ as well as gamma emissions at 167 $\mathrm{keV}$ and $135 \mathrm{keV}$ ( $8 \%$ and $2 \%$ respectively). (22) These energies are in the acceptable energy range of $30 \mathrm{keV}$ to $300 \mathrm{keV}(16)$ in terms of minimising attenuation inside the patient while being easily detectable by the typical SPECT gamma cameras.

\subsubsection{Radioactive tracers}

The first step to acquiring an image with SPECT is to inject the subject with a tracer which is a compound that has been labeled with a radioactive nuclide. The compound chosen is different for each metabolic function we wish to image. In the case of myocardial perfusion imaging, we wish to study the function of the heart muscle. The most commonly used tracers for this study are Tc99m-tetrofosmin and Tc99m-sestamibi. The tracer used at the University of Ottawa Heart Institute (UOHI) is Tc $99 \mathrm{~m}$-tetrofosmin and this is the tracer used in this work. It has a very high and rapid uptake in the heart muscle, taking only approximately 5 minutes to reach its maximum uptake and remains static for the length of most imaging procedures. (19) Tc99m-tetrofosmin can be used to detect reversible ischemia and infarcted regions of the heart. Myocardial ischemia is the 
restriction of blood supply to the heart muscle, leading to a lack of oxygen required to keep the tissue alive. Blood flow may be blocked by a blood clot or a slow buildup of a hard substance (plaque) within the arteries of the heart. Blocking the passage of blood prevents oxygen from reaching the heart muscle, causing the cells to die. In some cases, there is adequate blood supply when the heart is in a normal resting state but not when the heart is under a stressed condition with elevated heart rate. When the heart returns to a normal resting heart rate, blood flow returns to normal. This condition is referred to as reversible ischemia. Myocardial infarction is damage to the heart muscle which occurs when blood flow to that part of the heart is blocked for a long period of time leading to death of the heart tissues. (23)

In this study, Tc99m-tetrofosmin will be used to detect changes in perfusion (blood flow) caused by a pharmalogical stress state of the heart (typically induced with the drug dipyridamole). (14)

A second tracer that can be used instead of Tc99m-tetrofosmin for MPI is Tl201. Tl201 is an analogue of potassium. An interesting quality of Tl201 is that it starts redistributing in the heart muscle about 10-15 minutes after injection. This characteristic of T1201 allows for imaging of both the stress and rest states of the heart with a single injection of the tracer, avoiding the two injections that are common for Tc99m-tracer protocols. (14) 


\subsubsection{Image reconstruction in SPECT}

In this study, image reconstruction is done using iterative reconstruction methods. The essence of these methods is the use of an algorithm to approach the true image starting from a very simple estimate (often a blank or uniform image) and to adjust this image until it matches the true image. This is done by calculating projections from each estimate by summing all pixel values along the projection line, which are compared to the true projections acquired. If the projections do not match, the pixel values are updated using the difference between the estimated and actual projections. This process is repeated until there is convergence of the estimated projection data with the acquired projection data. (16) This process is shown in figure 1.3.

\subsubsection{Maximum Likelihood Expectation Maximization Reconstruction}

The particular algorithm supplied by the vendor for reconstruction of data acquired on our dedicated cardiac camera is the Maximum Likelihood Expectation Maximization (MLEM) reconstruction method. MLEM computes the most likely source distribution that would have created the observed projection data with the assumption that the noise in the projection data is Poisson distributed. The method essentially gives greater weight to higher count regions. The reconstruction process is formulated as follows:

$$
\mu_{j}=\sum_{i} P_{i, j} x_{i}
$$


where $\mu_{j}$ is the measured intensity in the $\mathrm{j}^{\text {th }}$ projection element, $P_{i, j}$ is the probability that activity from the $\mathrm{i}^{\text {th }}$ voxel will be detected in the $\mathrm{j}^{\text {th }}$ projection element and $x_{i}$ is the activity level of the $\mathrm{i}^{\text {th }}$ voxel.

The equation used to compute the activity value for the $(\mathrm{k}+1)^{\text {th }}$ iteration is

$$
x_{j}^{k+1}=x_{j}^{k} \frac{\sum_{i}\left(\frac{y_{i}}{\mu_{i}}\right) P_{i j}}{\sum_{i} P_{i j}}
$$

where $x_{j}^{k+1}$ is the new estimate, $x_{j}^{k}$ is the old estimate, $y_{i}$ is the measured projection, $\mu_{i}$ is the calculated projection and $P_{i j}$ is the backprojection. The denominator serves as a normalization element. Convergence of this method tends to be quite slow. $(16,24)$

\subsection{Cameras used in SPECT}

Once the tracer has been injected into the bloodstream, after a few minutes it will have been taken up into the heart muscle. The time for complete uptake will vary depending on which radiotracer is being used. The tracer will emit photons, which can be detected. The most commonly used detector for SPECT imaging is the Anger gamma camera. Recently, novel dedicated cardiac SPECT cameras have been developed based on solid-state detectors. (25-28) 


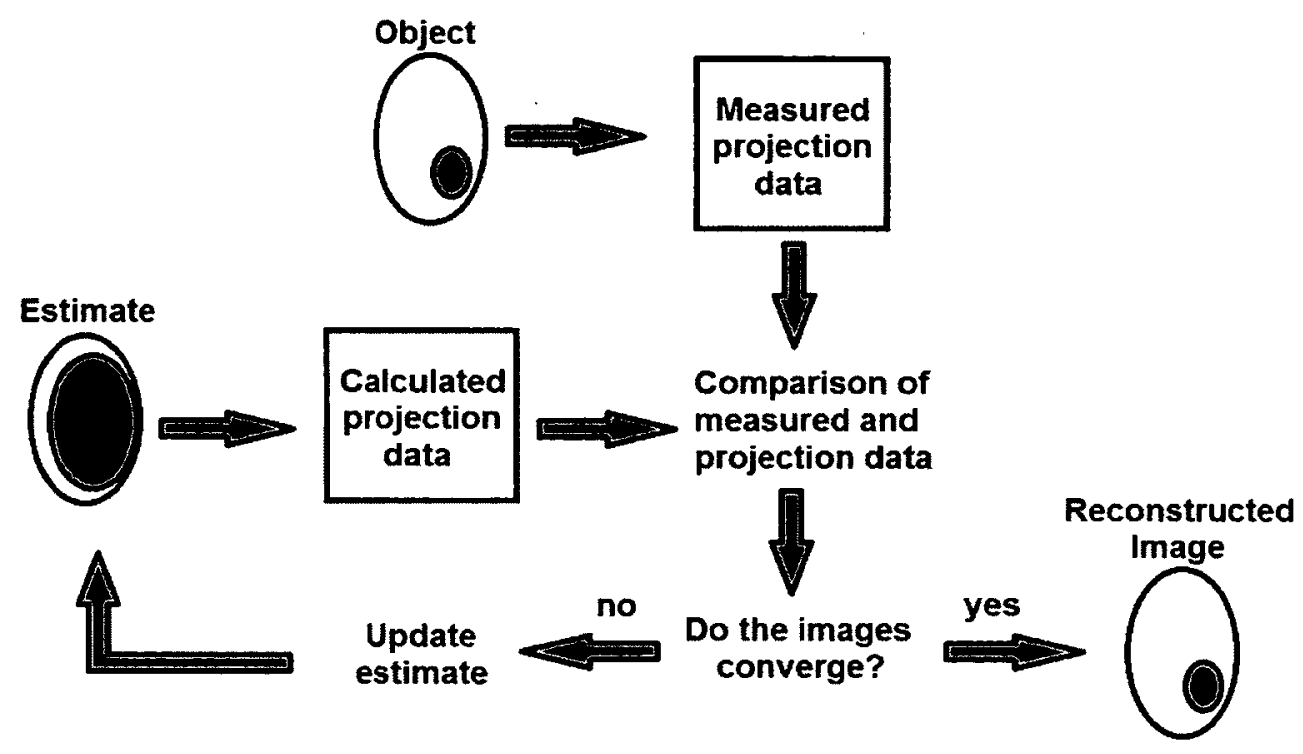

Figure 1.3 Schematic representation of the iterative reconstruction process. An initial image is estimated and projections calculated from the estimate. The calculated projections are compared to the acquired projections. If the projections are not the same, the estimate is adjusted and the process is repeated until convergence is reached. 


\subsubsection{Anger scintillation camera}

The Anger gamma camera remains the standard method of detecting the photons emitted in SPECT imaging. This camera consists of a parallel-hole collimator, a crystal scintillator, typically sodium-iodide (NaI), and an array of photomultiplier tubes (PMT). A representation of a typical Anger camera is shown in figure 1.4.

\subsubsection{Parallel-hole collimator}

In SPECT imaging, a collimator is used prior to the detection of the photons to give directional information on the detected photons. In typical scintillation cameras, parallel hole collimators are widely used. These collimators will allow only photons which are travelling perpendicular to the detector surface to reach the detector. The collimator is made of a material with a high atomic number, typically lead, in order to have high absorbance of photons that are not travelling in the wanted direction. Parallel holes are cast in lead or shaped from lead foils. These collimators project an image onto the detector which is of the same size and orientation as the source distribution. (29) A schematic representation of a typical parallel-hole collimator is shown in figure 1.5 . 


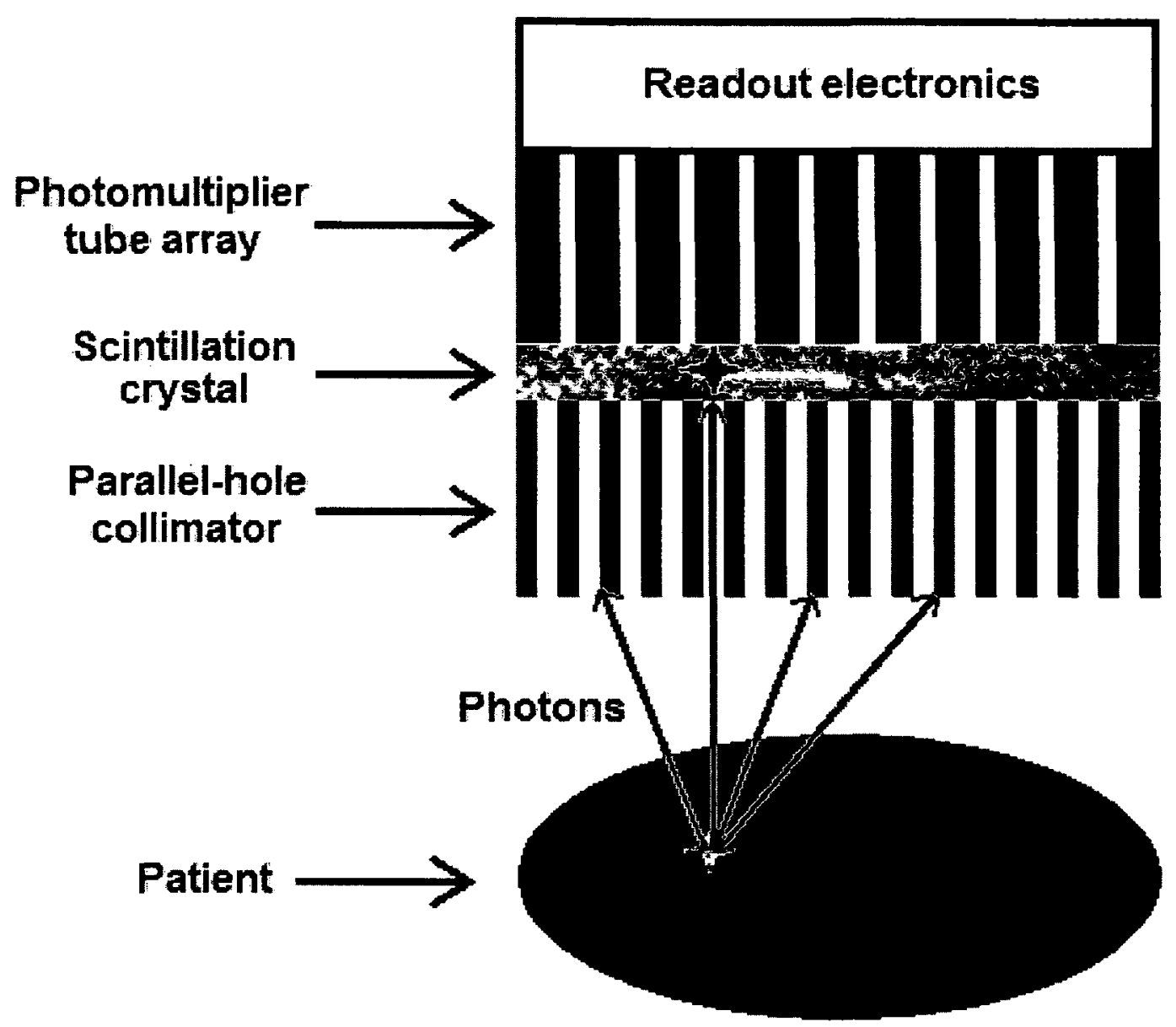

Figure 1.4 Diagram of the principal components of a typical Anger camera. The photons emitted must first pass through a parallel-hole collimator, allowing only photons traveling perpendicular in direction to the crystal to be detected, providing directional information of the detected photons. The photon is then absorbed in the crystal and the corresponding signal is read out and amplified by the photomultiplier tube (PMT) array. The readout electronics determine, record and plot the location of the detected photon. 


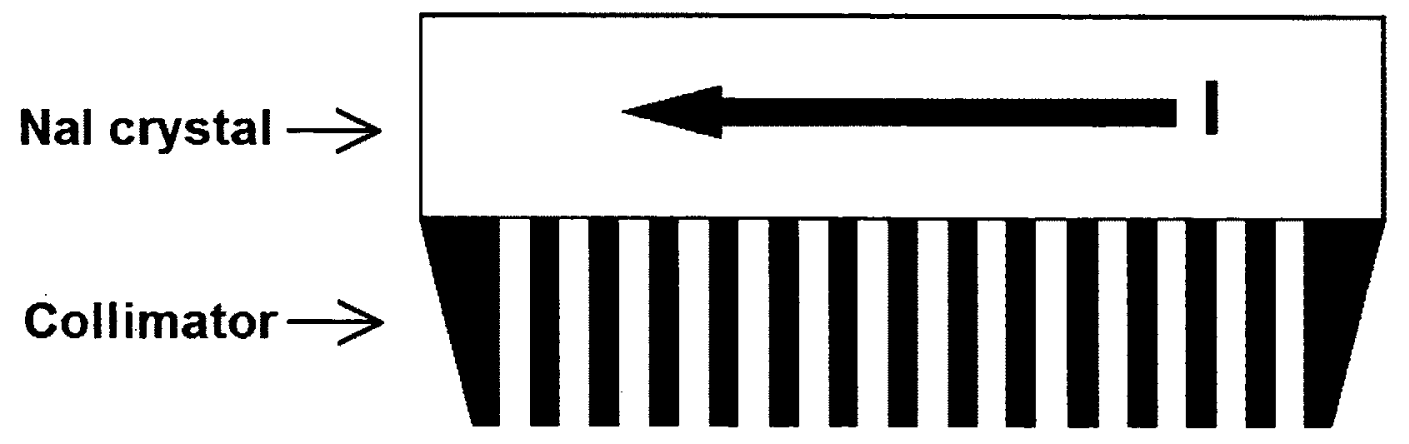

Figure 1.5 Schematic representation of a typical parallel-hole collimator. The image projected onto the detector (I) is the same size and orientation as the source object $(\mathrm{O})$.

\subsubsection{Scintillation detectors and photomultiplier tubes}

Photons from radioactive decay will interact with matter, causing either ionisation and/or excitation of atoms and molecules in the material. When the excited atoms return to their ground state, energy is released. In some materials, the difference in energy between the excited and ground states is a few eV and so this energy is released in the form of visible light. These materials are called scintillators. The typical material used for scintillation detectors in nuclear medicine is sodium-iodide crystal that has been doped with thallium [ $\mathrm{NaI}(\mathrm{Tl})]$. The photons emitted from Tc99m and Tl201 will interact with 
the crystal, and transfer energy to the electrons in the crystal. Many of these electrons will be raised to an excited state. These electrons will then return to their stable state, emitting a light photon in the process. (29)

The light photons are collected by an array of photomultiplier tubes (PMT) attached to the back of the crystal. The light will first be converted to electrons. The PMTs work with very weak light signals, converting them to an electronic signal and amplifying this created signal. The basic principle behind a PMT is illustrated in figure 1.6. The light photon is absorbed at the photocathode and its energy is transferred to a photoelectron which is subsequently ejected. The photoelectron is accelerated to the first dynode by an electrical potential. Multiple photoelectrons will be produced from the interaction of the initial photoelectron with the dynode. The new photoelectrons are then accelerated to the next dynode, where each of these create multiple other photoelectrons. The voltage increases along the tube and the electronic signal gets larger at each dynode. This process creates an electrical signal that is large enough to be shaped and processed once it reaches the anode. (29)

The camera uses an array of PMTs. Each scintillation event produces a shower of light photons. The shower of photons can produce a signal in multiple PMTs. The strength of the summed signal from all of the PMTs is proportional to the energy of the incident gamma ray. A comparison of the relative strengths of the signals from the PMTs can be used to determine the position of the interaction within the crystal. 


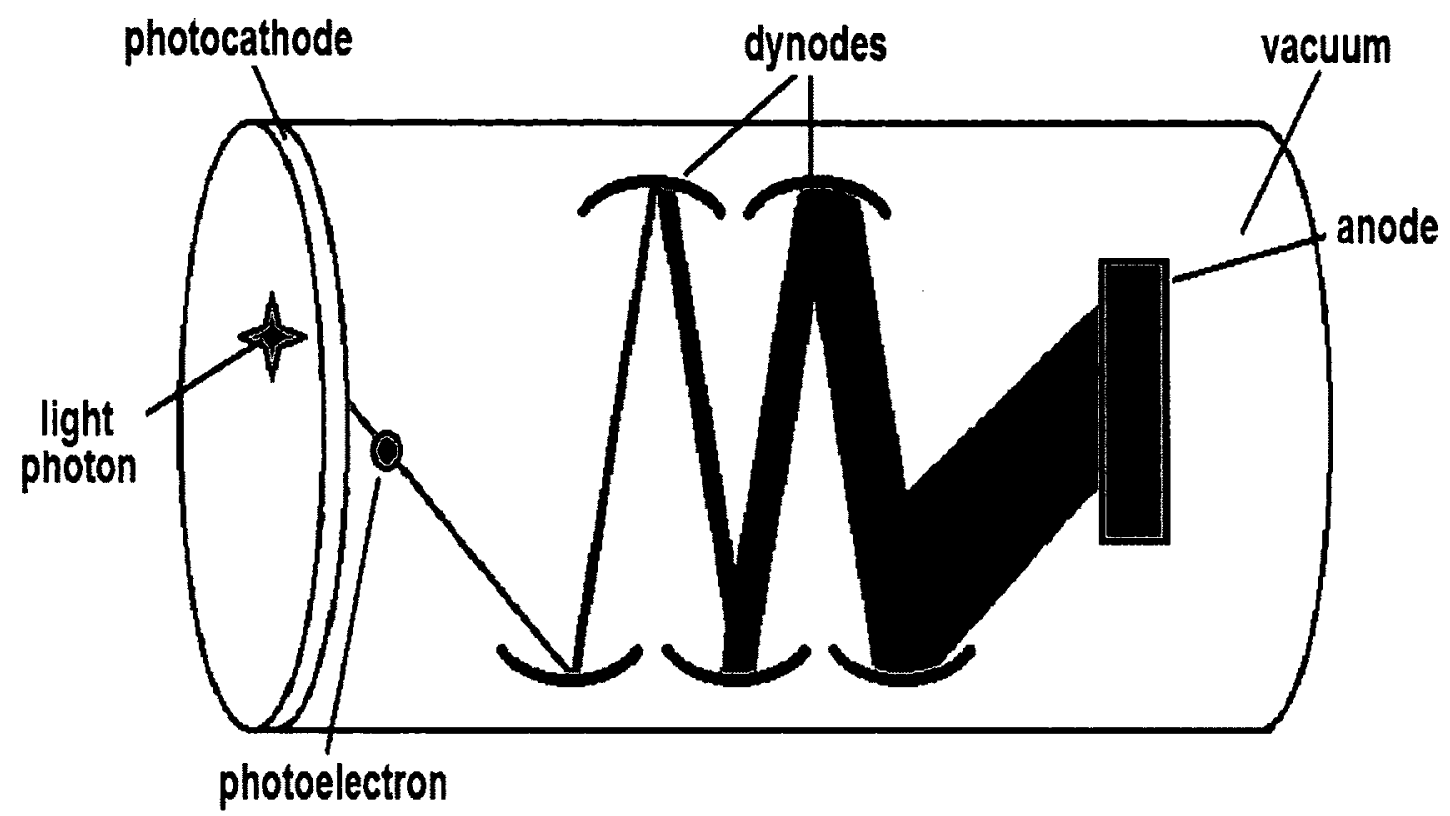

Figure 1.6 Basic principles of a photomultiplier tube. Explanation of principles is provided in text.

\subsubsection{CZT camera}

In recent years, new technologies have been developed to further advance and improve SPECT imaging. (30-33) The camera used in this study is the Discovery NM 530c (GE Healthcare). Unlike the standard SPECT camera, it contains solid-state detectors based on Cadmium-Zinc-Telluride (CZT) instead of scintillation crystals and pinhole collimators replace the typical parallel-hole collimators. The chemical formula for CZT is $\mathrm{Cd}_{1-\mathrm{x}} \mathrm{Zn}_{\mathrm{x}} \mathrm{Te}$, where $\mathrm{x}$ is the fraction of $\mathrm{Zn}$. The value of $\mathrm{x}$ is typically less than 0.2. (34) 
The camera is comprised of a much larger number of detectors (19 instead of 2) but has a much smaller field-of-view $(19 \mathrm{~cm}$ diameter instead of $50 \mathrm{~cm}) .(26)$

\subsubsection{Pinhole collimators}

Pinhole collimators consist of a small pinhole aperture in a slab of material, typically lead, tungsten or any high- $Z$ material with high absorbence of ionizing photons. The pinhole is typically a few millimeters in diameter and is located at the end of a cone. The size of the pinhole can often be varied by using removable inserts. (29)

The principle behind imaging with a pinhole collimator is similar to that of the "box camera". All of the photons from the source that reach the detector must pass through the pinhole. This creates an inverted image of the source on the detector. The pinhole collimator also allows magnification of the image of the source object. Magnification or miniaturization of the image occurs in relation to the ratio of the distance between the object and the collimator and the length of the collimator cone.

$$
\frac{I}{O}=\frac{f}{b}
$$

As indicated in figure 1.7, $\mathrm{I}$ and $\mathrm{O}$ are the sizes of the image and object (source), respectively, while $f$ is the length of the collimator cone and $b$ is the distance between the source and the entrance to the collimator. (16) 


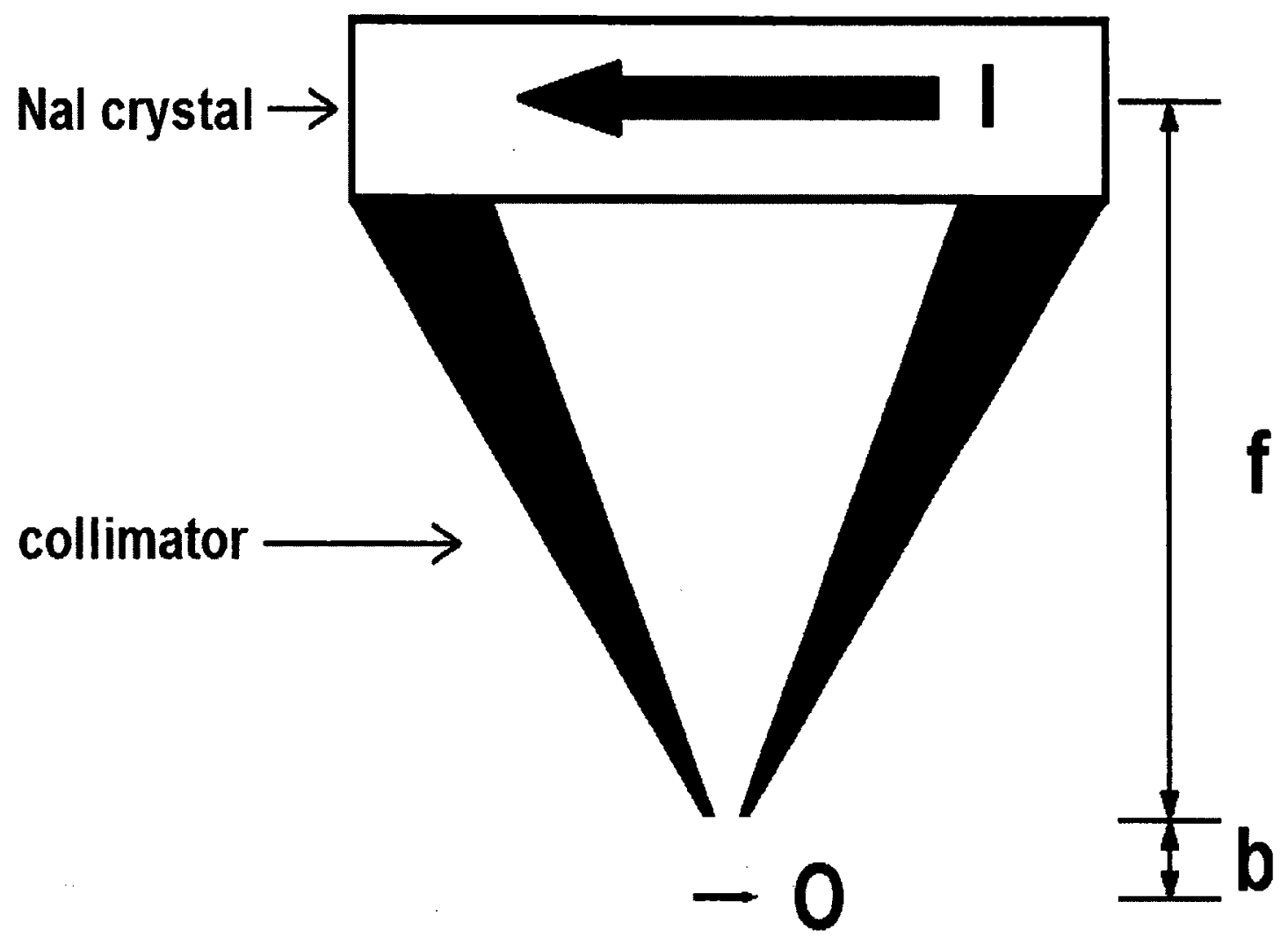

Figure 1.7 Schematic representation of a typical pinhole collimator. The image projected onto the detector (I) is magnified and inverted relative to the source object $(\mathrm{O})$. The magnification depends on the length of the collimator cone (f) as well as the distance between the source and the entrance to the collimator (b). 


\subsubsection{Solid state detectors}

Semiconductor detectors, also known as solid state detectors, are based on crystalline semiconductor materials. Interaction of the materials in a solid state detector with ionizing radiation creates electron-hole pairs in the semiconductor, which are collected by an electric field produced by a reverse voltage. The energy required to create these electron-hole pairs is quite small, therefore for a specific energy, more ionizations occur, providing better energy resolution. (34) Electrodes lie on either side of the semiconductor, collecting the charges produced by the ionizing radiation. $(35,36)$

The intrinsic energy resolution in solid state detectors is better than would be expected from Poisson statistics alone due to correlations in energy loss of the photons in the detector. The energy resolution at a certain energy can be determined by the following equation (35)

$$
F W H M=2.35 F \sqrt{\frac{W}{E}}
$$

where $E$ is the energy at which we require the energy resolution (FHWM), $w$ the average energy for electron-hole creation in the specific material and $F$ is a quantity specific to the material called the Fano Factor. (35) The Fano Factor is characteristic to the material and is very difficult to measure. An experimental value of $F$ for CZT is found to be 0.089 \pm 0.005 . (37) The Fano factor has no units. 


\subsubsection{Differences between scintillation and solid state detectors}

The introduction of cameras with solid-state based detectors offers improvements in many imaging aspects compared to the typical cameras with scintillation detectors currently used in SPECT.

The camera used in this study is the Discovery NM 530c manufactured by GE Healthcare and situated at the University of Ottawa Heart Institute. The camera is shown in figure 1.8. Unlike past SPECT cameras, the CZT camera is stationary. The detectors are arranged in a 90-degree arc around the patient. The field-of-view of the camera is a $19 \mathrm{~cm}$-diameter spheroid on the interior of this arc such that a complete set of angles required for reconstruction can be obtained without having to rotate the gantry. Each detector is $4 \mathrm{~cm}$ by $4 \mathrm{~cm}$ with each pixel being $2.46 \mathrm{~mm}$ by $2.46 \mathrm{~mm}$. The system contains 19 detector heads, where each detector head contains 4 detectors in a $2 \times 2$ array, resulting in dimensions of $8 \mathrm{~cm}$ by $8 \mathrm{~cm} .(38)$

The camera acquires list-mode data and collects counts for energies ranging between $40 \mathrm{keV}$ and $255 \mathrm{keV}$. In list-mode acquisition, the incoming information on the position of the photon is digitized, but not immediately stored into an image. Instead, the position information for each individual event along with the time during the acquisition at which it occurred and the energy measured for the incident photon are stored. This permits retrospective framing (sorting of the $\mathrm{x}-\mathrm{y}$ location of individual events into an image grid) of images and more flexibility in choosing energy windows. 


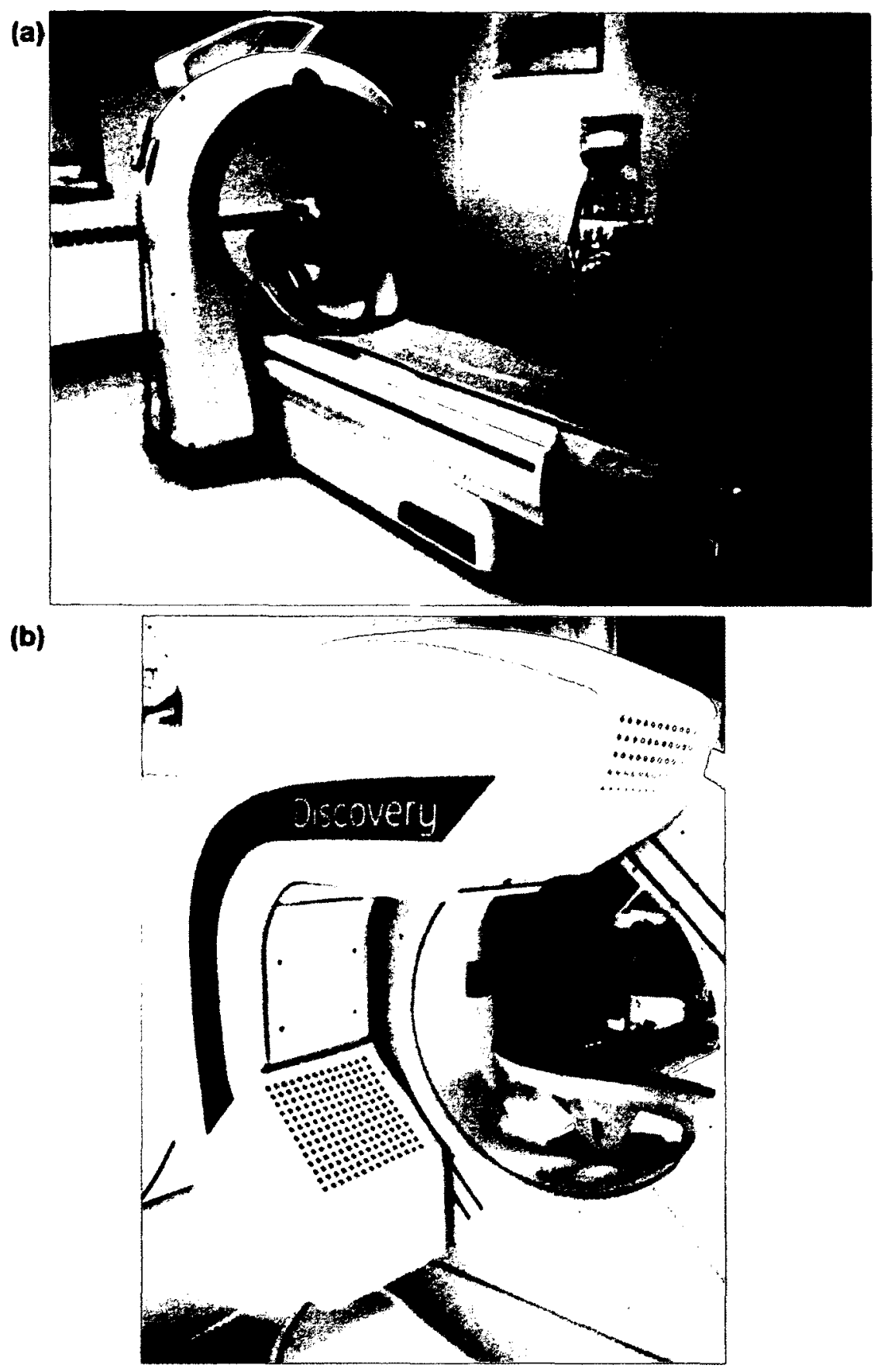

Figure 1.8 Discovery NM 530c from GE Healthcare. (a) Patient bed (b) Stationary detector arc. 


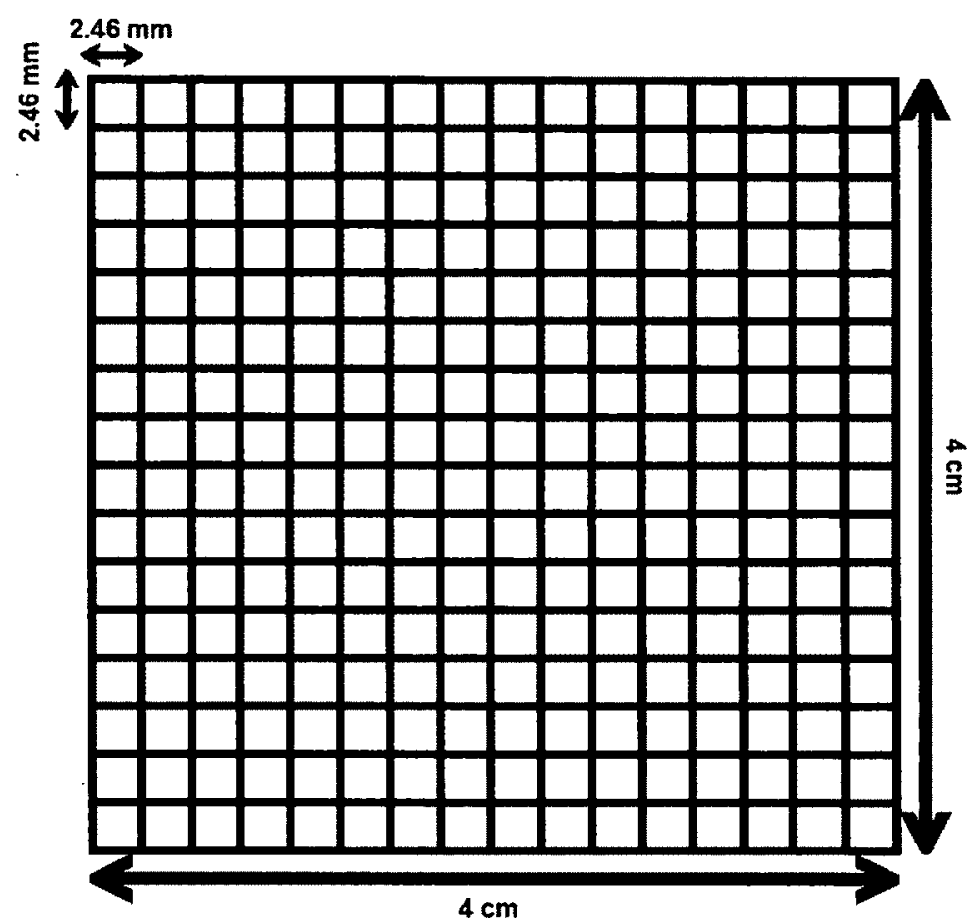

Figure 1.9 CZT detector. Each of the 19 detector heads in the system contains 4 of such detectors in a $2 \times 2$ array, which are $4 \mathrm{~cm}$ by $4 \mathrm{~cm}$. Each detector contains 1024 pixels $(2.46 \mathrm{~mm} \times 2.46 \mathrm{~mm})$

In SPECT, energy discrimination of photons is possible. The primary photons will be detected with a distribution of energies centered on a specific photopeak energy and the width of this peak is determined by the energy resolution of the detector. By choosing to limit the counts included in the image to those within a energy-window range around the primary photon energy, we can create an image which should contain mostly primary counts and eliminate most of the scattered counts acquired by the detector. $(16,34)$ 
After the initial image acquisition, the list file for each patient is stored on the system and can be reprocessed to extract any required energy windows. It is therefore possible to go back and create images for whichever energy window is desired with Lister (GE Healthcare) software included with the CZT camera developed to reframe images containing only counts from energy windows as specified by the user. Within Lister, one is able to choose the central value of the desired primary window with great accuracy. However, the upper and lower boundaries of the window are set as a percent of the central energy. The values of the upper and lower percentages are limited to integer values.

Cadmium-Zinc-Telluride (CZT) detectors offer improved energy resolution over the $\mathrm{NaI}$ detectors currently used. The energy resolution of $\mathrm{NaI}$ detectors for $140 \mathrm{keV}$ photons is $10 \%$. We see an improvement to $6 \%$ in the new CZT detectors. (28) There is also a fourfold improvement of the sensitivity of the CZT detector, compared to NaI detectors currently used. (38)

\subsubsection{Low Energy Tail of the CZT Detector}

The CZT detector is divided into pixels as described previously in section 1.5.3. The charge collection at the boundaries of these elements is not as good as it is in the center of each pixel. Also, the generated signal depends on the depth of the interaction because of electron-hole trapping. (34) Finally, there is an induced signal for interactions close to the collection anode dependent on the relatively slow hole drift due to the finite 
size of the signal contact. All these effects combine to create a signal from primary photons of lower energy than the primary energy window. Figure 1.10 shows spectra of Tc99m and T1201 acquired with the Discovery NM 530c compared to spectra obtained with a NaI detector (Infinia SPECT/CT camera). The counts present in the Tc99m spectrum acquired with Infinia in the $70 \mathrm{keV}$ to $110 \mathrm{keV}$ range are caused by a variety of different sources. The peak seen at approximately $80 \mathrm{keV}$ is most likely due to characteristic lead $\mathrm{x}$-rays occurring in the collimator. This peak is caused by photoelectric interaction of the incoming rays in the lead collimator, leading to emissions of characteristic lead x-rays in the range of $80 \mathrm{keV}$ to $90 \mathrm{keV}$. (16) Another structure appearing in figure 1.10 is the rather large bump centered around $100 \mathrm{keV}$, which is possibly due to backscatter in the detector crystals or even the collimator. The backscatter peak for $\mathrm{Tc} 99 \mathrm{~m}$ occurs at $91 \mathrm{keV}$, strongly suggesting that the peak seen is in fact due to backscatter. (16)

\subsection{Simultaneous dual-isotope imaging}

Simultaneous dual-isotope imaging presents many advantages compared to its single-isotope counterpart. Simultaneous dual-isotope image acquisition would greatly reduce the total imaging time. The total time to complete a single-isotope rest/stress imaging protocol can reach several hours, as illustrated in the American Society of Nuclear Cardiology (ASNC) imaging protocol guidelines (2), due to a long period of waiting between stress and rest image acquisitions to reduce interference between the two images. A typical single-isotope Tc99m rest/stress imaging protocol is shown in figure

\subsection{1 .}



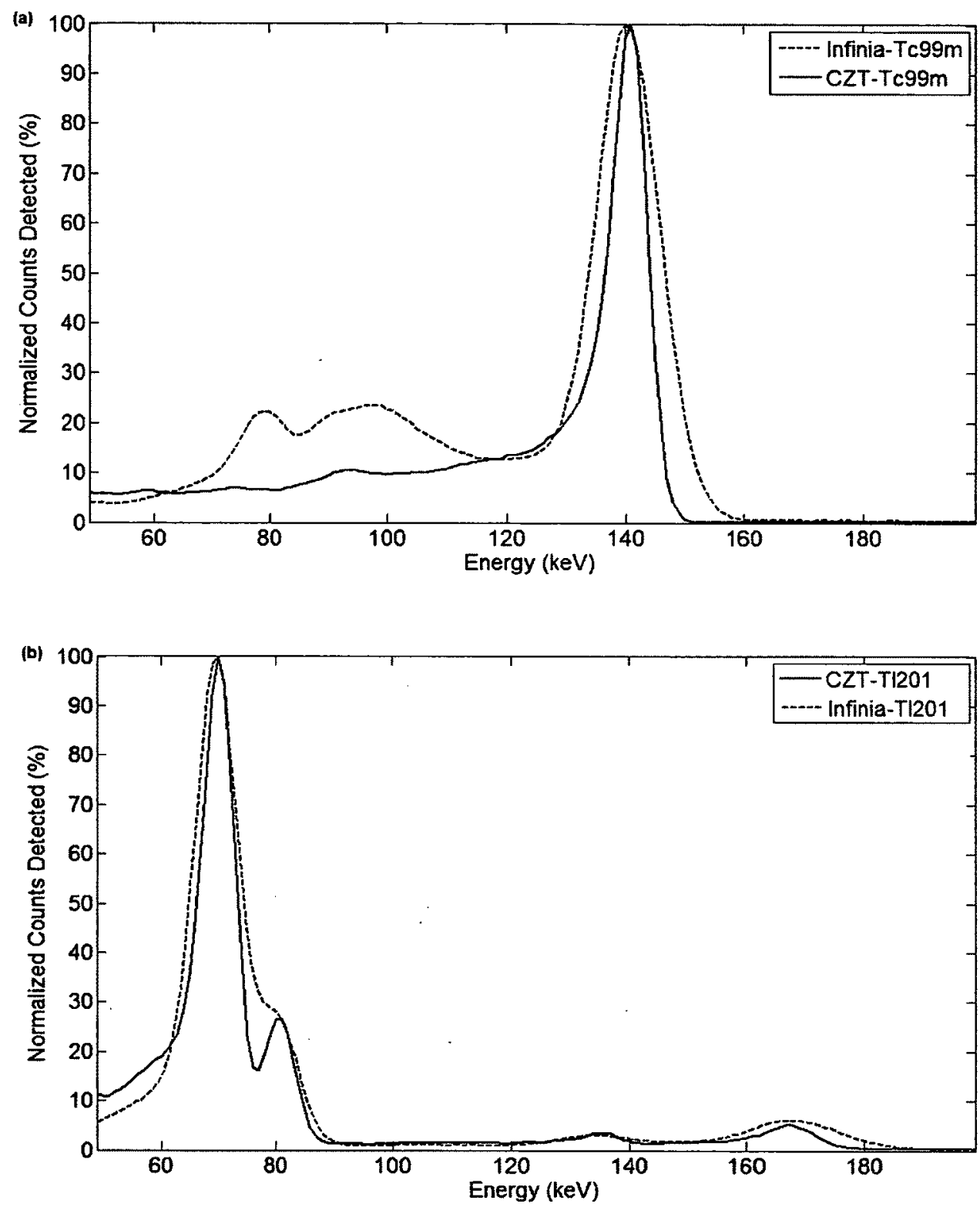

Figure 1.10 Point source spectra for (a) Tc99m and (b) T1201 acquired with Discovery NM 530c and with the Infinia SPECT/CT Scanner for comparison. Spectra were acquired in air with the collimators attached. We clearly see the presence of better energy resolution and the low energy tail of the CZT. 


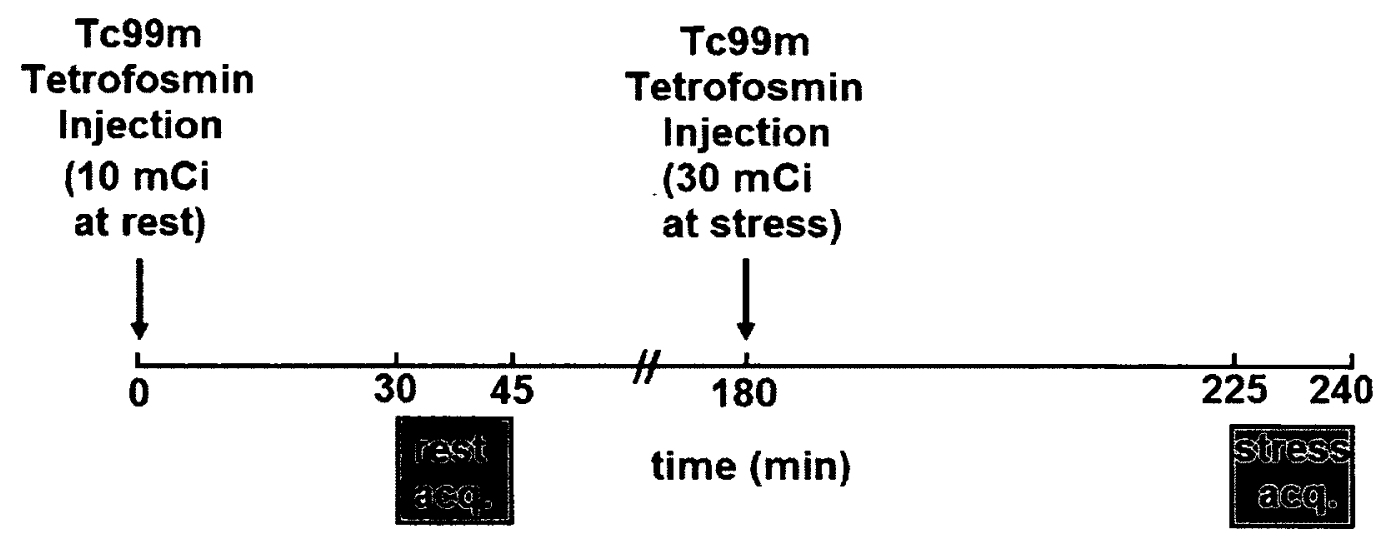

Figure 1.11 Standard 1-day single isotope Tc99m protocol currently being used clinically for rest/stress MPI (time not to scale). Using one isotope for both rest and stress images requires a lengthy wait period between image acquisitions to reduce interference between rest and stress images. This protocol is based onaverage time values taken from the ASNC Imaging Guidelines (2). The stress. state of the heart can be achieved through exercise or induced pharmacologically.

Current protocols being explored reduce the total imaging time from 4-5 hours to less than 30 minutes, improving both patient experience and camera efficiency. Figure 1.12 shows such a protocol. 


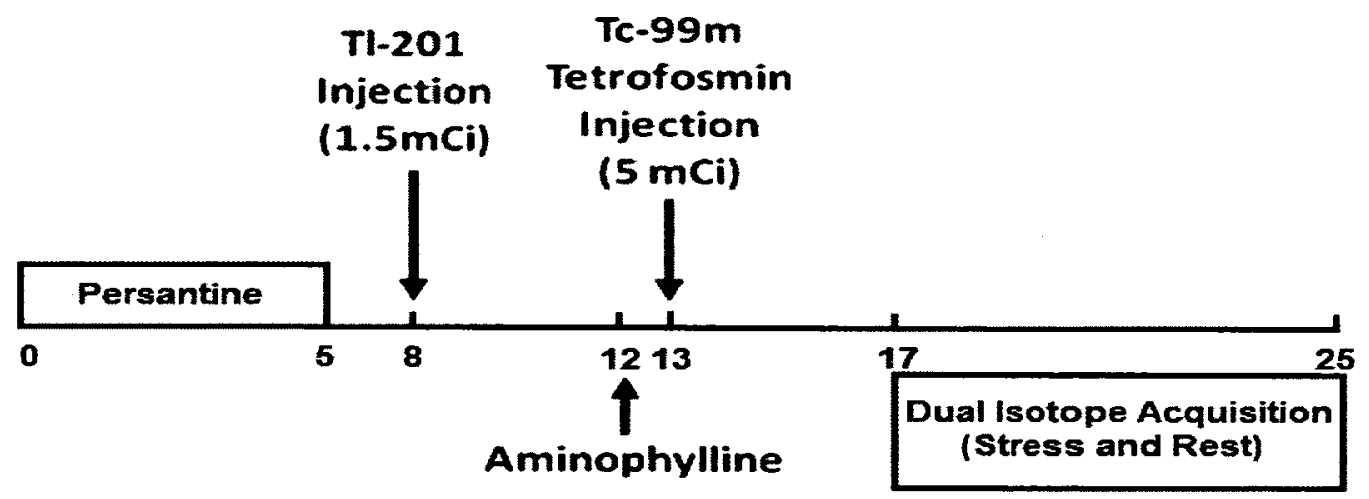

Figure 1.12 Simultaneous acquisition $\mathrm{Tc} 99 \mathrm{~m}$ rest/T1201 stress 30 minute dualisotope protocol for the CZT dedicated cardiac camera. Persantine is a drug injected (dipyridamole) into the patient which induces the heart into a stressed condition. Aminophylline is used to counter the effects of persantine, returning the heart into its rest condition.

Simultaneous dual-isotope imaging only requires one patient setup, which is another advantage over standard single isotope protocols, and produces exact image alignment between the rest and stress images because the patient does not move between the two acquisitions.

\subsubsection{Complications in simultaneous dual-isotope imaging}

Even with all the advantages offered by simultaneous dual-isotope imaging, there are also many complications and problems with the proposed method that have to be tackled in order to see clinical implementation of the associated protocols. 


\subsubsection{High effective dose to patient}

Current dual-isotope protocols provide a very high effective radiation dose to the patient. Current Tc99m stress/T1201 rest dual-isotope protocols deliver an effective dose of $29.3 \mathrm{mSv}$ to the patient. (21) This dose is very high for a diagnostic procedure. For single-isotope protocols with $\mathrm{NaI}$ cameras, the patient receives significantly less effective dose with similar diagnostic accuracy. For a Tc99m-tetrofosmin rest/stress procedure, the patient receives a $9.9 \mathrm{mSv}$ effective dose while a $22.0 \mathrm{mSv}$ effective dose is delivered during a T1201 stress-redistribution protocol. (21)

The improved sensitivity of the CZT-based camera will allow reduced-dose procedures to be performed on patients. The new camera gives us a four-fold improvement of sensitivity over typical SPECT systems and so we can acquire a comparable image in a quarter of the time. The increased sensitivity also means that we can inject less activity for imaging, therefore delivering less effective dose to the patient and lowering the risks associated with the procedure. Current dual-isotope protocols being explored with the CZT camera at the University of Ottawa Heart Institute inject 5.5 $\mathrm{MBq}$ of $\mathrm{T} 1201$ and $185 \mathrm{MBq}$ of Tc99m-tetrofosmin and have reduced the effective dose to the patient to approximately $10 \mathrm{mSv}$, making it comparable to the dose received from Tc99m single isotope procedures. 


\subsubsection{Presence of cross-talk between isotopes}

Another major problem with simultaneous dual-isotope imaging is the presence of cross-talk between tracers due to Compton scatter.

Cross-talk is the presence of Compton-scattered photons from one isotope in the primary energy-window of a second isotope. In this study, the two isotopes proposed for dual-isotope imaging are Tc99m and T1201. We can see the patient spectrum of both of these isotopes in figures 1.13 and 1.14 .

From figure 1.13, it is clear that there is a significant presence of Tc99m counts in the T1201 primary window. These extra counts interfere with the signal from the T1201 tracer. Figure 1.14 shows little contribution from $T 1201$ scatter into the $T c 99 \mathrm{~m}$ primary window. However, corrections can still be done in both windows in order to improve image quality.

\subsection{Scatter corrections}

Many of the techniques that are used to correct for cross-talk in dual-isotope imaging were originally developed to correct for the detection of scattered photons in the photopeak of single-isotope images. Scattered photons account for $30-40 \%$ of the photons detected in the primary energy window of a SPECT system. (39) The detection of scattered photons will result in a loss of contrast in the image and can degrade the image quality by reducing the severity of defects (regions of reduced tracer uptake) and thus decreasing diagnostic accuracy. 


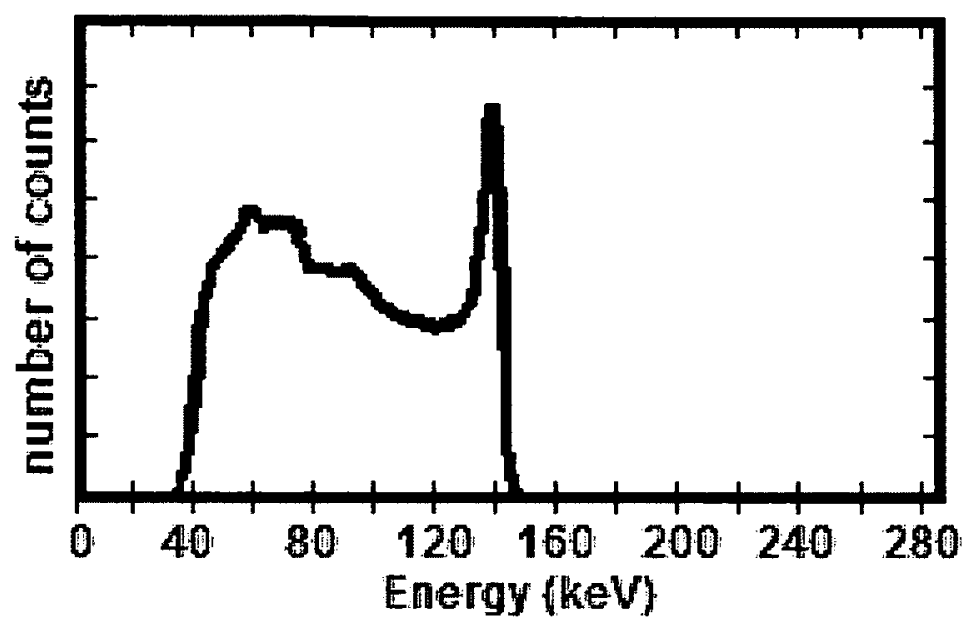

Figure $1.13 \mathrm{Tc} 99 \mathrm{~m}$ patient energy spectrum. Spectrum acquired with Discovery NM 530c (GE Healthcare). The primary energy peak can clearly be seen at 140 $\mathrm{keV}$, but we also notice a large number of scatter counts at lower energies. The scale for the number of counts is linear.

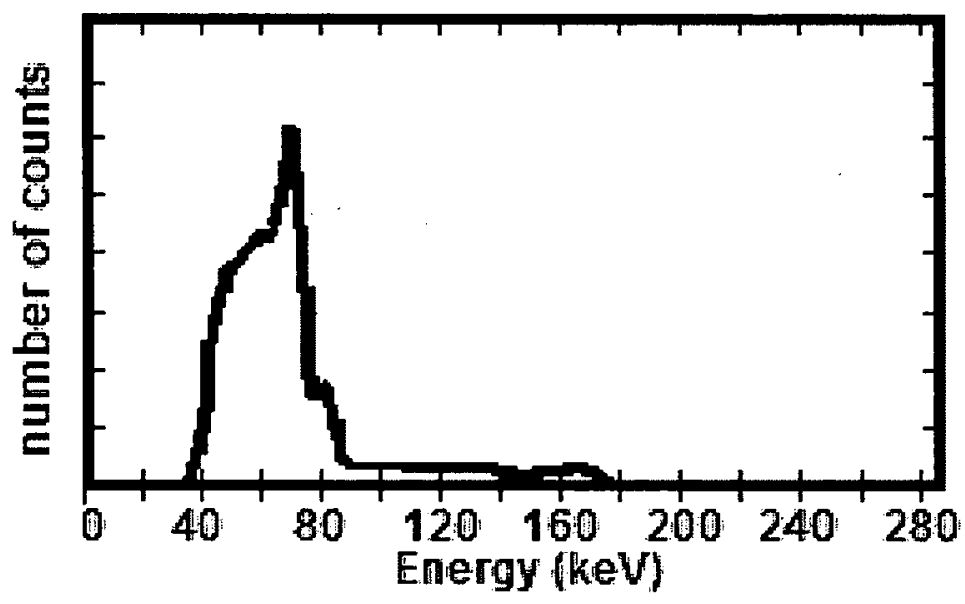

Figure 1.14 T1201 patient energy spectrum aquired with Discovery NM 530c (GE Healthcare). The primary x-ray emissions $(70-80 \mathrm{keV})$ can clearly be seen, as well as a small peak at $167 \mathrm{keV}$. The number of counts are scaled linearly. A large number of scatter counts are also noted in the $40-60 \mathrm{keV}$ range. 
While using energy discrimination can eliminate a lot of the counts from scattered photons, there is still a significant number of counts in the primary energy window from scattered photons, even when only using one isotope. Scatter is more of an issue with the application of attenuation correction in images. (40) The effects of attenuation are greater than the effects of scatter but removal of attenuation artifacts can highlight artifacts caused by scatter. Thus scatter corrections are usually applied in conjunction with corrections for attenuation. (41)

There have been many methods developed to remove the scattered counts from single-isotope images which will be discussed in further sections. When working with multiple isotopes, the presence of Compton scatter becomes more of an issue, due to the presence of cross-talk. Cross-talk refers to the detection of photons originating from one isotope in the energy-window of the second isotope.

Several methods have previously been developed for correction of self-scatter in single-isotope image acquisitions. $(39,42,43)$ Some of these corrections have been based on direct measurement of the scatter (44-49). while others concentrate on developing ways to accurately model the scatter distribution in the images (50-58) or use methods such as convolution subtraction. $(59,60)$

\subsubsection{Scatter Correction Based on Modeling the Scatter Distribution}

Various methods have been developed to correct for scatter by calculating the scatter distribution through modeling the probability of photon scattering based on the 
underlying principles of scattering interactions. (50-58) Scatter is modeled using various methods, often Monte-Carlo based, and subtracted before reconstructing the images. (39) The modeling is done by calculating the probability of interaction at each point of each possible photon path through the patient to determine which photons (of specific energy) scatter, reach the detector and are registered. The probability of interaction is determined by the Klein-Nashina equation (describing the differential cross-section of Compton scatter interactions). Some methods included the scatter estimation in the reconstruction itself. (53,56-58) In these methods, scatter compensation is achieved by mapping scattered photons back to their point of origin.

Computation time for Monte Carlo based methods is excessive and the scatter calculations need to be done specifically for each patient, making it very time consuming and unappealing for daily clinical use. However, accelerated Monte Carlo methods have been developed using approximations, making computation times quite quick. (61) These are based on multiple approximations and therefore produce a scatter estimation that may be less accurate than those achieved by non-accelerated Monte Carlo methods.

Methods based on analytical integration of the Klein-Nishina equation have also been developed, but the equation has no analytical solutions, resulting in the need for approximations and a loss of accuracy in the scatter estimations. (50)

Other methods estimate scatter using patient-dependent scatter kernels. One method first determines the scatter response kernel for a point source behind slabs of different thicknesses of water and later samples this information to model variously 
shaped objects. $(50,55,56,62)$ The point source is moved to different locations behind the slabs and a table of coefficients is produced that can be later consulted knowing patient geometry to create patient specific scatter estimates. This method is sometimes referred to as slab derived scatter estimation (62). The Effective Scatter Source Estimation (ESSE) (55) method uses Monte Carlo derived scatter kernels to propagate an activity distribution to the point of last scatter in the patient, creating an effective scatter source. This scatter source is added to the activity distribution in the reconstruction process to estimate the full projection data.

For all of the modeling methods, the source of all activity in the image must be known. However, with the CZT system, the field of view (FOV) is truncated to the heart region. Many isotopes used in SPECT tend to settle in other organs that are not located in this FOV, but can still contribute to the measured scatter such as liver, intestines and sometimes lungs. Since these organs are not included in the image, these scatter counts will therefore not be accounted for in the modeling estimations, since we do not know the geometry of the structures responsible for the scattered photons appearing in the images.

\subsubsection{Convolution Based Scatter Corrections}

Another subset of scatter correction methods is the convolution based methods. In these methods, the scatter $(S(x))$ is estimated by convolving the image $(P)$ with a certain scatter kernel $(F)$ over the width of the image (2D).

$$
S(x)=\int_{-D}^{D} P(\tau) * F(x-\tau) d \tau
$$


The scatter distribution will strongly depend on the properties of the detector being used and thus the scatter kernel should be chosen accordingly. The scatter estimate $\mathrm{S}$, which is essentially a blurred version of the original image, is then subtracted from the original image in order to complete the scatter correction. $(60,63)$

A difficulty with equation 1-7 is that the scatter kernel is not spatially invariant (depends on the position) and is depth dependent. Therefore, a multitude of scatter kernels are needed to properly create the scatter estimate from this method. In addition, we again run into the problem of out of FOV scatter, as with the modeling approaches, because the images from the CZT with its truncated FOV contain scattered photons from sources that are outside the FOV.

\subsubsection{Energy Based Scatter Corrections}

There are many methods developed for scatter compensation that rely on direct measurement of scatter from the acquired data. (44-51) Most of these methods estimate scatter based on parts of the energy spectrum where most of the counts are from scattered photons. One such method performs factor analysis of dynamic structures (FADS) on the entire energy spectrum. Factors are extracted using matrix algebra on a data set of a certain number of energy-window images arranged in matrix form which can then be associated with the spectrum of primary photons and the spectrum of scattered photons. Each row of the matrix represents the variation of the number of counts recorded in a pixel throughout all the energy images. (51) 
This method requires a dense sampling of energy windows in order to provide accurate scatter estimates. The greater the number of scatter windows used in the corrections, the more accurate it will be. The typical cameras used clinically do not have access to list-mode data and are limited to very few energy windows (typically 4-5) per image acquisition. One of these windows must be reserved for the primary image, leaving but a few scatter window options. For cameras which acquire list-mode data, the process for these corrections would be lengthy, with the need to extract a great number of energy windows. The math behind the correction, while yielding accurate scatter estimates when done correctly with an adequate amount of scatter windows, is very complicated and much simpler scatter correction methods have been explored.

\subsubsection{Triple Energy Window scatter correction method}

We have focused our research on an energy-based scatter correction called the triple energy window (TEW) method. This involves a simple algorithm providing a scatter estimate based on the counts in discrete energy windows situated directly above and below the primary energy window. These windows will be referred to as our scatter windows. Having access to list-mode data means that we have complete freedom to select the energy windows that will be used. There can be issues with noise propagation with the TEW method. In order to create accurate estimates of the spatial distribution of scatter in the primary window, the energy windows used to create the estimates must be quite narrow, meaning working with very low count statistics leading to a noisy scatter estimate. Also, a subtraction is required in order to complete the correction with TEW, 
introducing noise into our primary image. Nevertheless, this method has been shown to be effective for single isotope studies using $\mathrm{NaI}$-based cameras. $(44,46)$

The amount of scattered photons in the primary window can be estimated as

$$
C_{s}=\left(\frac{C_{a}}{w_{a}}+\frac{C_{b}}{w_{b}}\right) * \frac{w_{p}}{2}
$$

where $C_{s}$ is the counts estimated to be scatter, $C_{a}$ and $C_{b}$ are the counts in the lower and upper scatter windows of width $w_{a}$ and $w_{b}$ and $w_{p}$ is the width of the primary window. A graphical representation of the energy windows used in TEW is shown in figure 1.15.

The number of counts from scattered photons in each pixel can be estimated from equation 1-8. In order to correct for scatter, a simple subtraction of the estimate from the original image $\left(I_{o}\right)$ is required on a pixel by pixel basis, resulting in an image containing mostly primary photons $\left(I_{p}\right)$.

$$
I_{p}=I_{o}-C_{s}
$$

\subsubsection{Cross-talk Correction Methods in Dual-Isotope Imaging}

Dual-isotope MPI would present a great improvement in image acquisition time, improving camera efficiency and overall patient experience. As previously discussed, an obstacle to multi-isotope imaging is the presence of cross-talk. Many scatter correction methods have been applied to dual-isotope studies with traditional cameras $(5,64)$ including the Tc99m-T1201 isotope combination that we focus on in this work $(10,12,65)$. 


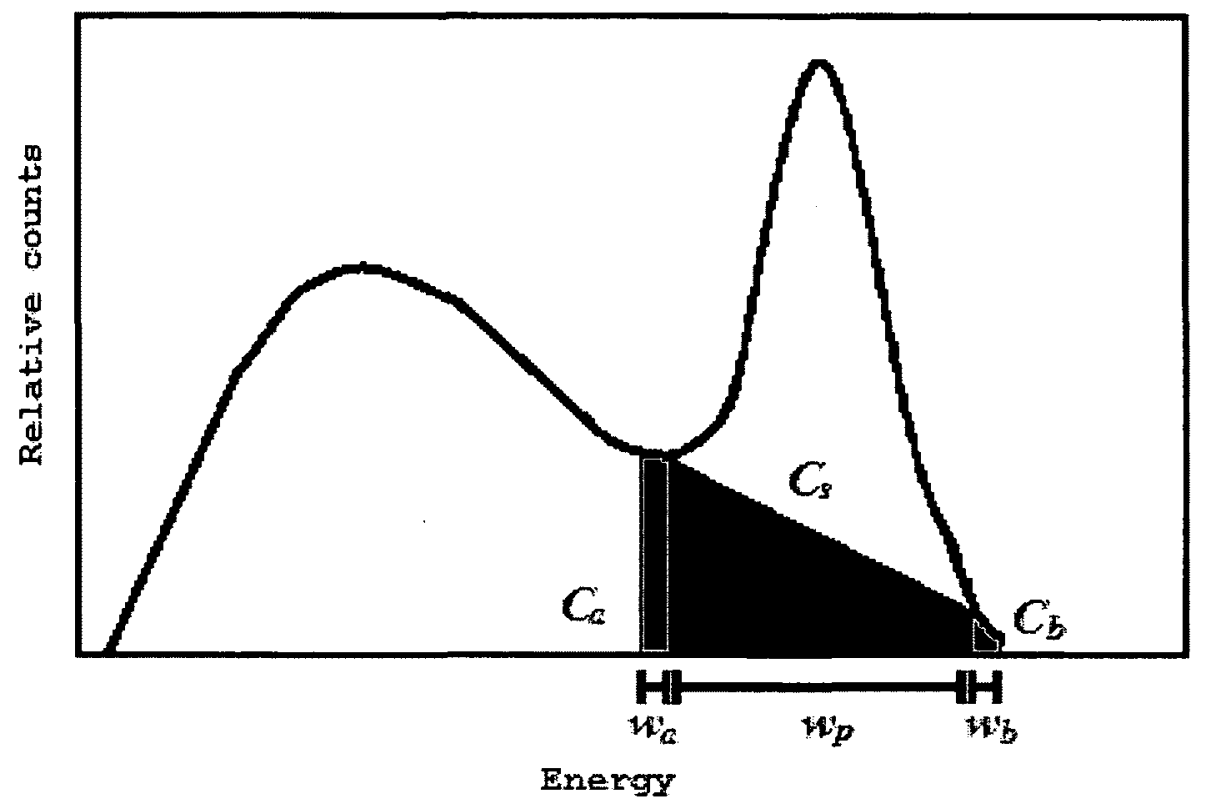

Figure 1.15 Graphical representation of windows used in TEW. $C_{a}$ and $C_{b}$ are the counts in lower and upper scatter windows respectively (of width $w_{a}$ and $w_{b}$ ) while $C_{s}$ represents the number of counts estimated to be scatter in the primary window of width $w_{p}$.

There are, however, very few studies addressing dual-isotope imaging with CZT-based dedicated cardiac cameras. These new cameras have improved energy resolution and sensitivity over traditional cameras used clinically. Better energy resolution leads to the ability to better discriminate between primary and scattered photons while improved sensitivity leads to less noise.

In a recent study by Ben-Haim et al. (3), dual-isotope imaging was studied on a different type of dedicated cardiac camera that uses the same CZT-based solid-state 
detectors (D-SPECT, Spectrum Dynamics). Cross-talk correction was studied using a spillover correction. For each primary peak studied, a scatter peak situated directly below the primary peak was also selected. Each of these windows selected is assumed to contain counts due to primary photons, photons from cross-talk as well as counts detected with reduced energy due to characteristics of the detector. The method was based on one previously developed (11). First, the method uses point sources to establish models for downscatter and spillover effects (low energy tail) specific to the system. The scatter models used a point source in water while the spillover effects used a point source in air. Second, the images are acquired in different energy windows (primary and scatter window). Third, a set of equations is solved (one for each window present) using iterative maximum likelihood to estimate primary counts in the photopeak window and finally to reconstruct the projections which should be free of cross-talk counts. Although this study demonstrated some success, we wanted to determine if the simpler TEW approach could also provide an adequate correction. Figure 1.16 shows the windows to be used for TEW corrections in simultaneous dual-isotope imaging.

\subsection{Objectives of the thesis}

The TEW method is very appealing for clinical implementation as the simple subtraction-based correction would only take seconds to correct each image. However, the method has yet to be validated in simultaneous dual-isotope imaging with the dedicated cardiac CZT camera used in this study. 


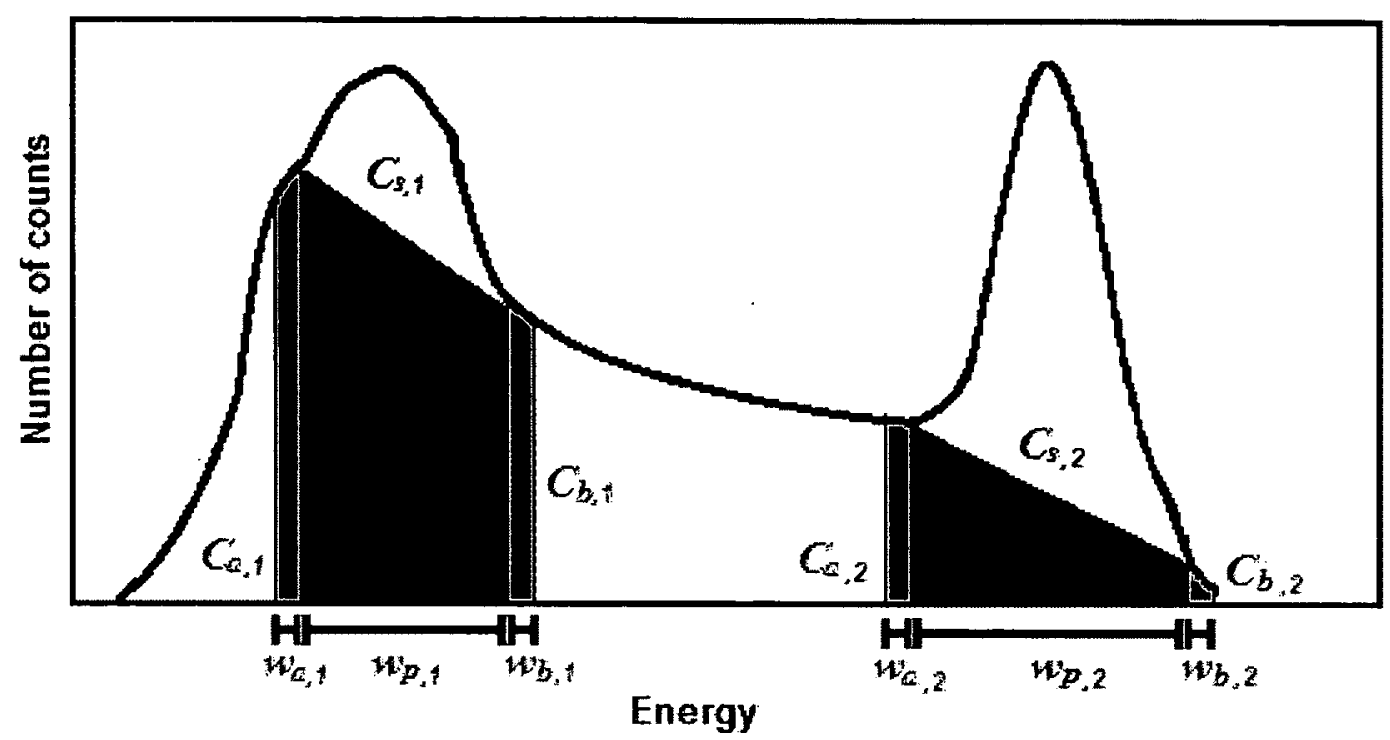

Figure 1.16 Graphical representation of windows used in TEW for dual-isotope imaging. $C_{a, i}$ and $C_{b, i}$ are the counts in lower and upper scatter windows respectively with corresponding widths $w_{a, i}$ and $w_{b, i} . C_{s, i}$ represents the number of counts estimated to be scatter in the primary window of width $w_{p, i}$. The index $\mathrm{i}$ indicates the isotope (either isotope 1 or 2 ) for which scatter is being estimated.

The objective of this study was to determine the quantitative and qualitative accuracy of a modified energy based cross-talk correction in dual-isotope SPECT imaging with a dedicated cardiac CZT-based camera. Modifications to the method were needed in order to avoid different characteristics of the spectrum presented by the CZT detectors. These modifications will be explained in the next chapter. All corrections are done in image space (post-reconstruction), because we do not have access to offline image reconstruction. The corrections are done after all images are exported from the GE 
discovery software and therefor do not have to be reimported for reconstruction before analysis.

The author's principal role was to perform the modified TEW correction on the patient data sets provided followed by quantitative and qualitative analysis to determine the accuracy of the correction. All images required to perform the correction were acquired from the list-mode data that were archived at the University of Ottawa Heart Institute (UOHI). The list-mode data for each patient were acquired between 2009 and 2010 by imaging technicians employed at the UOHI. The author created primary images to correct as well as scatter images to create scatter estimates from the list-mode data. The scatter was estimated and corrections applied by the author. The author wrote all code for the corrections applied using MATLAB. The code involved importing files in the program, creating scatter estimates from different scatter windows acquired and corrections of the primary image. Volume of interest (VOI) containing only heart data for quantitative analysis were selected on a patient by patient basis by the author. The resulting images were analysed by the author as described in the next chapter. Mean bias and pixel standard deviation were calculated using both code written by the author and packaged statistical functions in MATLAB. Results for bias and pixel standard deviation were imported into Microsoft Excel for the author to create graphical representation of the results and for performance of statistical tests to determine significance of the results of this study. 


\section{Chapter 2: Cross-talk Correction with a Dedicated Cardiac Camera}

\subsection{Introduction}

\subsubsection{Motivation for dual-isotope imaging}

Heart disease remains one of the main causes of death in Canada, responsible for $29 \%$ of all mortalities in 2008. (1) SPECT offers a wide range of radioactive isotopes for MPI. As long as we have equipment allowing us to do so and good energy discrimination, the use of multiple isotopes acquired simultaneously is possible with this imaging modality. Serial dual-isotope protocols have been studied for T1201/Tc99m MPI.

$(4,5)$ The total time for this suggested protocol is reduced to about $60-90$ minutes. (2) Simultaneous dual-isotope protocols would further reduce the total time for the procedure 
to approximately 30 minutes, $(3,10)$ therefore improving patient experience and camera efficiency even more than with serial dual-isotope imaging. Image alignment of the rest and stress images is also improved by doing simultaneous imaging.

\subsubsection{Complications in multi-isotope imaging}

T1201 and Tc99m are two isotopes presently used in myocardial perfusion imaging. With primary emissions around $70 \mathrm{keV}$ and at $140 \mathrm{keV}$ respectively, $(20,22)$ these two isotopes can be discriminated with adequate energy resolution and are the two isotopes typically chosen for dual-isotope cardiac imaging.

Dual-isotope imaging brings up complications that must be surpassed before it can be clinically implemented. The total effective dose to the patient for the ASNC approved serial $\mathrm{Tc} 99 \mathrm{~m}$ rest/T1201 stress imaging protocols is on the order of $29 \mathrm{mSv}$ (21), which is very high for a diagnostic test. Equally important is the presence of crosstalk.

Scattered photons from one of the isotopes can cause a significant amount of interference in the opposing isotope's primary energy window, causing a severe degradation in image quality and diagnostic accuracy. This work concentrates on correction for this interference between tracers. 


\subsubsection{CZT camera}

Dual-isotope imaging and the correction for cross-talk has been explored previously. $(3,10,39)$ Recently, dedicated cardiac cameras have been introduced for clinical use. These new designs offer a number of potential advantages for performing dual-isotope SPECT. One of these new systems is the Discovery NM 530c camera (GE Healthcare) based on Cadmium-Zinc-Telluride (CZT) solid-state detectors. The Discovery NM 530c offers improved sensitivity and energy resolution compared to the Sodium-Iodine (NaI) scintillators typically used in diagnostic SPECT imaging. $(26,27)$ The detectors are solid-state based, which offer advantages compared to the typical NaI scintillator based cameras currently used in the clinic.

Reduced-dose protocols are being developed for use with the CZT. The CZT detector offers a 4-fold improvement on sensitivity compared to the $\mathrm{NaI}$ detectors (28) Image acquisition and injected activity can be reduced while keeping the same quality image as with previous protocols. The dual-isotope protocols being developed at the University of Ottawa Heart Institute for image acquisition with the new camera use injections of $55 \mathrm{MBq}$ for $\mathrm{T} 1201$ and $185 \mathrm{MBq}$ for Tc99m-tetrofosmin, delivering a total effective dose to the patient of approximately $10 \mathrm{mSv}$, which is much more reasonable than the $29 \mathrm{mSv}$ from the dual-isotope protocol described previously.

The camera also offers an improvement in energy resolution. The resolution of the CZT detector is $6 \%$ at $140 \mathrm{keV}$. At this energy, the NaI detectors offer an energy resolution of $10 \% .(26)$ 


\subsubsection{Objective}

The objective of this study is to determine the quantitative and qualitative accuracy of a TEW cross-talk correction in dual-isotope SPECT imaging with CZT.

\subsection{Methods}

\subsubsection{Tracers}

The two tracers used in this study are Technitium-99m (Tc99m) tetrofosmin and T1201 - thallium-chloride. Tc99m is widely used in myocardial perfusion imaging, with easily detectable gamma emissions at $140 \mathrm{keV}(88.5 \%$ abundance) and a half-life of 6.02 h (20). This combination of half-life and primary energy allows sufficient time for image acquisition while keeping dose to the patient low. Typical effective dose to the patient from a single-isotope tetrofosmin rest/stress one-day protocol is $9.9 \mathrm{mSv} .(21)$

Thallium-201 is the other isotope used in this study. Its main energy peak is a collection of $\mathrm{Hg}$ x-rays, ranging in energy between 70 and $80 \mathrm{keV}$, with emission abundance of $94.4 \%$ (22). It also has emissions of $135 \mathrm{keV}$ and $167 \mathrm{keV}$ gamma rays with $2 \%$ and $8 \%$ abundance, respectively. The typical effective dose to the patient from a T1201 stress-redistribution study is $22 \mathrm{mSv}(21)$.

\subsubsection{Patient set}

This study is done using single isotope images acquired at standard injected activities with the CZT camera. All patients signed informed consent to allow use of their 
data as approved by the University of Ottawa Heart Institute Research Ethics Board. The patient set includes a total of 104 patients, 52 of which were imaged with a rest/stress Tc99m full-dose clinical protocol, while the other 52 were imaged with a full-dose clinical stress/redistribution T1201 protocol. The patients received Tc99m injections of $350 \mathrm{MBq}$ at rest and $1100 \mathrm{MBq}$ at stress or a T1201 injection of $130 \mathrm{MBq}$. Image acquisitions times were 3 minutes for stress images and 5 minutes for rest or redistribution images. These activity amounts and image acquisition times are in accordance with the recommended guidelines of the manufacturer and confirmed as appropriate by others (30). The patient sample represents a wide range of body-mass indexes (BMI) but is evenly divided in gender, with a total of 26 males and 26 females for each tracer involved. Table 2.1 shows the patient demographics.

Table 2.1 Patient demographics. The patient set includes 52 males and 52 females.

\begin{tabular}{|l|c|c|c|c|}
\hline & $\begin{array}{c}\text { Age } \\
(\text { years) }\end{array}$ & $\begin{array}{c}\text { Height } \\
(\mathbf{c m})\end{array}$ & $\begin{array}{c}\text { Weight } \\
(\mathbf{k g})\end{array}$ & $\begin{array}{c}\text { BMI } \\
\left(\mathbf{k g} / \mathbf{m}^{2}\right)\end{array}$ \\
\hline Mean & 63 & 168 & 79 & 27.9 \\
\hline Std. Dev. & 10 & 9 & 15 & 4.5 \\
\hline Max. & 89 & 190 & 111 & 37.2 \\
\hline Min. & 42 & 149 & 45 & 16.5 \\
\hline
\end{tabular}

A set of simulated dual-isotope patients was created from single isotope acquisitions. A total of 26 simulated patient studies were created as part of a previous 
study on cross-talk (67) and the same studies were used in this work. To create each image set, a pair of patients were identified from our database of archived studies at the University of Ottawa Heart Institute. Patients were matched by gender and BMI with the expectation that this might generate more accurate estimates of cross-talk contamination. Each simulated patient is composed of the $\mathrm{Tc} 99 \mathrm{~m}$ rest acquisition from one patient and the T1201 stress acquisition from the other patient. The total set of dual-isotope patients is composed of 26 males and 26 females. The images were created by summing the counts from the two sets of projections on a pixel-by-pixel basis. By simulating dual-isotope studies in this fashion, we were able to compare the corrected images to the original single isotope acquisitions, which were not contaminated and were therefore taken as "truth".

\subsubsection{Cross-talk compensation - Triple Energy Window method}

Cross-talk is one of the major roadblocks in the clinical implementation of dualisotope imaging. Scatter correction in single-isotope SPECT imaging has been widely explored in the past few decades. Many methods have been developed, proving the possibility to greatly reduce self-scatter, and these methods are also useful for the reduction of cross-talk $(10,46,51)$. However, many of these methods are based on scatter modeling that can be very complex and time-consuming. (50-58) With the goal of clinical implementation, the focus is to find means to eliminate cross-talk that is very quick and simple. Developed by Ogawa et al. in 1991, the Triple Energy Window method (TEW) meets these criteria. The correction is based on using information from the energy 
spectrum and knowledge of the behavior of photons due to cross-talk to estimate the amount of cross-talk in the principal window. $(44,46)$

TEW correction uses two small scatter windows on either side of the photopeak. Smaller windows more accurately estimate the spatial distribution of the scattered photons but are subject to increased noise from poor count statistics. To evaluate this trade-off for the CZT camera, we investigated 4 different scatter window widths for each correction applied. Also explored in this study was the position of the scatter windows relative to the primary energy window. All corrections were done in image space (postreconstruction) to facilitate processing.

The study was divided into three phases. The first two phases of the study dealt with assessing the accuracy of cross-talk estimates in the primary energy windows. In phase one, an image of the primary Tc99m window containing only Tl201 counts was created from the T1201 single-isotope study in order to evaluate the accuracy of cross-talk estimation in this window. Phase two involves evaluation of the method in the Tl201 window using only $\mathrm{Tc} 99 \mathrm{~m}$ counts. These two first phases allow determination of several modifications needed in order to optimize the correction of these images. The third phase explored the TEW method with the modifications from the previous phases on the simulated dual-isotope studies. 


\subsubsection{Image Creation}

In order to correct the primary images for cross-talk, a number of different images are needed. The camera used in this study acquires list-mode data, from which different projection datasets can be formed containing photons from any energy windows within the limit of acquisition energies.

The software allowing us to create our images from the Discovery NM 530c is called Lister. Lister is a very useful tool allowing us to take the list-mode data acquired by the camera and form images. Images are formed from the projection data in a certain energy window. The center energy value (in $\mathrm{keV}$ ) and boundaries of the energy window (in \% of the center value) can be selected to form projection data containing only counts in the specific energy windows. As previously described in sections 1.7 .3 .1 and 2.2.3, for TEW corrections two scatter windows above and below the primary window are used. The values for all energy windows used in this study are given in tables $2.3-2.5$.

Before the correction is applied, all images are reconstructed within Lister with the use of the included reconstructed tool. The reconstruction is done with MLEM as described in section 1.4.3.

\subsubsection{Scatter fractions}

Scatter fractions were calculated in order to evaluate the presence of cross-talk in both the Tc99m and T1201 windows. These scatter fractions are shown in table 2.2 for both male and female patients. 
Table 2.2 Scatter fractions in Tc99m rest/ Tl201 stress study

\begin{tabular}{|l|c|c|}
\hline & $\begin{array}{c}\text { Tc99m cross-talk in } \\
\text { T1201 window (\%) }\end{array}$ & $\begin{array}{c}\text { T1201 cross-talk in } \\
\text { Tc99m window (\%) }\end{array}$ \\
\hline Males & $30.4 \pm 7.7$ & $6.4 \pm 2.3$ \\
\hline Females & $25.2 \pm 8.3$ & $10.9 \pm 11.2$ \\
\hline
\end{tabular}

\subsubsection{Phase 1 - Estimation of T1201 cross-talk in the Tc99m primary window.}

The contamination of $\mathrm{T} 1201$ in the $\mathrm{Tc} 99 \mathrm{~m}$ is only on the order of $10 \%$ (see table 2.2), but nevertheless present. The need for an accurate correction is not as great as it is in the T1201 window and shall be explored in this section. The T1201 cross-talk was estimated using the TEW method.

\subsubsection{Energy windows considered}

Clinically, the Tc99m images are obtained using a symmetrical window centered at $140 \mathrm{keV}$ with a width of $10 \%$. However, for the purposes of this study, a symmetrical window of $6 \%$ was chosen about the central energy of $140 \mathrm{keV}$, to reflect the improved energy resolution of the CZT detector. The motivation behind choosing such boundaries for the primary window is to allow sufficient room for scatter windows above the primary peak, without including counts from the $167 \mathrm{keV}$ Tl201 primary peak in the scatter estimates. 
Several different scatter window widths were considered to find those which gave an accurate correction while minimizing the addition of noise into our images. In Lister, the values of the upper and lower percentages to choose the desired energy windows are limited to integer values, therefore limiting the possible widths of the narrow scatter windows, especially around $140 \mathrm{keV}$. Scatter window widths of approximately $3,5,8$ and $10 \mathrm{keV}$ were considered.

For corrections with the smaller scatter window widths, both the lower and upper scatter windows are approximately the same width. However, for the upper scatter window, the upper limit of this window is limited to $157 \mathrm{keV}$, due to the presence of the T1201 primary peak at $167 \mathrm{keV}$. Therefore, the width of the upper scatter window in both the 8 and $10 \mathrm{keV}$ corrections will be $5 \mathrm{keV}$, using the same upper scatter image as the 5 $\mathrm{keV}$ correction.

The exact values of all window widths used for the TEW estimate of cross-talk into the $\mathrm{Tc} 99 \mathrm{~m}$ primary window are listed in table 2.3 .

\subsubsection{Modifications to TEW}

Modifications to the TEW estimate are needed in order to compensate accurately for cross-talk. A complication arising in this energy window is the presence of primary $\mathrm{T} 1201$ counts from a secondary peak at $135 \mathrm{keV}$, which lies directly in the primary energy window of Tc99m. This results in T1201 primary counts that are not accounted for in the TEW estimate. 
Table 2.3 Energy window information for TEW correction in Tc $99 \mathrm{~m}$ primary window. A $5 \mathrm{keV}$ upper scatter window is used with the $8 \mathrm{keV}$ and $10 \mathrm{keV}$ lower scatter windows to not have the scatter windows overlap with the $167 \mathrm{keV}$ primary T1201 window.

\begin{tabular}{|c|c|c|c|c|c|}
\hline \multicolumn{2}{|c|}{ Energy Window } & $\begin{array}{c}\text { Center } \\
(\mathrm{keV})\end{array}$ & $\begin{array}{c}\text { Width } \\
(\%)\end{array}$ & $\begin{array}{l}\text { Width } \\
\text { (keV) }\end{array}$ & $\begin{array}{l}\text { Range } \\
(\mathrm{keV})\end{array}$ \\
\hline \multicolumn{2}{|c|}{ Primary } & 140 & \pm 6 & 16.8 & $131.6-148.4$ \\
\hline \multirow{2}{*}{$\begin{array}{l}\text { Scatter } \\
(3 \mathrm{keV})\end{array}$} & Lower & 130 & \pm 1 & 2.6 & $128.7-131.3$ \\
\hline & Upper & 149 & \pm 1 & 3.0 & $148.5-151.5$ \\
\hline \multirow{2}{*}{$\begin{array}{l}\text { Scatter } \\
(5 \mathrm{keV})\end{array}$} & Lower & 129 & \pm 2 & 5.1 & $126.4-131.5$ \\
\hline & Üpper & 150 & \pm 2 & 6.1 & $147.9-154.0$ \\
\hline \multirow{2}{*}{$\begin{array}{c}\text { Scatter } \\
(8 / 5 \mathrm{keV})\end{array}$} & Lower & 127 & \pm 3 & 7.7 & $124.1-131.8$ \\
\hline & Upper & 150 & \pm 2 & 6.1 & $147.9-154.0$ \\
\hline \multirow{2}{*}{$\begin{array}{c}\text { Scatter } \\
(10 / 5 \mathrm{keV})\end{array}$} & Lower & 126 & \pm 4 & 10.0 & $121.9-131.9$ \\
\hline & Upper & 150 & \pm 2 & 6.1 & $147.9-154.0$ \\
\hline \multicolumn{2}{|c|}{$\begin{array}{l}167 \mathrm{keV} \text { Tl201 } \\
\text { primary peak }\end{array}$} & 167 & \pm 6 & 20.1 & $156.9-177.0$ \\
\hline
\end{tabular}


The primary T1201 counts in the Tc99m window can be estimated using the thallium peak at $167 \mathrm{keV}$. An initial estimate of how many counts reside in the $135 \mathrm{keV}$ will be produced from a modified ratio of abundances of the two secondary T1201 peaks. This estimate can then be subtracted accordingly. Recall the abundance of these two peaks being $2 \%$ for the $135 \mathrm{keV}$ peak and $8 \%$ for the 167 peak from section 1.4 .1 . A logical starting point for the scaling factor is $25 \%(2 / 8)$. However, subtraction of $25 \%$ of the counts from the $167 \mathrm{keV}$ peak resulted in overcorrection of the images. The exact cause of the overcorrection is unclear but may be partly due to the fact that not all counts from the $135 \mathrm{keV}$ window are detected in the $140+/-6 \% \mathrm{keV}$ primary $\mathrm{Tc} 99 \mathrm{~m}$ window. To avoid the overcorrection, the abundance of the $135 \mathrm{keV}$ peak was decreased in our scaling factors. Accuracy of the correction using scaling factors ranging from $1.25 \%$ $(0.1 / 8)$ to $9.37 \%(0.75 / 8)$ in increments of $0.625 \%(0.05 / 8)$ was evaluated.

\subsubsection{Phase 2 - Estimation of Tc99m cross-talk in the T1201 primary window.}

In the second phase of this study, TEW corrections will be applied to the T1201 primary window. The TEW method will first be evaluated for all 52 patients imaged with Tc99m. At about $30 \%$, the presence of cross-talk from Tc99m in the Tl201 window is severely abundant, as shown in table 2.2 . There is great need for accurate cross-talk correction in this window. 


\subsubsection{Energy windows considered}

To apply the TEW correction to the T1201 image, we require a number of different energy windows, including the window containing the T1201 primary peak and two scatter windows above and below this primary peak. The primary window of the T1201 window used clinically is an asymmetric window centered at $70 \mathrm{keV}$. The asymmetric-window is required to capture all the information from the collection of mercury $\mathrm{x}$-rays emitted by this isotope of thallium.

The same scatter window widths as were used for correcting the Tc99m window are again used in the T1201 window $(3,5,8$ and $10 \mathrm{keV})$, to try and find the optimal pairing of low noise and accurate correction. Due to limitations in Lister previously described combined with the lower energy of the central values, it was necessary to use asymmetrical windows for the scatter windows in order to get more exact values for the window widths.

The exact values of all window widths used for the TEW correction in the Tc99m primary window are listed in table 2.4 .

\subsubsection{Modifications to TEW}

The position of the primary T1201 window used clinically leads to complications that need to be avoided during the correction. First, the upper limit of the energy window lies at $86 \mathrm{keV}$. Should some of the gamma rays from either T1201 or Tc99m be absorbed by the collimator and produce characteristic tungsten $\mathrm{x}$-rays (the pinhole aperature in this 
collimator is made of tungsten), they will be registered directly in the upper scatter window, including unwanted counts in the scatter estimation. Some Tungsten characteristic $\mathrm{x}$-rays have energies of $57.0 \mathrm{keV}$ and $69.5 \mathrm{keV}$. (68) In figure 1.13 , we see a bump in the Tc99m scatter counts above the main T1201 peak. This bump could be caused by Tungsten $\mathrm{x}$-rays from interactions with the collimator or as backscatter or escape peaks. To avoid misestimating scatter by including this bump in the scatter estimate, the upper scatter window was shifted. Its lower energy boundary will be at 100 $\mathrm{keV}$, instead of being directly adjacent to the upper boundary of the primary energy window, $86 \mathrm{keV}$. In order to compensate for this shift, the counts directly above the primary T1201 window were interpolated on a pixel-by-pixel basis using the counts in both of the scatter windows accumulated. With this new information, a simple TEW subtraction can be used to remove any intermediate counts in our scatter estimate, to not over-estimate scatter in the primary image.

Upon closer examination of the $\mathrm{Tc} 99 \mathrm{~m}$ scatter profile in the $\mathrm{T} 1201$ primary window, a substantial drop in counts occurs just below the lower boundary of the T1201 primary window This drop does not affect the correction for narrower scatter windows (eg. $3 \mathrm{keV}$ ), but as the width of the lower scatter window grows, the accuracy of the scatter estimate will drop. This can be avoided by scaling the total counts in the wider scatter windows to match those in the $3 \mathrm{keV}$ window, correcting in the difference in window widths. A scaling factor for the counts in the lower scatter window can be determined by taking the total counts per $\mathrm{keV}$ in each of the lower scatter windows and fitting a second-order polynomial to the total counts versus window width curve. 
Table 2.4 Energy window information for TEW correction in T1201 primary window

\begin{tabular}{|c|c|c|c|c|c|}
\hline \multicolumn{2}{|c|}{ Energy Window } & $\begin{array}{c}\text { Center } \\
(\mathrm{keV})\end{array}$ & $\begin{array}{c}\text { Width } \\
(\%)\end{array}$ & $\begin{array}{l}\text { Width } \\
(\mathrm{keV})\end{array}$ & $\begin{array}{c}\text { Range } \\
(\mathrm{keV})\end{array}$ \\
\hline \multicolumn{2}{|c|}{ Primary } & 70 & $-14 /+23$ & 25.9 & $59.8-85.7$ \\
\hline \multirow{2}{*}{$\begin{array}{l}\text { Scatter } \\
(3 \mathrm{keV})\end{array}$} & Lower & $57^{\circ}$ & $-1 /+4$ & 2.9 & $57.7-60.6$ \\
\hline & Upper & 101 & $-1 /+2$ & 3.0 & $99.5-102.5$ \\
\hline \multirow{2}{*}{$\begin{array}{l}\text { Scatter } \\
(5 \mathrm{keV})\end{array}$} & Lower & 57 & $-5 /+4$ & 5.3 & $55.3-60.6$ \\
\hline & Upper & 101 & $-1 /+4$ & 5.0 & $100-5-105.5$ \\
\hline \multirow{2}{*}{$\begin{array}{l}\text { Scatter } \\
(8 \mathrm{keV})\end{array}$} & Lower & 57 & $-10 /+4$ & 8.1 & $52.2-60.3$ \\
\hline & Upper & 101 & $-1 /+7$ & 8.2 & $99.9-108.1$ \\
\hline \multirow{2}{*}{$\begin{array}{l}\text { Scatter } \\
(10 \mathrm{keV})\end{array}$} & Lower & 57 & $-13 /+4$ & 9.9 & $50.7-60.6$ \\
\hline & Upper & 101 & $-1 /+9$ & 10.1 & $99.9-110.0$ \\
\hline
\end{tabular}

\subsubsection{Single-sided TEW}

As can be seen in figure 1.14 (T1201 patient spectrum), there are a lot of counts in the Tl201 spectrum in the $50-60 \mathrm{keV}$ range of the T1201 primary window. These counts are due to the low energy tail of the CZT camera as well as photons that have scattered inside the patient.

These represent T1201 counts that are included in the cross-talk estimation, which should be mostly counts from Tc99m. The counts from the low energy tail of the CZT are 
primary counts that represent good spatial information that are included in the cross-talk estimate and will thus cause good primary counts to be removed from the Tl201 image.

A single sided TEW correction method was considered to avoid removal of all of these good counts. Both scatter windows were chosen above the primary Tl201 window, where there are minimal counts from Tl201 but still plenty of counts from Tc99m to create the cross-talk estimate. The estimate is produced with the assumption that the profile of the cross-talk counts has a consistent shape between the primary T1201 window and the scatter windows used for the single-sided correction. Assuming the cross-talk has the same slope, the number of counts due to cross-talk in the primary window can be extrapolated from the counts in the two scatter windows located above the primary T1201 window. The number of counts per pixel in the primary image due to cross-talk is estimated using the following equation:

$$
C_{c}=w_{p}\left[\frac{\left(\frac{C_{b}}{w_{b}}-\frac{C_{a}}{w_{a}}\right)}{\left(E_{b}-E_{a}\right)}\left(E_{p}-E_{b}\right)+\frac{C_{b}}{w_{b}}\right]
$$

where $C_{i}$ is the number of counts in the pixel, $w_{i}$ is the width of the window and $E_{i}$ is the center energy of the window. The subscript $i$ specifies the type of window with $a$ and $b$ representing the low and high scatter windows respectively, while $\mathrm{p}$ represents the primary T1201 window. The variable $C_{c}$ represents the number of counts from Tc99m cross-talk in the primary T1201 window. A schematic representation of this can be found in figure 2.1. 


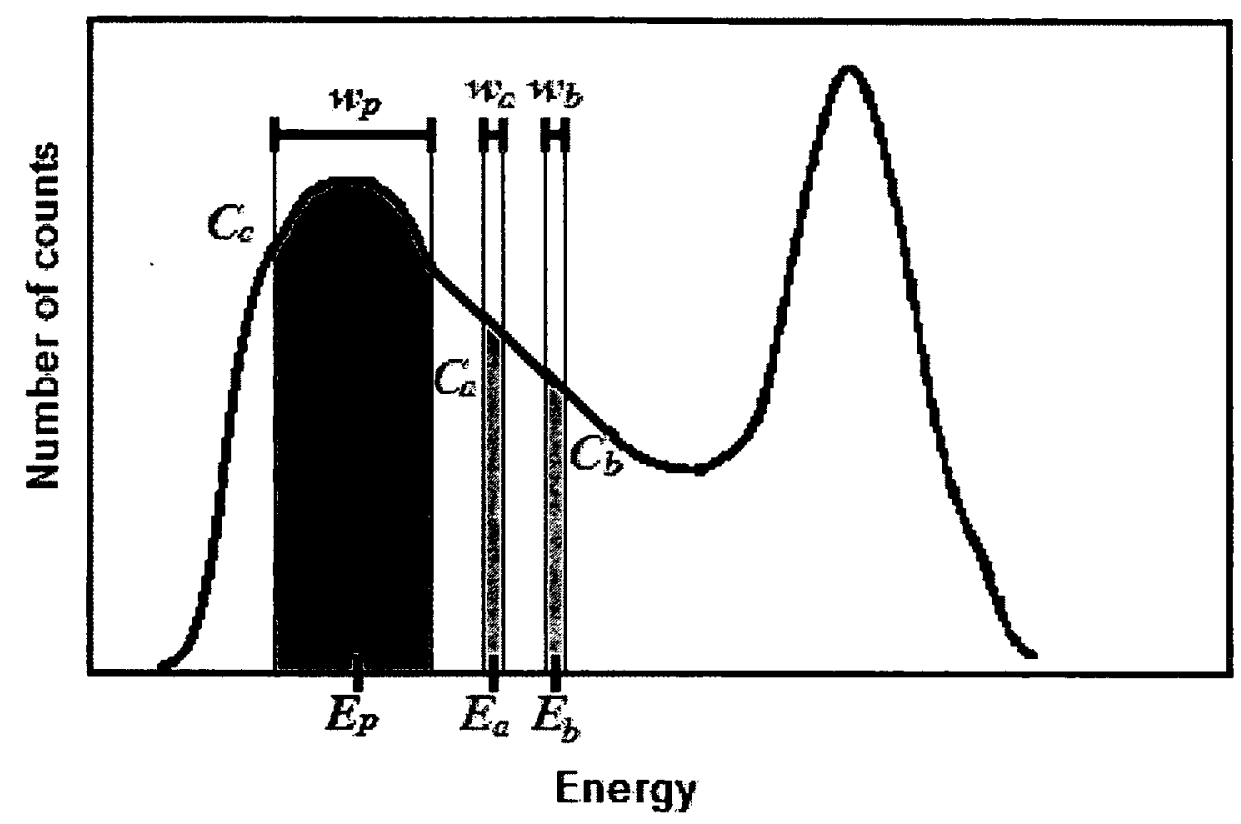

Figure 2.1 Schematic representation of the Tc99m energy spectrum showing the windows used for single sided cross-talk estimation of counts in the Tl201 primary photopeak window. $C_{a}$ and $C_{b}$ are the counts in lower and upper scatter windows respectively (of width $w_{a}$ and $w_{b}$ and central energy $E_{a}$ and $E_{b}$ ) while $C_{c}$ represents the number of counts estimated to be scatter in the primary window of width $w_{p}$ and central energy $E_{p}$.

Table 2.5 shows the energy windows used in order to create our scatter images. The correction is done in the same primary window. 
Table 2.5 Energy window information for single sided TEW correction in T1201 window

\begin{tabular}{|c|c|c|c|c|c|}
\hline \multicolumn{2}{|c|}{ Energy Window } & $\begin{array}{c}\text { Center } \\
(\mathrm{keV})\end{array}$ & $\begin{array}{c}\text { Width } \\
(\%)\end{array}$ & $\begin{array}{c}\text { Window } \\
(\mathbf{k e V})\end{array}$ & $\begin{array}{c}\text { Range } \\
(\mathrm{keV})\end{array}$ \\
\hline \multirow{2}{*}{$\begin{array}{c}\text { Scatter } \\
(3 \mathrm{keV})\end{array}$} & Lower & 96 & $-1 /+2$ & 2.8 & $94.6-97.4$ \\
\cline { 2 - 6 } & Upper & 106 & $-1 /+2$ & 3.2 & $104.4-107.6$ \\
\hline \multirow{2}{*}{$\begin{array}{c}\text { Scatter } \\
(\mathbf{5} \mathbf{~ k e V})\end{array}$} & Lower & 96 & $-1 /+4$ & 4.8 & $95.5-100.3$ \\
\cline { 2 - 6 } & Upper & 106 & $-1 /+4$ & 5.3 & $105.4-110.7$ \\
\hline \multirow{2}{*}{$\begin{array}{c}\text { Scatter } \\
(\mathbf{8} \mathbf{~ k e V})\end{array}$} & Lower & 96 & $-1 /+7$ & 7.7 & $95.0-102.7$ \\
\cline { 2 - 6 } & Upper & 114 & $-3 /+4$ & 7.9 & $110.0-117.9$ \\
\hline \multirow{2}{*}{$\begin{array}{c}\text { Scatter } \\
(\mathbf{1 0} \mathbf{~ k e V})\end{array}$} & Lower & 96 & $-1 /+9$ & 9.6 & $95.0-104.6$ \\
\cline { 2 - 6 } & Upper & 112 & $-1 /+8$ & 10.1 & $111.4-121.5$ \\
\hline
\end{tabular}

\subsubsection{Phase 3-Simulated dual-isotope corrections}

In the third phase of this study, the TEW correction with appropriate modifications from the previous two phases was applied. The correction was done in both the T1201 and Tc99m windows on the simulated dual-isotope images. 


\subsubsection{Analysis}

\subsubsection{Volume of Interest Selection}

Prior to analysis of results, a volume of interest (VOI) of the images was selected as a cube surrounding the heart. The heart was centered in the VOI, which contained only counts from the heart when possible. The volume was selected by close observation of the primary image along all three axes of the image $(x, y, z)$. Activity outside the heart should essentially be zero, which is hardly ever the case due to noise in the image. Limits for pixel values in $\mathrm{x}, \mathrm{y}$ and $\mathrm{z}$ were selected to be where the pixel value dropped to approximately $20 \%$ of the maximum pixel value in the image, using the color bar in the software to determine which pixels in the image are most likely only noise and not part of the heart volume. Figure 2.2 shows an example image of a slice of the heart before and after volume selection in MATLAB.

The reason for choosing a VOI around the heart for analysis is to eliminate activity from other organs picked up in the camera field of view (FOV) in the quantitative analysis performed. We see a clear example of this in figure 2.2 (a), where there is presence of high activity that is not part of the heart, as indicated by the white arrow. While the FOV of the camera used is limited and centered on the heart, the FOV does extend beyond the heart walls and other nearby organs can sometimes be seen. 

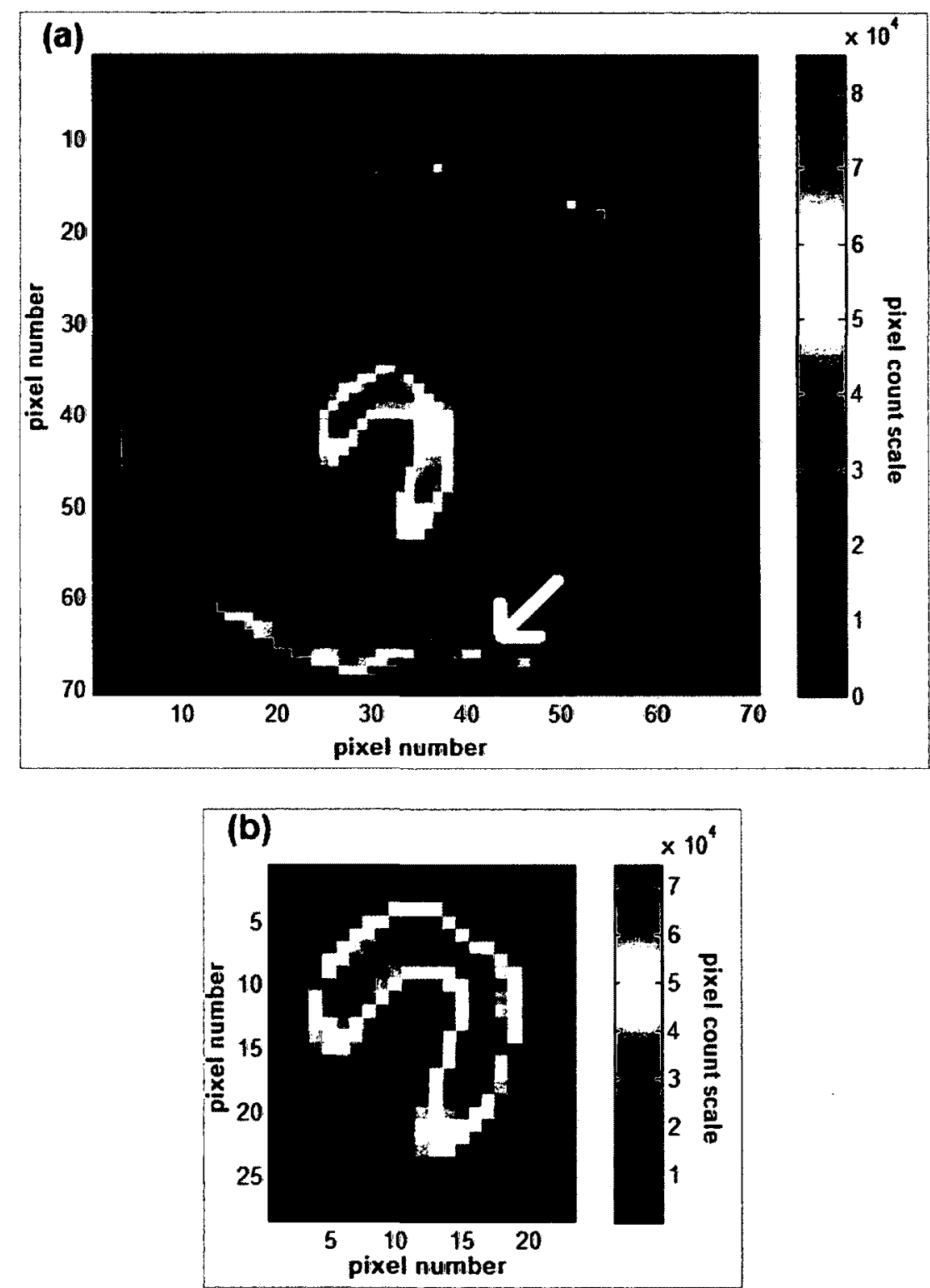

Figure 2.2 Example image showing a slice of the heart (a) before VOI selection and (b) after VOI selection. The white arrow in part (a) shows high activity from organs that are near the heart and should not be included in the qualitative analysis performed, justifying the need for a heart-only VOI as selected in part (b). 


\subsubsection{Single-Isotope Corrections}

For the single isotope studies (sections 2.2.5 and 2.2.6), after successful correction of the image, there should be no counts remaining in the images. Cross-talk estimates were assessed by taking the difference with the corresponding original image. All pixel counts in the difference image were normalized to the maximum pixel value in the uncorrected image, to eliminate variability in count levels between patients. The values are therefore expressed as a percentage of the maximum value in the uncorrected image.

The mean value and standard deviation of the pixels in the difference image were calculated for each patient. The mean value gives us an idea how well the correction worked, giving $0 \%$ for a perfect correction. The standard deviation of the pixel value gives us an idea on the level of noise introduced into the corrected images. Additional noise will drive up the pixel standard deviation of the difference image, as we will get more variability in the pixel values than if there were no noise. These values were averaged over the entire patient set, separated by gender. Average values of the standard deviation were plotted versus the mean pixel difference to assess correction bias and noise in the resulting images.

\subsubsection{Dual-Isotope Corrections}

After successful correction in dual-isotope studies (described in section 2.2.7), the image should be the same as our truth image in that same window (e.g. T1201 image in T1201 window). For corrections on dual-isotope contaminated images, corrected images were compared with the single-isotope uncontaminated images in the same window 
(truth). All images were normalized to the maximum value in the truth image to eliminate variability in count levels between patients. The mean and standard deviation of the pixel values in the image representing the difference in pixel values of the corrected image and the truth image were calculated and plotted the same way as described in section 2.2.9.2.

\subsubsection{Statistical Tests}

Results were compared to determine if there were significant differences among the different corrections. The mean bias and mean pixel standard deviation of the entire patient set (separated by gender) were calculated. Mean values were compared using a paired t-test. The t-statistic is calculated using equation 2-2.

$$
t=\frac{\left|\frac{1}{n} \sum_{i=1}^{n}\left(X_{1, i}-X_{2, i}\right)\right|}{\frac{S D_{\Delta}}{\sqrt{n}}}
$$

$X_{I, i}$ and $X_{2, i}$ are two paired measurements, $S D_{\Delta}$ is the standard deviation of the paired differences and $n$ is the number of paired measurements.

To determine significance of the differences between the data sets, we compare the t-statistic to a critical $t$-value. The critical $t$-value depends on the degrees of freedom of the data set and a pre-determined probability of erroneously identifying a difference as significant when it is not. (16) Critical t-values can be found from tables or with analysis tools available in Excel software. For our experiments, we have chosen a cut-off of $\alpha=0.05$ for significance, giving us a $5 \%$ probability of falsely identifying a difference. 


\subsubsection{Qualitative analysis}

The heart was divided into different sections using a 17 -segment model in order to assess perfusion. (2) The 17 segments are shown in figure 2.3 .

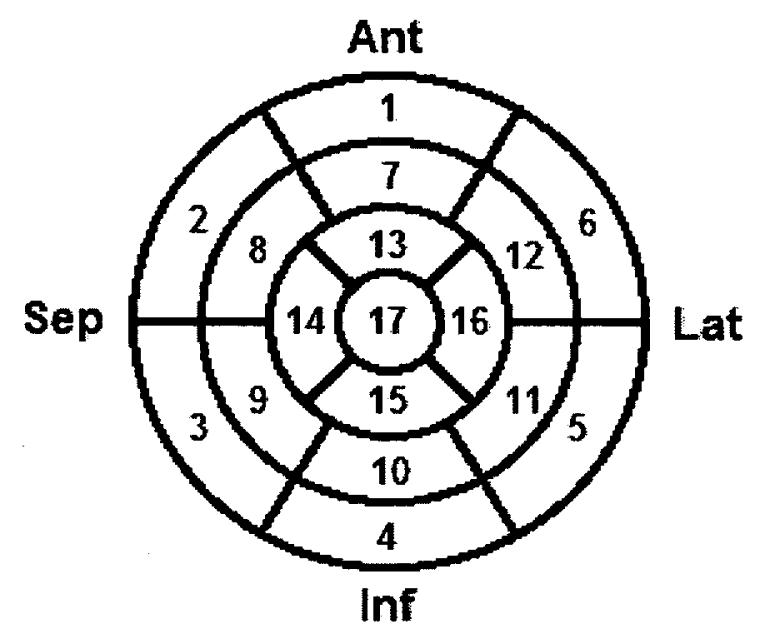

Figure 2.3 17-Segment Heart Model for Qualitative Analysis of Corrections. A perfusion score is assigned to each segment, expressed as a percentage of the maximum perfusion score. On this diagram Ant $=$ Anterior Wall, $\mathrm{Inf}=$ Inferior Wall, Sep $=$ Septum and Lat $=$ Lateral Wall.

A perfusion score is assigned to each of the 17 segments in the model shown above, to assess blood uptake in different parts of the heart (anterior wall (Ant), inferior wall (Inf), septum (Sep) and lateral wall (Lat). In this study, only visual comparisons were done between the dual-isotope contaminated image, the single-isotope truth image and various corrections to see if they work as we believe they will. 
In typical clinical MPI studies, each patient is assigned a Summed Stress Score (SSS) and a Summed Rest Score (SRS) in order to compare the heart in the two states and find any defects that may be present in the heart. Each segment is assigned a score $S_{i}$ between $0-4$, where 0 represents normal uptake, 1 represents a slight reduction in tracer uptake (mild), 2 represents a moderate reduction in uptake (moderate), 3 is a severe reduction in uptake (severe) and 4 is no uptake (absent).

\subsection{Results}

\subsubsection{Phase 1 - Estimation of T1201 counts in Tc99m primary window}

Quantitative results for the first part of the study are shown in figures 2.4, 2.5 and

2.6. Each of these figures presents two graphs, one for the male patient set and one for the female set. In all cases, the differences in the means of the two patient sets were statistically significant $(p<0.05)$. For the comparison of males to females, a $p$-value of $\mathrm{p}<0.05$ for the correction with $3 \mathrm{keV}$ scatter windows is found while $\mathrm{p}<0.03$ when using $10 \mathrm{keV}$ scatter windows.

In figure 2.4 , the data represent results from images corrected by TEW and by TEW with an added scaled subtraction of $25 \%$ of the $167 \mathrm{keV}$ T1201 peak. After scatter correction, there should be no signal from $\mathrm{Tl} 201$ in the $\mathrm{Tc} 99 \mathrm{~m}$ window, so the mean pixel value represents the mean bias in the correction. 

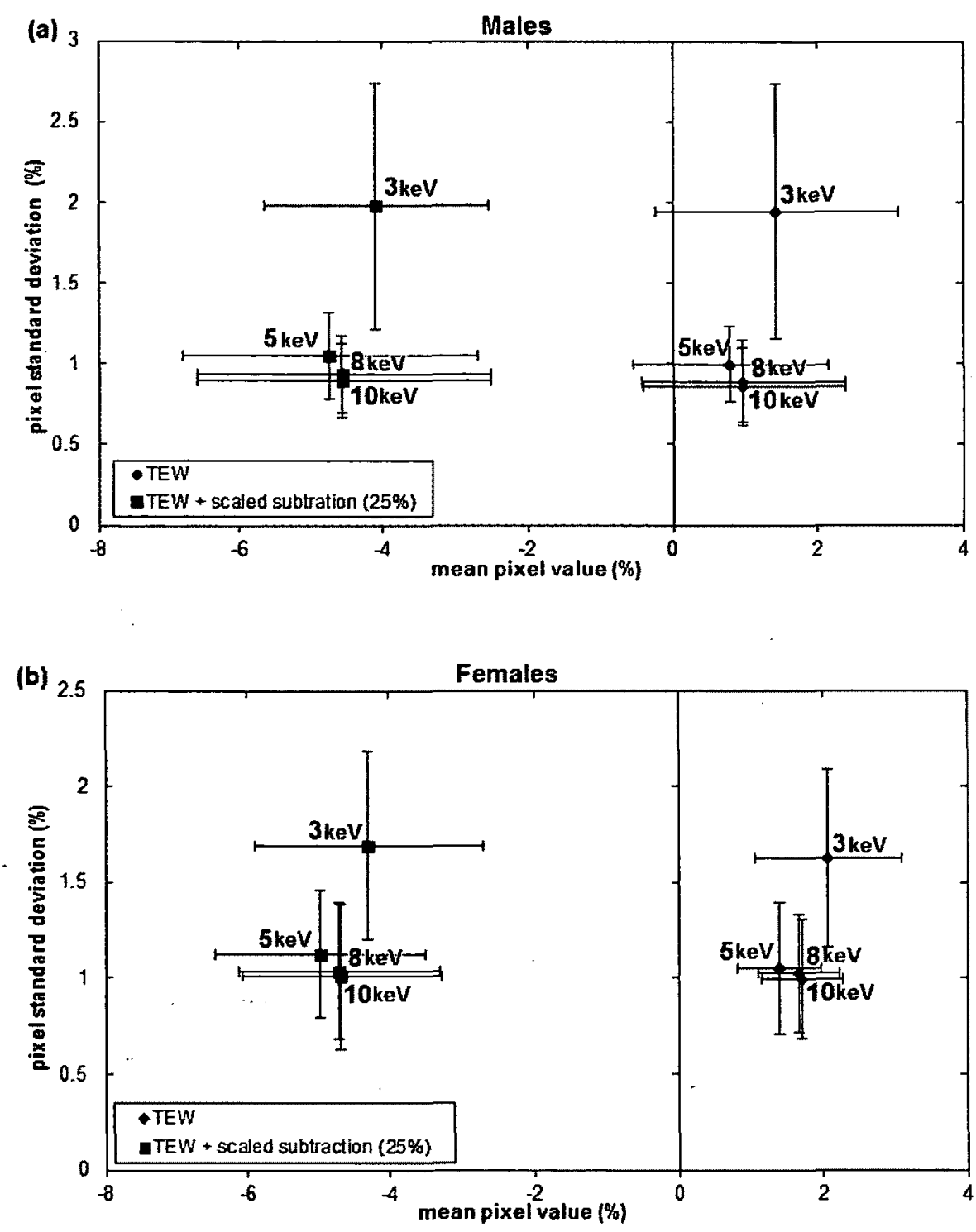

Figure 2.4 Pixel standard deviation vs. mean pixel value for the corrected image of T1201 counts in the Tc99m window. Each data point represents an average over all (a) 26 males and (b) 26 females in the study. Error bars represent the SD of the sample of (a) 26 males and (b) 26 females about the mean of the patient sample. 
In figure 2.4, the data represent results from images corrected with TEW and with TEW with an added scaled subtraction of $25 \%$ of the $167 \mathrm{keV}$ Tl201 peak. $25 \%$ was chosen to represent the ratio of emission abundances of the two peaks ( $2 \%$ for the 135 $\mathrm{keV}$ peak and $8 \%$ for the $167 \mathrm{keV}$ peak). There were significant differences in bias between the corrections with and without scaling for the same scatter window width $(p<0.05)$. There were no significant differences in SD between corrections with different scatter window widths $(p>0.2)$. The bias and standard deviation of images corrected with $3 \mathrm{keV}$ scatter windows were significantly different than bias and standard deviation of images corrected with wider scatter windows $(5,8$ and $10 \mathrm{keV})(\mathrm{p}<0.01)$. There were no significant differences in bias and standard deviation between scatter windows of wider widths for the same correction $(\mathrm{p}>0.1)$.

A wide range of scaling factors was explored and the scaling factor giving the closest bias to zero for each scatter window width was chosen to be applied in the dualisotope correction. These results are shown in figures 2.5 and 2.6. Figure 2.6 is simply a reproduction of figure 2.5 , taking a closer look around a mean pixel value of zero, to determine the ideal scaling factor over the entire patient set. The axes were limited between $-0.25 \%$ and $0.25 \%$. 

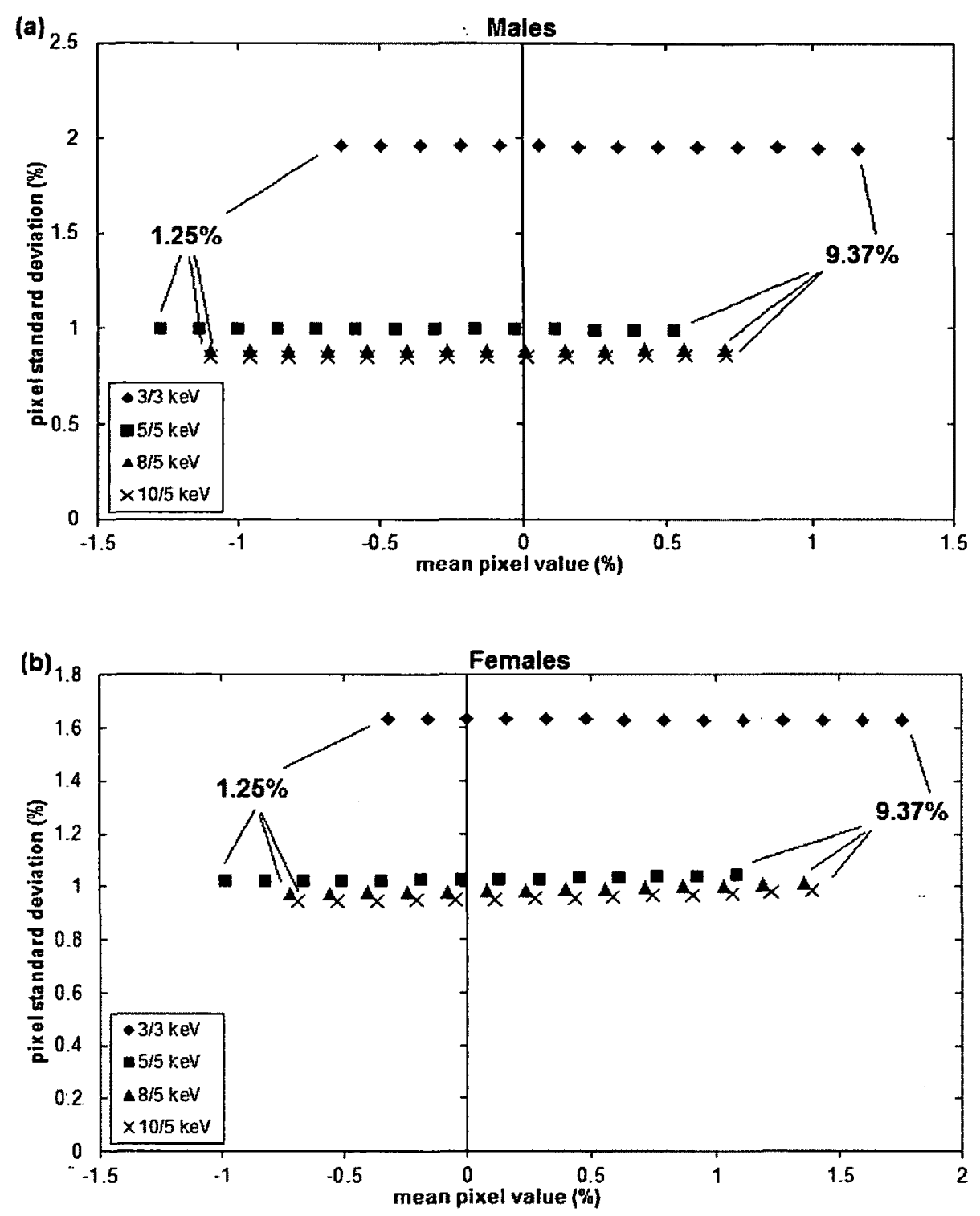

Figure 2.5 Pixel standard deviation vs. mean pixel value in corrected image of T1201 counts in Tc99m window. The correction applied was TEW with an added scaled subtraction of different percentages of the $167 \mathrm{keV}$ T1201 window, ranging from $1.25 \%$ to $9.37 \%$ in increments of $0.625 \%$. Each data point represented an average over all (a) 26 males and (b) 26 females in the study. 

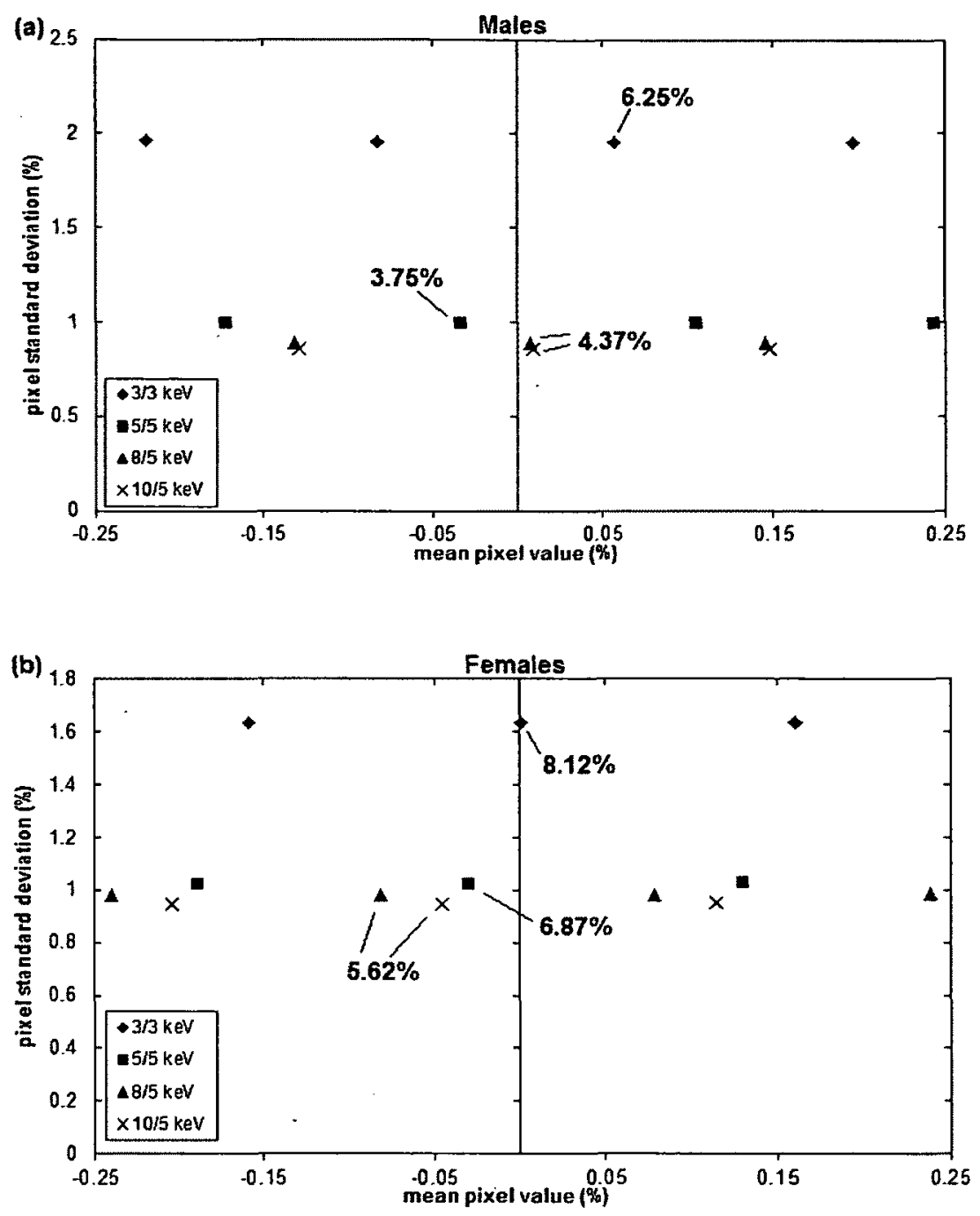

Figure 2.6 Pixel standard deviation vs. mean pixel value in corrected image of T1201 counts in Tc99m window. This figure is another representation of figure 2.5 , showing a smaller range of the mean difference $(-0.25 \%$ to $0.25 \%)$ Each data point represents an average over all (a) 26 males and (b) 26 females in the study. 
From figure 2.6, the scaling factors to be applied in the dual-isotope corrections were chosen. A different scaling factor is chosen for males and females. From these results, we can see that using wider scatter windows significantly improves the mean standard deviation of the pixel value throughout the patient set compared to using $3 \mathrm{keV}$ wide scatter windows $(p<0.005)$. However, there are no statistical differences between the bias of the corrected image using wider scatter windows $(5,8$ and $10 \mathrm{keV})(\mathrm{p}>0.2)$. Table 2.6 shows the scaling factors that were used in the dual-isotope corrections, determined from figure 2.6.

Table 2.6 Scaling factors applied to the $167 \mathrm{keV}$ T1201 peak to correct for the $135 \mathrm{keV}$ T1201 peak in the Tc99m window

\begin{tabular}{|c|c|c|}
\hline $\begin{array}{c}\text { Scatter Window } \\
\text { Width (keV) }\end{array}$ & \multicolumn{2}{|c|}{ Scaling Factor (\%) } \\
\cline { 2 - 3 } & Males & Females \\
\hline $\mathbf{3}$ & 6.25 & 8.12 \\
\hline $\mathbf{5}$ & 3.75 & 6.87 \\
\hline $\mathbf{8}$ & 4.37 & 5.62 \\
\hline $\mathbf{1 0}$ & 4.37 & 5.62 \\
\hline
\end{tabular}

From figure 2.6 , table 2.6 and the statistical tests previously described, the image corrected with scatter windows $10 \mathrm{keV}$ wide gives the correction with the lowest standard deviation in the pixel value while scaling factors of $4.37 \%$ and $5.62 \%$ for males and females respectively are required to get a better bias. 


\subsubsection{Dependence of scaling factor on BMI}

A study was done to determine if there is a correlation between the scaling factor that gives the best bias and the body-mass index (BMI) of the patient. The assessment was done with the corrections using $10 \mathrm{keV}$ scatter window width (as we see a better pixel standard deviation but no significant difference between results for different scatter window widths) and all scaling factors explored. Figure 2.7 shows a plot of the scaling factor that gives the ideal bias vs. BMI for both male and female patients.

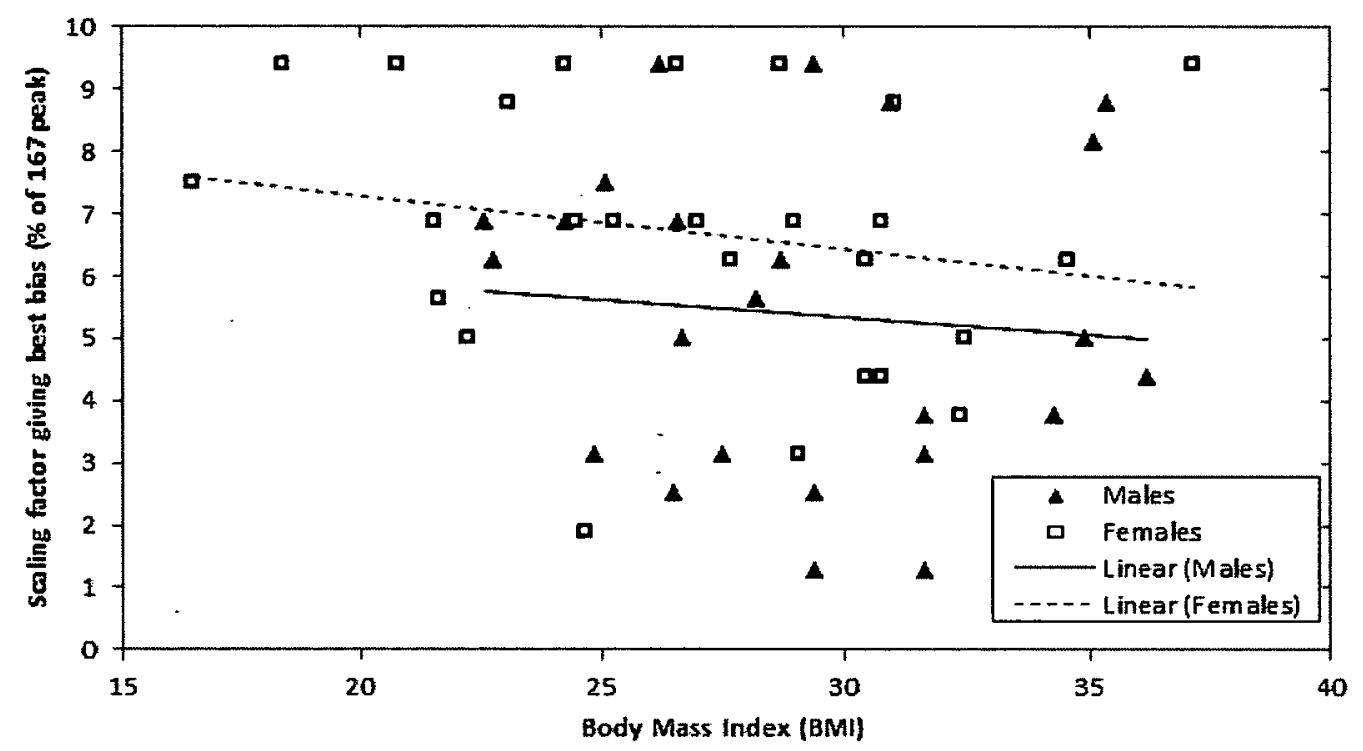

Figure 2.7 Scaling factor giving the best bias vs. BMI for both male and female patients. Male patients are represented as filled triangles while females are represented by squares. The graph also shows a linear trend line through both patient sets. 
There is evidently a wide spread in both patient sets shown in figure 2.7 . The calculated correlation coefficient between the ideal scaling factor and BMI of the patient is -0.0876 for the male patient set and -0.2021 for the female patient set. There is no significant correlation between the ideal scaling factor and BMI for males $(\mathrm{p}>0.6)$ or for females $(p>0.3)$.

\subsubsection{Phase 2 - Estimation of Tc99m cross-talk in the Tl201 primary window}

Quantitative results for this study are shown in figure 2.8. This figure presents two graphs, one for the male patient set and one for the female set. For all scatter window widths studied, the differences in the means of the two patient sets separated by gender were statically significant $(p<0.05)$.

In figure 2.8 , the data represent results from images corrected with TEW and with TEW with scatter windows scaled to the same count level as the $3 \mathrm{keV}$ scatter window.

We can see from figure 2.8 that applying only a TEW correction on Tc99m counts in the T1201 window provides a good correction for narrow scatter windows with increasing bias as the scatter windows get wider, there are significant differences in bias and standard deviation between corrections with wider scatter window widths $(5,8$ and 10 $\mathrm{keV})$ and corrections with $3 \mathrm{keV}$ scatter window widths. $(\mathrm{p}<0.05)$. There were no significant differences in bias or SD between the corrections with wider scatter window widths $(5,8$ and $10 \mathrm{keV})(\mathrm{p}>0.2)$. However, the wider scatter windows offer a lower 
standard deviation of the pixel values throughout the patient set. This is caused by the drop in counts in the $\mathrm{T} c 99 \mathrm{~m}$ spectrum at the lower boundary of the Tl201 primary window, as seen in figure 1.13.

By applying a scaling factor to the scatter images with wider scatter windows prior to correction in order to bring the counts back up to the same level as in the $3 \mathrm{keV}$ scatter windows, we see a significant improvement in the bias while keeping the lower standard deviation for 5, 8 and $10 \mathrm{keV}$ scatter window widths $(\mathrm{p}<0.05)$.

\subsubsection{Dual-isotope study}

Quantitative results for the dual isotope corrections are shown in figures 2.9 and 2.11. Each of these figures presents two graphs, one for the male patient set and one for the female set. For corrections applied with different widths of scatter windows in each primary window, the differences in the means of the two patient sets (males vs. females) were statistically significant $(p<0.02)$. and thus results should be separated by gender. This statement holds when scaling factors are applied as well. 

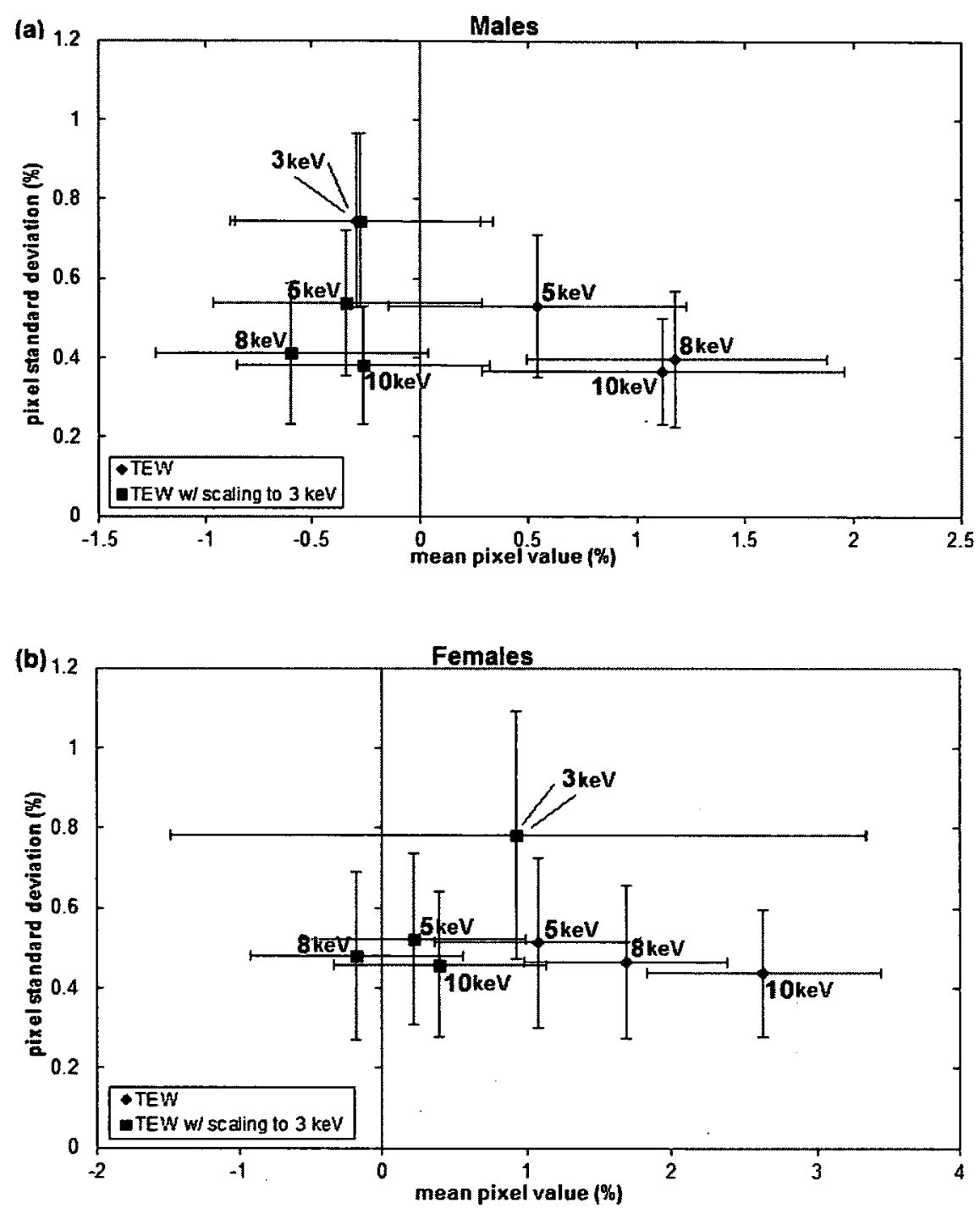

Figure 2.8 Pixel standard deviation vs. Mean pixel value in corrected image of Tc99m counts in T1201 window. TEW correction was applied as well as TEW while scaling the scatter windows to the same count level as the $3 \mathrm{keV}$ scatter window. Each data point represents an average over all (a) 26 males and (b) 26 females in the study. Error bars represent the SD of the sample of (a) 26 males and (b) 26 females about the mean of the patient sample. 
Figure 2.9 shows the results for the correction of the simulated dual-isotope study in the Tc99m window. The graph shows the results of the images corrected with only TEW as well as TEW with a scaled subtraction of the $167 \mathrm{keV}$ T1201 peak. The scaling factor for the scaled subtraction was determined from the single-isotope study (section 2.3.1).

We see from figure 2.9 that in dual-isotope corrections, the mean difference between the corrected and truth image is between $7 \%$ and $10 \%$ in all corrections explored for the corrections in the Tc99m window. The added scaled subtraction, which showed a $2 \%$ difference in mean counts for the single isotope study, shifts the mean difference in the dual-isotope study by $<1 \%$. There were no significant differences between the different scatter window widths with or without scaling (eg. $3 \mathrm{keV}$ with scaling vs. $5 \mathrm{keV}$ with scaling) ( $p>0.35)$. There were also no significant differences in bias and SD between the corrections with and without scaling for the same scatter window width (eg. $3 \mathrm{keV}$ with scaling vs. $3 \mathrm{keV}$ without scaling) $(\mathrm{p}>0.1)$.

The bias shown in figure 2.9 was between $7 \%$ and $10 \%$ for all corrections. However, in table 2.2, the average scatter fraction for T1201 counts in the Tc99m window is shown to be about $7 \%$ for males and $10 \%$ for females. There were no significant differences between the mean scatter fraction and the mean bias for each correction performed $(p>0.1)$. 

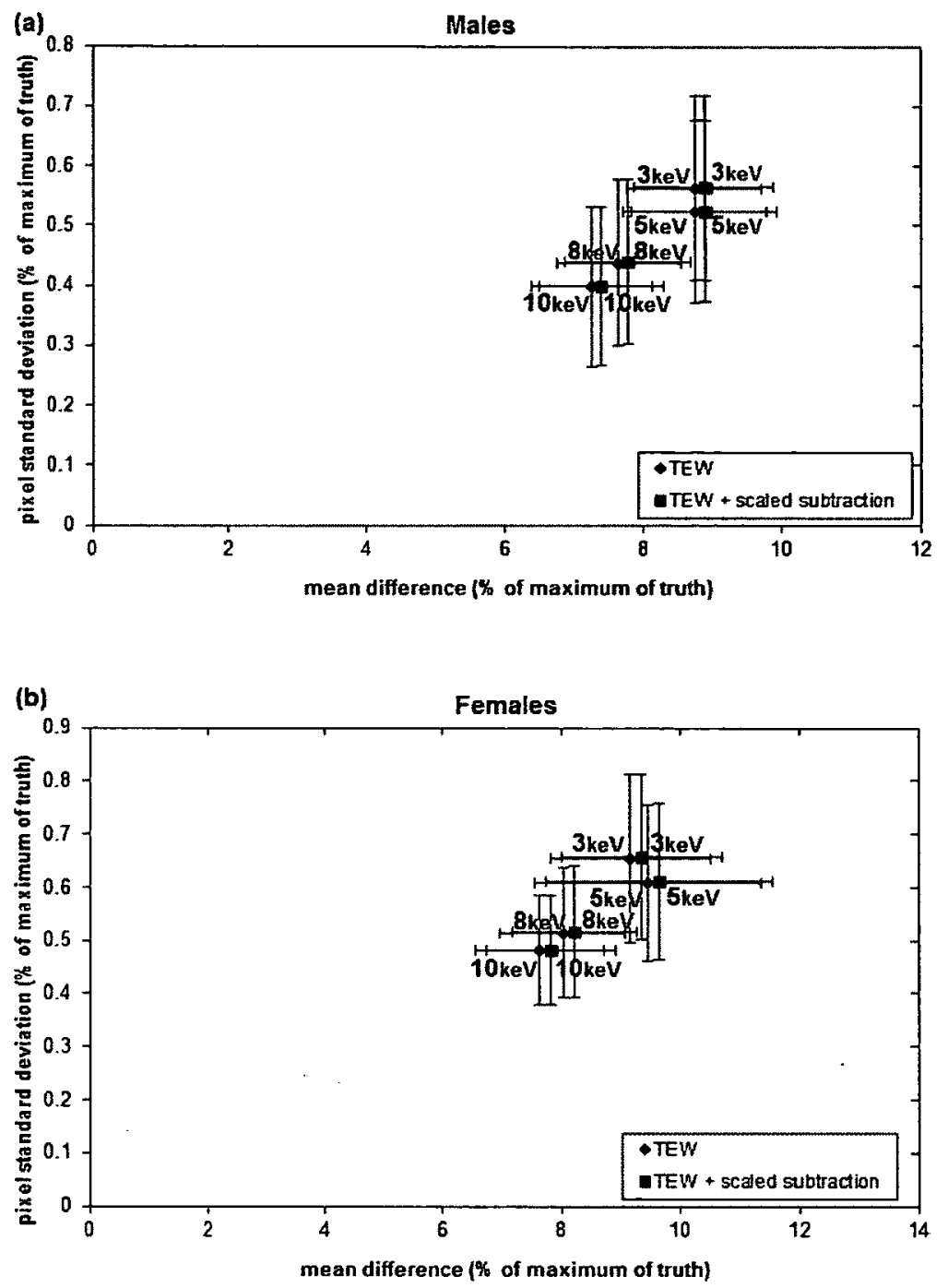

Figure 2.9 Pixel standard deviation vs. mean difference between the pixel values in the corrected contaminated image and the uncontaminated singleisotope image in $\mathrm{Tc} 99 \mathrm{~m}$ window. Corrections of TEW and TEW with an additional scaled subtraction of the $167 \mathrm{keV}$ T1201 peak were applied (for scaling factors of 167 peak, see table 2.6). Each data point represents an average over all (a) 26 males and (b) 26 females in the study. Error bars represent the SD about the mean of each sample. 
Figure 2.10 shows example images of the heart from the $\mathrm{Tc} 99 \mathrm{~m}$ window for a representative patient. In this image, we can see the truth image (a) (Tc99m single isotope image), the contaminated image (b) and two of the different corrections explored (c,d).

Figure 2.11 shows the results for the correction of the simulated dual-isotope study in the T1201 window. The graph shows the results of the images corrected with only TEW as well as TEW with the counts of the wider scatter windows $(5,8$ and 10 $\mathrm{keV}$ ) scaled to the count level of the $3 \mathrm{keV}$ window to account for the drop in counts in the $\mathrm{Tc} 99 \mathrm{~m}$ patient spectrum below the T1201 primary window. .

Figure 2.11 presents a mean difference between the corrected image and the truth image in the T1201 window between $15 \%$ and $20 \%$ for all corrections explored. These values present a significant improvement over the initial scatter fraction of $\mathrm{Tc} 99 \mathrm{~m}$ counts in the T1201 window of $30 \%$ for males and $25 \%$ for females $(p<0.01)$. However, while we saw an improvement in the mean pixel value in the single-isotope study while scaling the scatter windows to the $3 \mathrm{keV}$ count level in order to compensate for a drop in counts in the Tc99m spectrum at the lower edge of the T1201 window (see figure 1.13), we do not see this improvement in the dual-isotope case in any of the scatter window widths. Adding the counts from the T1201 spectrum affects the corrections applied to the Tc99m counts in this window. There is no significant difference in bias or SD between applying TEW with or without the additional scaling of the scatter windows ( $>0.1$ ). 


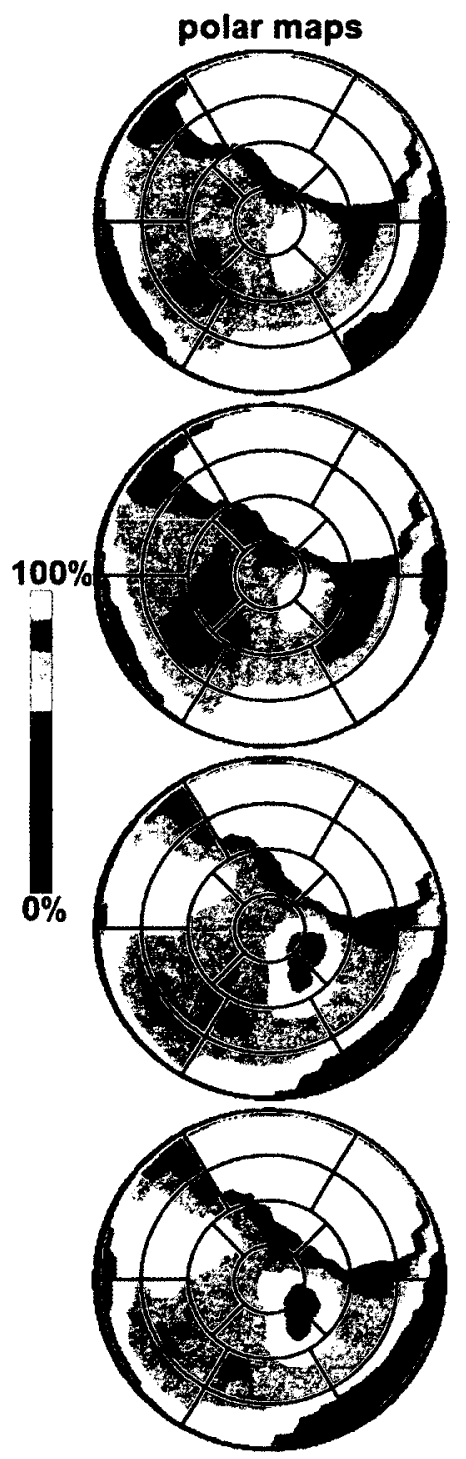

(a)
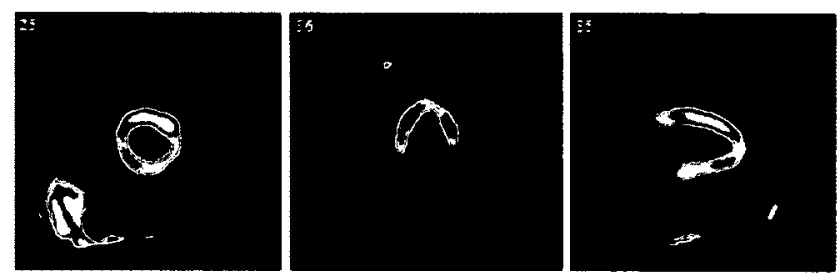

(b)
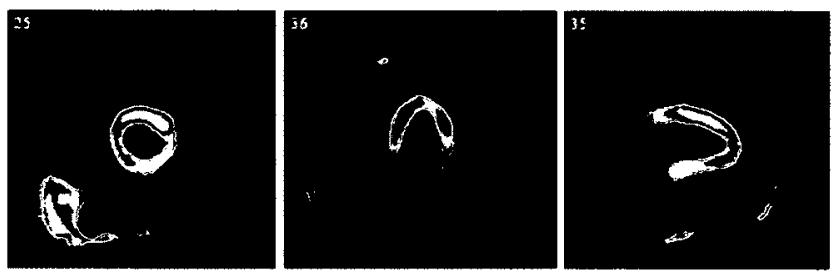

(c)
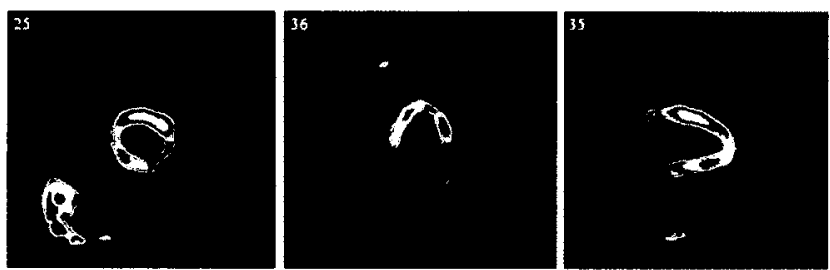

(d)
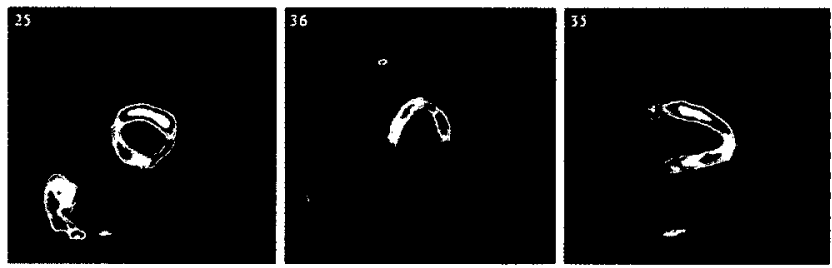

Figure 2.10 Example heart images (mid Short Axis (SA), Horizontal Long Axis (HLA) and Vertical Long Axis (VLA)) and polar maps in the Tc99m window.

(a) truth Tc99m image, (b) contaminated dual-isotope image, (c) TEW correction with $3 \mathrm{keV}$ scatter windows and (d) TEW correction with $3 \mathrm{keV}$ scatter windows with added scaled subtraction of the $167 \mathrm{keV}$ T1201 peak. 
Figure 2.12 shows example images of the heart from the T1201 window for a representative patient. In this figure, we show the truth image (T1201 single isotope image) (a), the contaminated image (b) and two of the different corrections explored $(\mathrm{c}, \mathrm{d})$.

Figure 2.12 shows an example of where cross-talk contamination in images introduces unwanted artifacts. Comparing the truth image to the dual-isotope contaminated image of figure 2.12 , it is clear that a defect is present in the dual-isotope SA and VLA image (lower portion of the heart, segment 10 of the polar map as given in key of figure 2.3) and not in the truth image, leading to a potential for misdiagnosis. However, this defect is still present in all of the corrected images, as well as new defects introduced by the corrections, most likely by having too many primary counts (from the low energy tail of the CZT for instance) in our scatter estimate.

We see from figure 2.12 that many unscattered counts are lost during the correction by comparing the truth image (a) with the different corrections $(c, d)$. In parts $c$ and $\mathrm{d}$ of figure 2.12 , many of the regions have reduced counts relative to the truth image. It can be hypothesized that too many primary (unscattered) counts are removed from the contaminated image during the correction. 

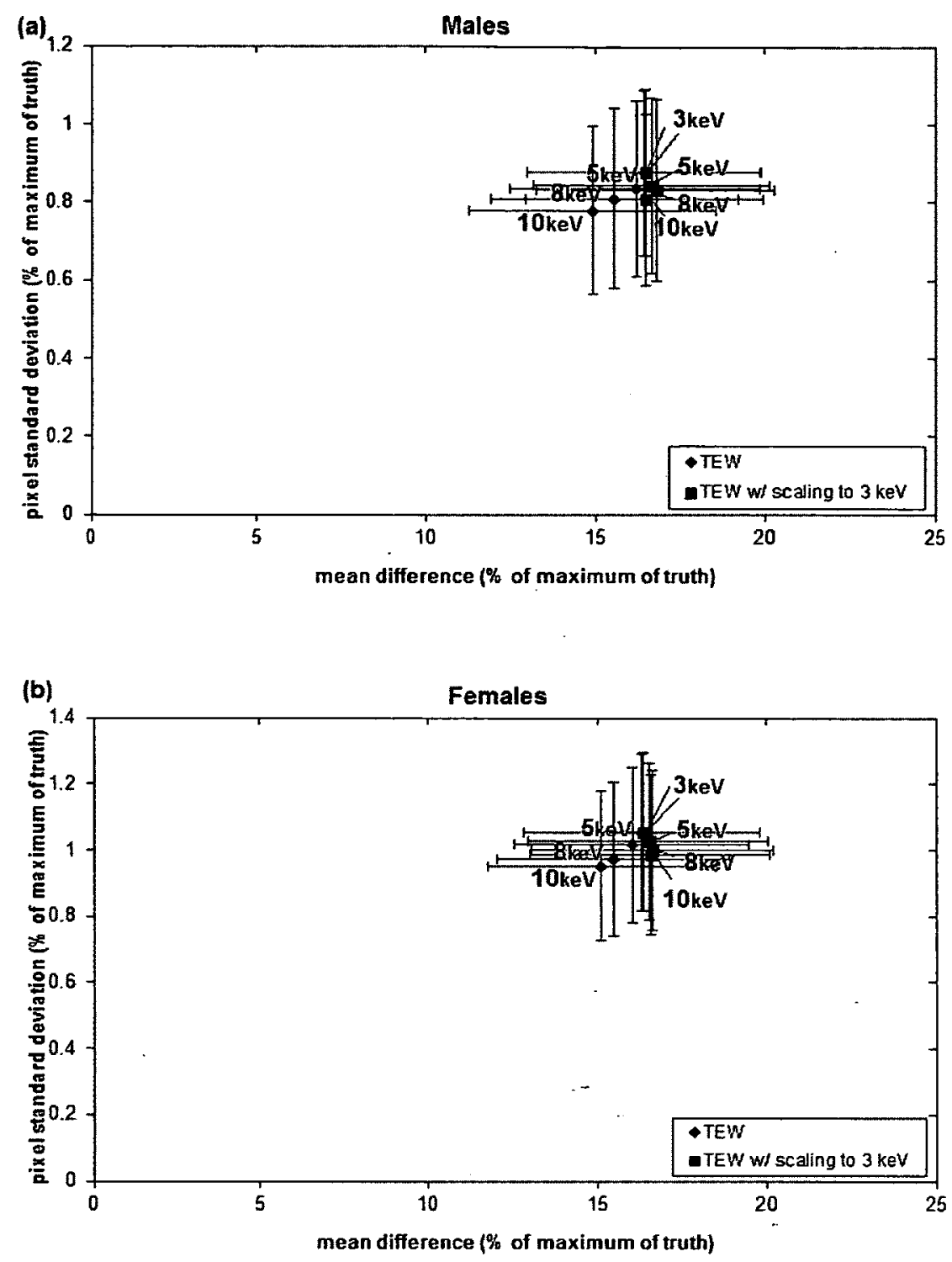

Figure 2.11 Pixel standard deviation vs. mean difference between the pixel values in the corrected contaminated image and the uncontaminated singleisotope image in corrected dual-isotope image in T1201 window. Each data point represents an average over all (a) 26 males and (b) 26 females in the study. Error bars represent the SD of the sample of (a) 26 males and (b) 26 females about the mean of the patient sample. 

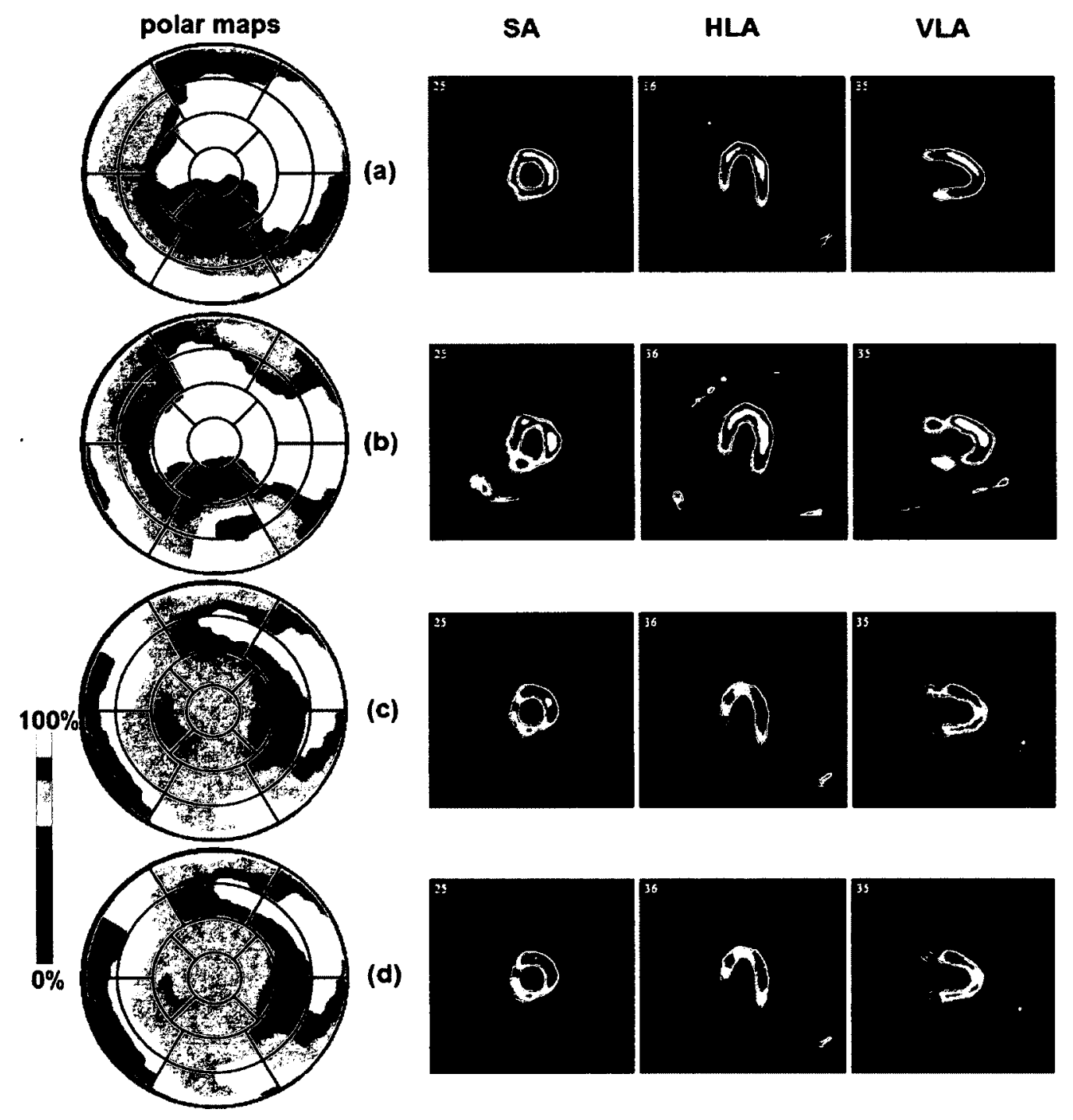

Figure 2.12 Example heart images (mid Short Axis (SA), Horizontal Long Axis (HLA) and Vertical Long Axis (VLA)) and polar maps in the T1201 window. (a) truth T1201 image, (b) contaminated dual-isotope image, (c) TEW with 3 $\mathrm{keV}$ scatter windows and (d) TEW w/ $10 \mathrm{keV}$ scatter windows with counts scaled to $3 \mathrm{keV}$ level. 
From figure 1.14, we see an abundance of T1201 counts in the region that would be used in the lower scatter window. These counts will affect our scatter estimation and remove many of the T1201 primary counts from our image, resulting in the degradation of image quality observed in figure 2.12. In order to compensate for this, a single-sided TEW method was developed, as described in section 2.2.6.3. The quantitative results of the single-isotope analysis for the single-sided TEW are shown in figure 2.13.

Figure 2.13 shows an overcorrection between $2 \%$ and $10 \%$ while using a singlesided TEW as described in section 2.2.6.3. The single-sided correction with $3 \mathrm{keV}$ scatter windows leads to a standard deviation of the normalized pixel values of $3 \%$. This is a significant increase in noise (standard deviation in the pixel value averaged over the entire patient set separated by gender) compared to TEW corrections with or without scaling $(p<0.01)$, where the average standard deviation of the pixel values in that difference images are both less than $1 \%$, as shown in figure 2.8 .

Figure 2.14 again shows a change in the results of the single sided corrections when including the T1201 counts. We now see a bias between $2 \%$ and $5 \%$ with no significant differences between corrections with different scatter window widths $(p>0.3)$. There were also no significant differences in SD between corrections $(p>0.1)$. 

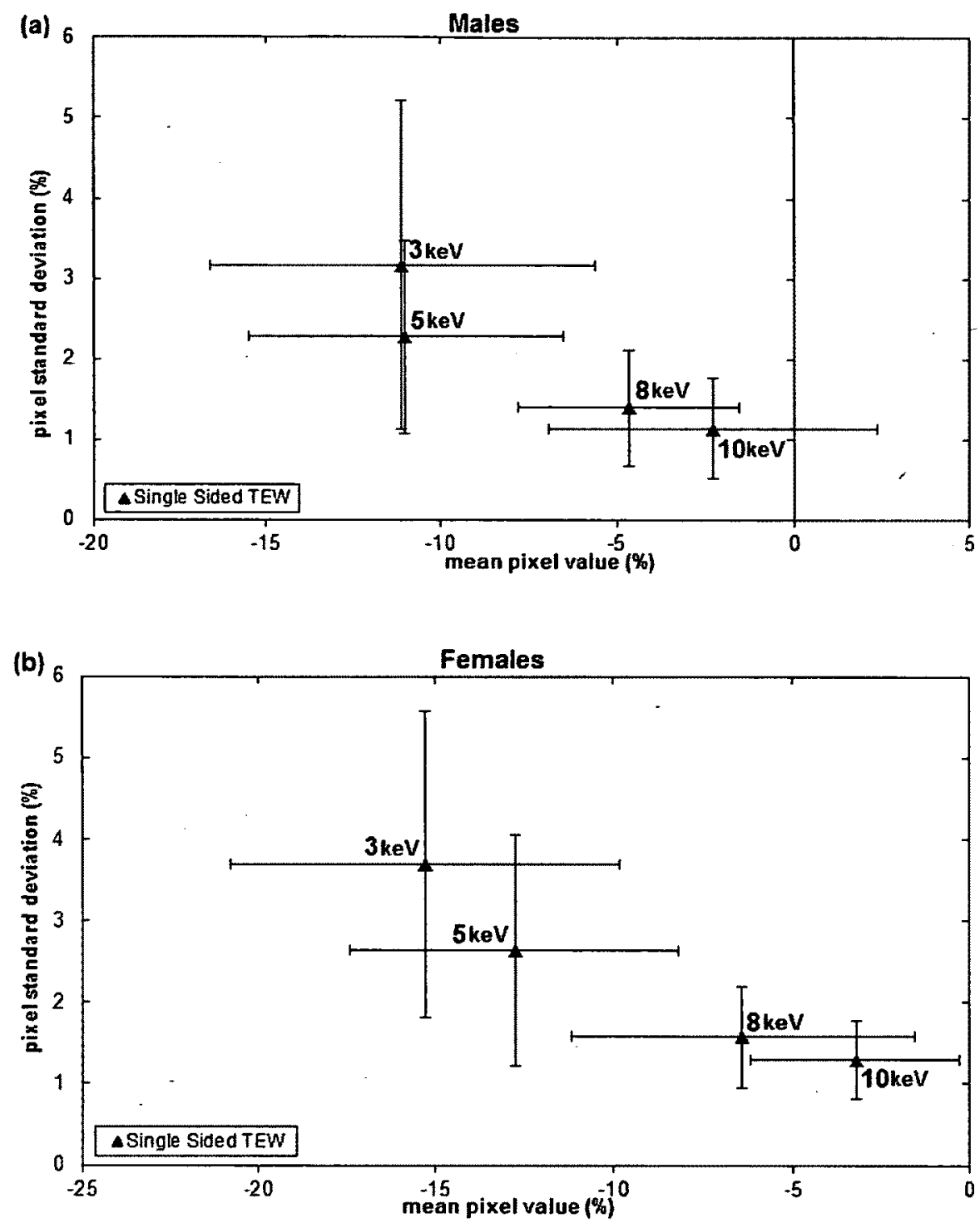

Figure 2.13 Pixel standard deviation vs. mean pixel value in corrected image of Tc99m counts in T1201 window (single isotope image). Single-sided TEW correction was applied Each data point represents an average over all (a) 26 males and (b) 26 females in the study. Error bars represent the SD of the sample of (a) 26 males and (b) 26 females about the mean of the patient sample. 
Figure 2.15 shows example images of the heart from the T1201 window for a representative patient. In this image, we can see the truth image (Tl201 single isotope image) (a), the contaminated image (b) and several of the different corrections explored (c-f). Parts (a) through (d) are reproduced from figure 2.12 (a-d) for easy comparison with images corrected with single sided TEW.

\subsection{Discussion}

In this thesis, we investigated cross-talk correction using a TEW estimate of cross-talk in dual-isotope SPECT imaging on a dedicated cardiac CZT camera. Modified TEW estimation methods were developed and an assessment of their accuracy was performed.

In single-isotope studies, the scaling factor used for the correction of T1201 counts in the $\mathrm{Tc} 99 \mathrm{~m}$ window was seen to depend on the gender of the patient (Figures 2.4, 2.5 and 2.6, table 2.6). Figure 2.4 shows significant differences between the bias achieved after correction between males and females when the images are corrected with just TEW. For the comparison of males to females, a $p$-value of $p<0.05$ for the correction with $3 \mathrm{keV}$ scatter windows was found while $\mathrm{p}<0.03$ when using $10 \mathrm{keV}$ scatter windows.

Females generally have a lower body mass index (BMI) then males, potentially resulting in different magnitude of scatter and cross-talk. We measured the correlation between BMI and the ideal scaling factor as discussed in section 2.3.1.1. However, the results showed no significant correlation between BMI and scaling factor $(p>0.3)$. 

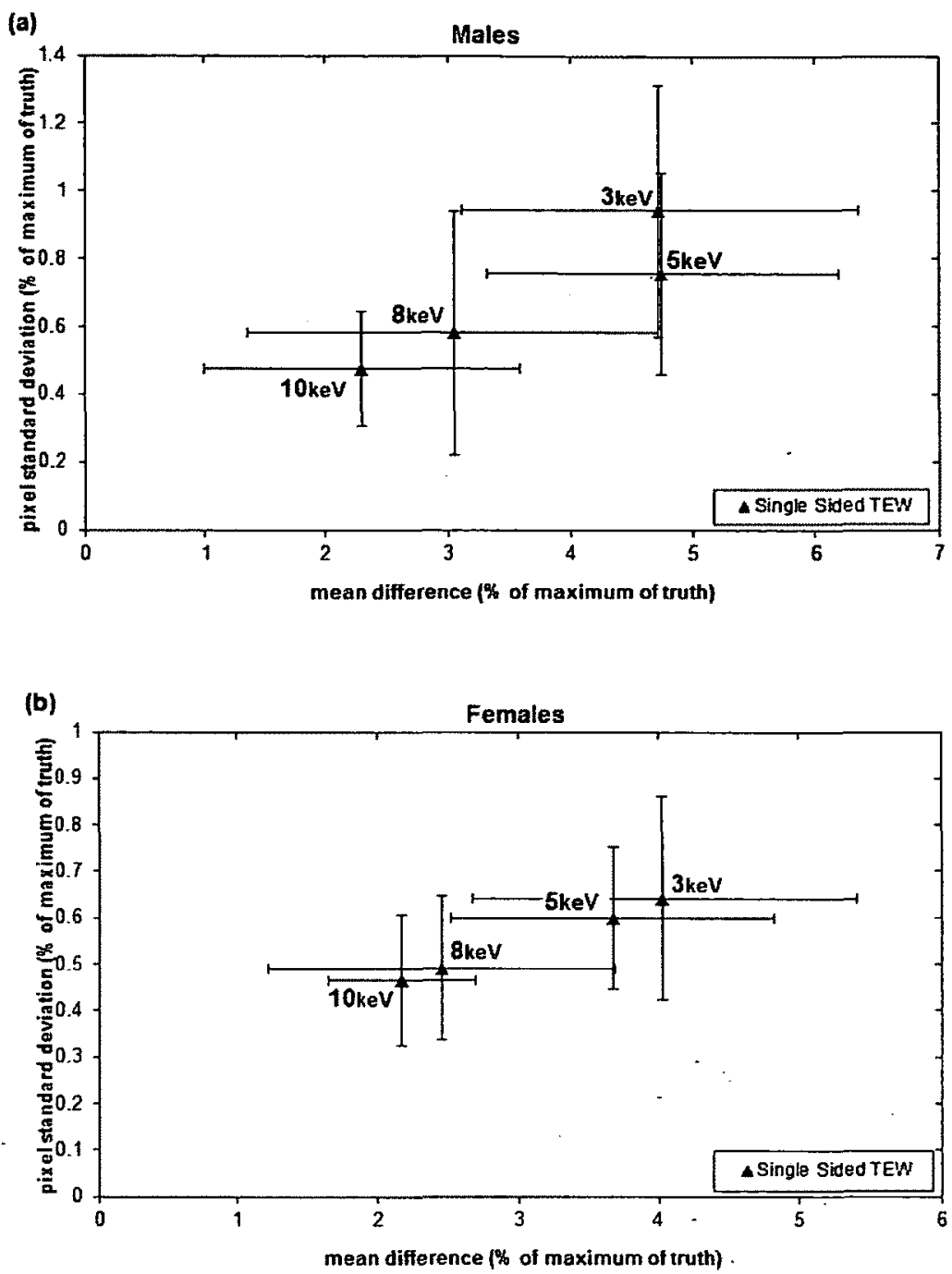

Figure 2.14 Pixel standard deviation vs. mean difference between the pixel values in the corrected contaminated image and the uncontaminated singleisotope image in corrected dual-isotope image in T1201 window. The data shown were corrected using the single-sided TEW method described previously. Each data point represents an average over all (a) 26 males and (b) 26 females in the study. Error bars represent the SD about the mean of the patient samples. 


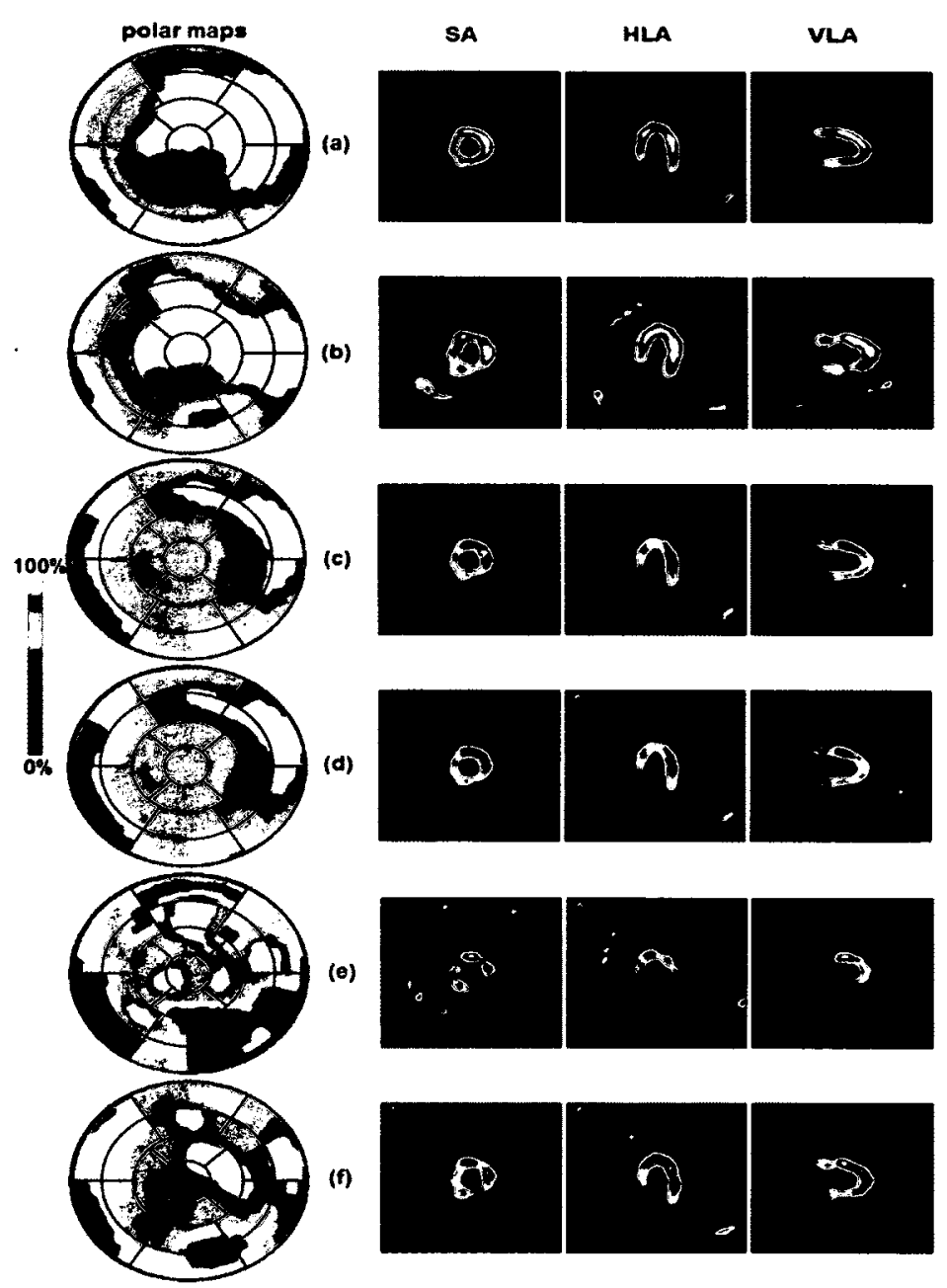

Figure 2.15 Example heart images (mid Short Axis (SA), Horizontal Long Axis (HLA) and Vertical Long Axis (VLA)) and polar maps in the T1201 window. (a) truth T1201 image, (b) contaminated dual-isotope image, (c) TEW with $3 \mathrm{keV}$ scatter windows and (d) TEW with $10 \mathrm{keV}$ scatter windows with counts scaled to $3 \mathrm{keV}$ level. Images with single sided TEW applied: (e) with $3 \mathrm{keV}$ scatter windows and (f) with $10 \mathrm{keV}$ scatter windows. 
In hindsight, this is not surprising. The scaling factor was used to remove primary T1201 counts from the Tc99m image, not scattered counts. BMI would differentially attenuate 167 and $135 \mathrm{keV}$ photons, but that effect should be minimal. Having ruled out correlation with BMI, the reason behind the significant gender differentiation seen in the scaling factor has yet to be determined and will be a point to explore in future studies.

Quantitatively, applying a single-sided correction in the T1201 window showed a significant improvement over classic two-sided TEW $(\mathrm{p}<0.01)$. For TEW corrections on simulated dual-isotope images in the T1201 window, the result showing the lowest mean and standard deviation (with $10 \mathrm{keV}$ scatter windows) still had a bias of $17 \%$. With a single-sided TEW correction using $10 \mathrm{keV}$ scatter windows, the bias was reduced to about $2 \%$. Unlike the other corrections applied in this study, there were significant differences in the scatter windows used $(p<0.01)$. However, single-sided TEW drives the noise level in the corrected images up. This is probably due to the fact that instead of interpolating the counts between the scatter windows (a bounded situation) we were extrapolating the counts to outside the range of our scatter windows (an unbounded situation). The scatter images were very noisy to begin with (even in $10 \mathrm{keV}$ windows), leading to misestimating the counts at every step of the correction and propagating this noise into our scatter estimate. This may be partly due to artifacts in the Tc99m scatter spectrum. We would not expect the scattered counts to rise at about $80 \mathrm{keV}$ as they do, since it does not follow the typical distribution of Compton scatter. The rise in counts could be partly due to characteristic tungsten x-rays, escape peaks or backscatter. 
The use of two different patients in our simulated dual-isotope studies could arguably have affected our data. Having not been imaged simultaneously means a misregistration of the rest/stress images, which may have led to changes in the reconstructed image. In certain cases, the hearts of the matched patients were also visibly different in size. The reconstruction is non-linear and misalignment of the projection data could affect the resulting image. However, in terms of the correction itself, this is not really an issue, as the goal was to add some sort of contamination to evaluate our corrections. The misalignment of the images simply detracts from the realism of the study.

All corrections in this study were done in image space. All images (both primary and scatter windows) were reconstructed prior to correction. Some of the images we worked with had very few counts, such as the $3 \mathrm{keV}$ scatter windows. Reconstructing images with very little counts can lead to big increases in noise levels. When dealing with the scatter windows, if we start with a noisy image, we will end up with a noisy estimate for cross-talk, leading to inaccurate corrections.

Two things will be considered in future work to try and avoid the noise propagation in our corrections from reconstructing poor counts. First, we will try smoothing the scatter images prior to calculating cross-talk estimation. Second, to avoid the problem of reconstructing low count images, the cross-talk estimation and correction will be performed in projection space (before reconstruction). By correcting in projection space, we eliminate the need for reconstruction of the images of our scatter windows, 
which have very poor count statistics. Reconstruction of the scatter windows lead to a great amount of noise in the images that were used for the correction.

Images from figures $2.10,2.12$ and 2.15 show some noise, even with the low values of standard deviation shown in the corresponding graphs showing bias and standard deviation. This may be caused in part by including a lot of voxels with low counts in the calculation of the standard deviation, where there was no activity but it is still part of the VOI. These pixels are not supposed to have many counts in them, representing the "hole" seen in the SA as well as corner pixels that have very few counts but must be there as we take a cubic VOI. A better method should be developed in order to estimate noise, perhaps along the lines of determining a VOI to exclude those low counts pixels, or using a method to have a heavier weighting of pixels with actual uptake from the heart in the standard deviation.

The use of CZT detectors in this study leads to complications that have not been faced with standard SPECT technology. A major factor to be considered is the presence of the low-energy tail of the CZT detector (section 1.5.3.1). Primary counts are present in all cross-talk estimations because of this, leading to a number of good primary counts being removed from our primary image. These counts should be accounted for in our correction. Methods to do so will be investigated as future work in this study.

Also having an effect on our corrections is the presence of Tungsten characteristic $\mathrm{x}$-rays from the pinhole aperture. Recall that Tungsten has characteristic $\mathrm{x}$-rays at 57.0 $\mathrm{keV}(67)$, which is in our lower scatter windows for corrections in the thallium window. 
This may also introduce primary counts into our scatter estimation. Limitations with Lister while creating our scatter images might also be an issue. From tables $2.3-2.5$, we see that for certain scatter windows, there is a bit of an overlap between the scatter window and the primary window (no more than $0.3 \mathrm{keV}$ ). Some of our scatter windows will therefore have some primary counts (on top of those provided by the low-energy tail of the CZT). However, especially at $140 \mathrm{keV}$, we are very limited by working with integer values in choosing boundaries of energy windows as a percentage of the center. There were no significant differences between different scatter window widths for the same TEW correction ( $p>0.5$ ), so this probably was not much of an issue.

In the Tc99m window, cross-talk presence is not as much of an issue as it is in the T1201 window. With such little impact on the quantitative results (from figure 2.9) and because it is very hard to see any differences in the heart images, full clinical evaluation of resting images should be performed in order to determine if the correction is even necessary.

Likewise, once a cross-talk correction for the T1201 image is developed, it too will need clinical evaluation. Clinical evaluation of the studies is required in order to assess clinical validity of the corrections. An initial evaluation can be done by comparing the summed perfusion scores of corrected images to the true values. Further evaluation would require reading of corrected and uncorrected studies by trained nuclear cardiology or nuclear medicine physicians. 
Upon comparison of figures 2.10 and 2.12 , we notice counts from the liver present in the Tc99m images (lower left-hand corner) but not in the Tl201 images. These counts also show up in the dual-isotope image in the Tl201 window (Figure 2.12b), while clearly being Tc99m. The correction removed most of these counts from the T1201 image (Figure $2.12 \mathrm{c}-\mathrm{f}$ ), removing on average $95 \%$ of scatter in each pixel of that VOI. This allows us to conclude that the modified TEW corrections applied in the T1201 window do remove Tc99m cross-talk from this window.

The results presented in this thesis demonstrated that the current modifications to the triple energy window cross-talk estimation, while correcting for cross-talk in primary energy windows, removed too many primary counts from the image and provided misleading images for clinical evaluation. Additional work is still needed to further improve this energy-window based approach to cross-talk estimation and correction. 


\section{Chapter 3: Conclusions and Future Directions}

Dual-isotope imaging is a potential strength for SPECT cardiac imaging and the new dedicated cardiac cameras offer potential advantages for its application. However, the design of these cameras is very different from traditional systems and this thesis has shown that simple application of a standard method for estimating cross-talk does not provide acceptable images. The TEW estimation $\cdot$ method, while having success in previous work with traditional NaI-based Anger-style cameras, leads to large increases in noise in the images simulated from the Discovery NM 530c dedicated cardiac camera. Further modifications will be needed in order to provide accurate cross-talk estimates in both the T1201 and Tc99m primary windows explored during this thesis.

This work has led to many interesting questions and suggested directions for further investigation. The single-isotope studies showed a statistically significant gender 
dependence on the scaling factor used to scale the $167 \mathrm{keV}$ Tl201 peak as an estimate for the $135 \mathrm{keV}$ peak. Further investigation is required in order to determine what could cause such differences.

The cross-talk fraction of T1201 counts into the Tc99m window was found to be $10 \%$. The clinical impact of this level of cross-talk on a resting $\mathrm{Tc} 99 \mathrm{~m}$ scan is unclear. It might be worth assessing this to determine the need for correction of the T1201 cross-talk. It might also be useful to explore different ways of quantifying noise levels in the corrected images. The method used in this thesis did not result in an accurate measure of the effect on the noise levels of the final corrected images. We suspect that this is due to having too many low counts in the VOI chosen for taking the standard deviation measure. A large number of low pixel values can give misleadingly low average values of noise estimation that are not necessarily reflected in the actual images provided by the correction.

Working in image space offers some advantages over working in projection space, but the proposed corrections can be applied in both domains. One drawback to working in image space is the reconstruction of images with very low counts. We are basically reconstructing an image that is mostly comprised of noise. Using these noisy scatter images to estimate corrections may drive up noise levels in the corrected image. We might first want to explore smoothing our reconstructed scatter-window images before determination of scatter estimates for correction. Another approach would be to attempt the correction in projection space (prior to reconstruction). 
Another approach that might be interesting is to attempt to use a different kind of energy window based correction. The need for cross-talk correction in simultaneous $\mathrm{Tl} 201 / \mathrm{Tc} 99 \mathrm{~m}$ SPECT is to compensate for cross-talk from Tc99m in the Tl201 window. Throughout the entire patient set (males and females), we have noticed that the scatter profile as seen in figure 1.13 remains roughly the same shape and is scaled to a certain count level that differs from one patient to another. Describing the profile of the spectrum and using an energy window between the primary peaks of the two isotopes, where there are few counts from T1201, to scale the cross-talk profile in the Tl201 window could be a simple way to estimate the scatter. Exploring methods to estimate the contribution of the low-energy tail in order to remove these counts from the scatter windows might also be an interesting path to follow.

In conclusion, the results presented in this thesis show that image-based TEW alone is not sufficient in order to compensate for cross-talk in Tc99m/Tl201 dual-isotope MPI on a dedicated cardiac camera with CZT detectors. The modifications proposed showed slight quantitative improvements but we hypothesize that too many primary counts are being included in all scatter estimates. Too many perfectly good counts are being removed from the image providing images that do not reflect the true state of the heart and that may lead to misdiagnosis, false normal or even unnecessary invasive procedures for the patient. However, there are still many roads to explore with cross-talk corrections for these specific isotopes which could lead to a simple but successful implementation of simultaneous $\mathrm{Tc} 99 \mathrm{~m} / \mathrm{Tl} 201$ dual-isotope SPECT imaging on a dedicated cardiac CZT camera. 


\section{References}

(1) Statistics Canada. Mortality, Summary List of Causes 2008. Released October 18, 2011.

(2) Hansen CL et al. American Society of Nuclear Cardiology Imaging Guidelines for Nuclear Cardiology Procedures. Journal of Nuclear Cardiology 14-6 e39 - e60 (2006)

(3) Ben-Haim S, Kacperski K, Hain S, Van Gramberg D, Hutton BF, Erlandsson K, Sharir T, Roth N, Waddington WA, Berman DS, Ell PJ. Simultaneous Dual-Radionuclide Myocardial Perfusion Imaging with a Solid-State Dedicated Cardiac Camera. European Journal of Nuclear Medicine and Molecular Imaging 37 1710-1721 (2010)

(4) Kiat H, Germano G, Friedman J, Van Train K, Silagan G, Wang FP, Maddahi J, Berman D. Comparitive Feasibility of Separate or Simultaneous Rest Thallium/Stress Technetium-99m-Sestamibi Dual-Isotope Myocardial Perfusion SPECT. Journal of Nuclear Medicine 35-4 542-548 (1994)

(5) Knešaurek K, Machac J. Comparison of Correction Techniques for Simultaneous ${ }^{201} \mathrm{~T} 1{ }^{99 \mathrm{~m}} \mathrm{Tc}$ Myocardial Perfusion SPECT Imaging: A Dog Study. Physics in Medicine and Biology 45 N167-N176 (2000)

(6) Lowe VJ, Greer KL, Hanson MW, Jaszczak RJ, Coleman RE. Cardiac Phantom Evaluation of Simultaneously Acquired Dual-Isotope Rest Thallium-201/Stress Technetium-99m SPECT Images. Journal of Nuclear Medicine 34 1998-2006 (1993)

(7) Maddahi J, Rodrigues E, Berman DS, Kiat H. State-of-the-Art Myocardial Perfusion Imaging. Cardiology Clinics 12 199-222 (1994)

(8) Nakamura M, Takeda K, Ichihara T, Motomura N, Shimizu H, Saito Y, Nomura Y, Isaka N, Konishi T, Nakano T. Feasibility of Simultaneous Stress ${ }^{99 \mathrm{~m}} \mathrm{Tc}$-Sestimibi/Rest ${ }^{201} \mathrm{Tl}$ 
Dual-Isotope Myocardial Perfusion SPECT in the Detection of Coronary Artery Disease. Journal of Nuclear Medicine 40-6 895-903 (1999)

(9) Berman DS, Kiat H, Train KV, Friedman JD, Wang FP, Germano G. Dual-Isotope Myocardial Perfusion SPECT with Rest Thallium-201 and Stress Tc99m Sestimibi. Cardiology Clinics 12 261-270 (1994)

(10) Hademos GJ, Dahlbom M, Hoffman EJ. Simultaneous Dual-Isotope Technetium99m/Thallium-201 Cardiac SPET Imaging Using a Projection-Dependent Spilldown Correction Factor. European Journal of Nuclear Medicine 22 465-472 (1995)

(11) Kacperski K, Erlandsson K, Ben-Haim S, Van Gramberg D, Hutton BF. Iterative Deconvolution of Simultaneous Dual Radionuclide Projections for CdZnTe Based Cardiac SPECT. Proceedings IEEE Nuclear Science Symposium Medical Imaging Conference; p 5260-5263 (2008)

(12) Knesaurek K. A New Dual-isotope Convolution Cross-talk Correction Method: A T1201/Tc99m SPECT Cardiac Phantom Study. Medical Physics 21 1577-1583 (1994)

(13) Heart and Stroke Foundation of Canada. Heart disease - Anatomy of the Heart. http://www.heartandstroke.com/site/c.ikIQLcMWJtE/b.3532069/k.4265/Heart_disease Anatomy_of_the_Heart.htm. Reviewed August 2009. Accessed September 2012.

(14) Fuster V, Walsh RA, Harrington RA. Hurt's the Heart (Volume 1), 13 ${ }^{\text {th }}$ edition, China: McGraw-Hill Companies Inc (2011)

(15) Heart and Stroke Foundation of Canada. Heart disease -Heart Disease Conditions. http://www.heartandstroke.com/site/c.ikIQLcMWJtE/b.3483923/k.FCD0/Heart_disease_ _Heart_Disease_Conditions.htm. Reviewed August 2009. Accessed September 2012. 
(16) Cherry SR, Sorenson JA, Phelps ME. Physics in Nuclear Medicine, $3^{\text {rd }}$ Edition, New York, NY: Saunders (2003)

(17) Johns HE. Cunningham JR. The Physics of Radiology, $4^{\text {th }}$ Edition, Springfield, IL:Thomas Books (1983)

(18) Hobbie RK, Bradley JR. Intermediate Physics for Medicine and Biology, $4^{\text {th }}$ Edition, New York, NY: Springer (2007)

(19) Saha GB. Fundamentals of nuclear pharmacy, $6^{\text {th }}$ Edition, New York, London: Springer (2010)

(20) Laboratoire National Henri Becquerel: Tc99m Radionuclide Tables, http://www.nucleide.org/DDEP_WG/DDEPdata by_Z.htm. Updated January 2012. Accessed September 2012

(21) Einstein AJ, Moser KW, Thompson RC, Cerqueira MD, Henzlova, MJ. Radiation Dose to Patients from Cardiac Diagnostic Imaging. 116 1290-1305 (2007)

(22) Laboratoire National Henri Becquerel: T1201 Radionuclide Tables, http://www.nucleide.org/DDEP WG/DDEPdata by Z.htm. Updated June 2005. Accessed September 2012

(23) Bonow R, Mann DL, Zipes DP, Libby P. Braunwald's Heart Disease (Volume 2), $9^{\text {th }}$ edition, Philadelphia, PA: Saunders (2011)

(24) Shepp LA, Vardi Y. Maximum Likelihood Reconstruction for Emission Tomography. IEEE Transactions on Medical Imaging 1-2 113-122 (1982) 
(25) Blevis I, Tsukerman L, Volokh L, Hugg J, Jansen F, Bouhnik JP. CZT Gamma

Camera with Pinhole Collimator: Spectral Measurements. IEEE Nuclear Science Symposium Conference Record 4931-4932 (2008)

(26) Bocher M, Blevis IM, Tsukerman L, Shrem Y, Kovalski G, Volokh L. A Fast Cardiac Gamma Camera with Dynamic SPECT Capabilities: Design, System Validation and Future Potential. European Journal of Nuclear Medicine and Molecular Imaging 37$101887-1902(2010)$

(27) Esteves FP, Raggi P, Folks RD, Keidar Z, Wells Askew J, Rispler S, O'Connor MK, Verdes L, Garcia EV. Novel Solid-state Detector Cardiac Camera for Fast Myocardial Perfusion Imaging: Multicenter Comparison with Standard Dual Detector Cameras. Journal of Nuclear Cardiology (2009)

(28) Slomka PJ, Patton JA, Berman DS, Germano G. Advances in Technical Aspects of Myocardial Perfusion SPECT Imaging. Journal of Nuclear Cardiology 16-2 255-276 (2009)

(29) Prince JL, Links JM. Medical Imaging, Signals and Systems, $1^{\text {st }}$ edition, Upper Saddle River, NJ: Pearson Education (2006)

(30) Herzog BA, Buechel RR, Katz R, Brueckner M, Husmann L, Burger IA, Pazhenkottil AP, Valenta I, Gaemperli O, Treyer V, Kaufmann PA. Nuclear Myocardial Perfusion Imaging with a Cadmiun-Zinc-Telluride Detector Technique: Optimised Protocol for Scan Time Reduction. Journal of Nuclear Medicine 51-1 46-51 (2010)

(31) Bonow R. High-Speed Myocardial Perfusion Imaging: Dawn of a New Era in Nuclear Cardiology? Journal of the American College of Cardiology 1-2 164-166 (2008) 
(32) Buechel RR, Bernhard AH, Husmann L, Burger IA, Paxhenkottil AP, Treyer V, Valentia I, von Schulthess P, Nkoulou R, Wyss CA, Kaufmann PA. Ultrafast Nuclear Myocardial Perfusion Imaging on a New Gamma Camera with Semiconductor Detector Technique: First Clinical Validation. European Journal of Nuclear Medicine and Molecular Imaging 37 773-778 (2010)

(33) Sharir T, Slomka PJ, Berman DS. Solid-State SPECT. Technology : Fast and Furious. Journal of Nuclear Cardiology 17-5 890-896 (2010)

(34) Wernick MN, Aarsvold JN. Emission Tomography: The Fundamentals of PET and SPECT, $1^{\text {st }}$ Edition, Elsevier (2004)

(35) Leo WR. Techniques for Nuclear and Particle Physics Experiments: A How-To Approach, $2^{\text {nd }}$ Edition, Verlag, Berlin, Heidelberg, New York: Springer (1994)

(36) Sauli F. Instrumentation in High Energy Physics. Advanced Series on Directions in High Energy Physics - Vol 9, River Edge, NJ: World Scientific Publishing Co Pte. Ltd. (1993)

(37) Redus RH, Pantazis JA. Fano Factor Determination for CZT. Semiconductors for Room-Temperature Radiation Detector Applications II. Materials Research Society Symposium Proceedings 487 101-107 (1997)

(38) Volokh L, Lahat C, Binyamin E, Blevis I. Myocardial Perfusion Imaging with an Ultra-Fast Cardiac SPECT Camera - a Phantom Study. 2008 IEEE Nuclear Science Symposium Conference Record 4636-4638 (2008)

(39) Hutton BF, Buvat I, Beekman FJ. Review and Current Status of SPECT Scatter Correction. Physics in Medicine and Biology 56 R85-R112 (2011) 
(40) King MA, Tsui BMW, Pan T. Attenuation Compensation for Cardiac Single-

Photon Emission Computed Tomographic Imaging: Part 1. Impact of Attenuation and Methods of Estimating Attenuation Maps. Journal of Nuclear Cardiology 2-6 513-524 (1995)

(41) King MA, Tsui BMW, Pan T, Glick SJ, Soares EJ. Attenuation Compensation for Cardiac Single-Photon Emission Computed Tomographic Imaging: Part 2. Attenuation Compensation Algorithms. Journal of Nuclear Cardiology 3-1 55-63 (1996)

(42) Buvat I, Benali H, Todd-Pokropek A, Di Paola R. Scatter Correction in Scintigraphy: the State of the Art. European Journal of Nuclear Medicine 21-7 675-694 (1994)

(43) Frey EC, Tsui BMW, Ljungberg M. A Comparison of Scatter Compensation Methods in SPECT: Subtraction-Based Techniques Versus Iterative Reconstruction with Accurate Modelling of the Scatter Response. Record of the 1992 IEEE Nuclear Science Symposium and Medical Imaging Conference 1035-1037 (1992)

(44) Ogawa K, Harata Y, Ichihara T, Kubo A, Hashimoto S. A Practical Method for Position-Dependent Compton-Scattered Correction in Single-Photon Emission CT. IEEE Transactions on Medical Imaging 10-3 408-412 (1991)

(45) Gagnon D, Todd-Pokropek A, Arsenault A, Dupras G. Introduction to Holospectral Imaging in Nuclear Medicine for Scatter Subtraction. IEEE Transactions on Medical Imaging 8-3 245-250 (1989) 
(46) Ichihara T, Ogawa K, Motomura N, Kubo A, Hashimoto S. Compton Scatter Compensation Using the Triple-Energy Window Method for Single- and Dual-Isotope SPECT. Journal of Nuclear Medicine 34-12 2216-2221 (1993)

(47) Jaszczak RJ, Greer KL, Floyd CE, Harris CC, Coleman RE. Improved SPECT Quantification using Compensation for Scattered Photons. Journal of Nuclear Medecine 25-8 893-900 (1984)

(48) King MA, Hademenos GJ, Glick SJ. A Dual-Photopeak Window Method for Scatter Correction. Journal of Nuclear Medicine 33-4 605-612 (1992)

(49) Mas J, Hannequin P, Ben Younes R, Bellaton B, Bidet R. Scatter Correction in Planar Imaging and SPECT by Constrained Factor Analysis of Dynamic Structures (FADS). Physics in Medicine and Biology 35-11 1451-1465 (1990)

(50) Beekman FJ, den Harder JM, Viergever MA, van Rijk PP. SPECT Scatter Modeling in Non-Uniform Attenuating Objects. Physics in Medicine and Biology 42 $1133-1142(1997)$

(51) Beekman FJ, Kamphuis C, Frey EC. Scatter Compensation Methods in 3D Iterative SPECT Reconstruction: A Simulation Study. Physics in Medicine and Biology 42 1619-1632 (1997)

(52) Beekman FJ, Kamphius C and Viergever MA. Improved SPECT Quantitation Using Fully Three-Dimensional Iterative Spatially Variant Scatter Response Compensation. IEEE Transactions on Medical Imaging 15 491-499 (1996)

(53) Floyd CE, Jaszczak RJ, Coleman RE. Inverse Monte Carlo: a Unified Reconstruction Algorithm. IEEE Transactions on Nuclear Science 32 779-785 (1995) 
(54) Frey EC, Ju ZW, Tsui BMW. A Fast Projector-Backprojector Pair Modeling the Asymmetric, Spatially Varying Scatter Response Function for Scatter Compensation in SPECT Imaging. IEEE Transactions on Nuclear Science 40 1992-1997 (1993)

(55) Frey EC, Tsui BMW. A New Method for Modeling the Spatially-Variant, ObjectDependent Scatter Response Function in SPECT. 1997 IEEE Nuclear Science Symposium and Medical Imaging Conference Proceedings 1082-1086 (1996)

(56) Frey EC, Tsui BMW. A Practical Method for Incorporating Scatter in a ProjectorBackprojector for Accurate Scatter Compensation in SPECT. IEEE Transactions on Nuclear Science 40 1107-1116 (1993)

(57) Kadrmas DJ, Frey EC, Karimi SS, Tsui BMW. Fast Implementations of Iterative Reconstruction-Based Scatter Compensation in Fully 3D SPECT Image Reconstruction. Physics in Medicine and Biology 43 857-873 (1998)

(58) Vandervoot E, Cellar A, Wells RG, Blinder S, Dixon K, Pang Y. Implementation of an Analytically Based Scatter Correction in SPECT Reconstruction. IEEE Transactions on Nuclear Science 52-3 645-653 (2005)

(59) Wang X, Koral K. A Regularized Deconvolution-Fitting Method for ComptonScatter Correction in SPECT. IEEE Transactions on Medical Imaging 11-3 351-360 (1992)

(60) Axelsson B, Msaki P., Israelsson A. Subtraction of Compton-Scattered Photons in Single-Photon Emission Computerized Tomography. Journal of Nuclear Medicine 25-4 490-494 (1984) 
(61) DeJong HWAM, Beekman F. Rapid SPECT Simulation of Downscatter in NonUniform Media. Physics in Medicine and Biology 46 621-635 (2001)

(62) Beekman FJ, Eijkman EGJ, Viergever MA, Borm GF, Slipjen ETP. Object Shape Dependant PSF Model for SPECT imaging. IEEE Transactions in Nuclear Science 40 31-39(1993)

(63) Floyd CE, Jaszczak RJ, Greer KL, Coleman RE. Deconvolution of Compton Scatter in SPECT. Journal of Nuclear Medicine 26 403-408 (1985)

(64) Du Y, Frey EC. Quantitative Evaluation of Simultaneous Reconstruction with Model-Based Cross-Talk Compensation for ${ }^{99 \mathrm{~m}_{\mathrm{Tc}}}{ }^{123} \mathrm{I}$ Dual-Isotope Simultaneous Acquisition Brain SPECT. Medical Physics 36-6 2021-2033 (2009)

(65) Kangasmaa T, Kuikka J, Sohlberg A. Optimization of Simultaneous T1201/Tc99m Dual-Isotope Reconstruction with Monte-Carlo-Based Scatter Correction. International Journal of Molecular Imaging 1-9 (2012)

(66) Kadrmas DJ, Frey EC, Tsui BMW. Simultanous Technetium-99m/Thallium-201 SPECT Imaging with Model-Based Compensation for Cross-Contaminating Effects. Physics in Medicine and Biology 44 1843-1860 (1999)

(67) Wells RG, Vanderwerf K, Ruddy TD. Cross-talk Correction for Dual-Isotope Imaging with a Dedicated Cardiac SPECT Camera. Proceedings IEEE Nuclear Science Symposium Medical Imaging Conference (2010)

(68) Seibert JA. X-ray Imaging Physics for Nuclear Medicine Technologists. Part 1: Basic Principles of X-ray Production. Journal of Nuclear Medicine Technology 32-3 139$147(2004)$ 